\title{
Insect Galls of the Parque Nacional do Itatiaia (Southeast Region, Brazil)
}

\author{
VALÉRIA C. MAIA and BERNARDO MASCARENHAS \\ Departamento de Entomologia, Museu Nacional, Depto. De Entomologia, Quinta \\ da Boa Vista, São Cristóvão, 20940-040 Rio de Janeiro, RJ, Brazil \\ Manuscript received on December 20, 2016; accepted for publication on January 27, 2017
}

\begin{abstract}
The Parque Nacional do Itatiaia (PNI) (Brazilian Southeast Region) was surveyed monthly for insect galls from February/2014 to December/ 2015. A total of 432 gall morphotypes were found. This number places the PNI as the richest Atlantic forest area in number of gall morphotypes. The galls were found on 47 plant families. Among them, Asteraceae were pointed out as the superhost. The gall richness in the lower part of the PNI is higher than that of the plateau. The insect galls were found in 154 native, 56 endemic and only one exotic plant species. Concerning the conservational status, the host plants include two vulnerable species with three morphotypes together. Several new botanical records were reported. Leaves were the most galled plant organ, followed by stems. Globoid, green, glabrous and one-chambered galls were the most frequent. Cecidomyiidae were the most common gallers. Parasitoids, successors and inquilines composed the associated fauna.
\end{abstract}

Key words: Atlantic forest, Cecidomyiidae, insect-plant interactions, multitrophic relations, new records.

\section{INTRODUCTION}

The Parque Nacional do Itatiaia (PNI) is a prioritary area for the biodiversity conservation of the Atlantic forest. It is situated in the Serra da Mantiqueira on the border of the states of Rio de Janeiro, Minas Gerais, and São Paulo (Fig. 1). It has an area of 28,000 hectares, characterized by mountains and rocky hills, with altitude ranging from 540 to $2,791 \mathrm{~m}$. The flora and fauna exhibit high index of endemism. In the lowest gradients, the dense forest predominates, whereas in the highest there

Correspondence to: Valéria Cid Maia

E-mail: maiavcid@acd.ufrj.br

* Contribution to the centenary of the Brazilian Academy of Sciences. is a predominance of the altitude fields. About 1,328 plant species have been recorded in the PNI, among them, 163 are endemic. The fauna includes about 5,000 insect species, 25 reptile species, 67 amphibian species, 357 bird species and 67 mammalian species (ICMBIO 2016).

Although many inventories have been indicated a great richness of insect galls in the State of Rio de Janeiro (Maia 2013a), few gall morphotypes were recorded in the PNI (Gagné et al. 2001, Coelho et al. 2013a, Maia 2014). Furthermore, most surveys in the Atlantic forest were developed in restinga environments (Maia 2014). Other phytophysiognomies are still poorly studied, such as dense ombrophilous forest and altitude fields, both included in the PNI. In fact, only one long 
term inventory was developed in areas of dense forest (Maia et al. 2014) and altitude fields (Maia 2014). Other records of these formations are due to scattered sampling.

Several hypotheses regarding richness and distribution of gall-inducing insects were proposed by different authors (Root 1973, Fernandes and Price 1988, Lawton 1983, Sáiz and Núñez 2000, Yukawa et al. 2001, Mendonça 2001). Among them, the present work approaches the plant richness hypothesis (Southwood 1960, 1961), the harsh environment hypothesis (Fernandes and Price 1988, 1992), and the plant architecture hypothesis (Lawton 1983). The first one predicts that the richest plant taxa comprise the greatest insect gall richness, the second proposes that insect galls are especially abundant in plant species found in hot and dry habitats, and the third predicts that the most complex plants host the highest gall richness, since they offer the greatest number of niches for the insects (Lawton 1983). These three hypotheses have been supported by several authors (Fernandes and Price 1988, Fernandes and Lara 1993, Sáiz and Núñez 2000, Gonçalves-Alvim and Fernandes 2001, Yukawa et al. 2001, Lara et al. 2002, CuevasReyes et al. 2004).

The main goal of this research is to elaborate an inventory of the insect galls of the PNI and to contribute to the knowledge of their richness in two poorly studied physiognomies of the Atlantic forest.

\section{MATERIALS AND METHODS}

The PNI was investigated monthly by the authors from February/2014 to December/2015. Each campaign lasted three days performing 24 hours of field work. All campaigns together totaled 69 days with 1,656 hours of field work. All official trails were surveyed and their geographic coordinates, length, and altitude were obtained, using GPS (Table I). Each trail was investigated along all of its lenght by VCM and BM. This methodology has been adopted by several authors (Fernandes et al 1988, Urso-Guimarães et al. 2003, Santos et al. 2012).

Herbs, bushes and trees (until $2 \mathrm{~m}$ high) were examined. Leaves, buds, stems, tendrils, aerial roots, flowers, bud flowers, and fruits were investigated for galls. Preferentially fertile branches of each host plant species were removed, labeled, pressed and dried. The exsiccates were identified and deposited in the herbarium of the Universidade Federal Rural da Amazônia (UFRAM) and in the herbarium (R) of the Museu Nacional/Universidade Federal do Rio de Janeiro (UFRJ).

Galled branches were collected, packed and transported in labelled plastic bags. The galls were characterized in different morphotypes according to their shape, color, presence or absence of trichomes, and in which plant organ of occurrence. Each plastic bag contained samples of a single host plant and gall morphotype. The labels comprised the following data: trail name, date, number of the galled plant, and gall morphotype. Each host plant and gall morphotype were photographed.

In the laboratory, samples of each gall were dissected in order to observe the number of internal chamber, the food habit of the dwellers and to obtain the immature insects. Other samples were conditioned individually in labelled plastic pots to obtain the adults. Each pot was lined with a layer of toilet paper. All pots were checked daily. All obtained insects were preserved in $70 \%$ alcohol and identified by the authors. The galls were kept in the pots until the adults'emergence or until they began to putrefy.

The Cecidomyiidae specimens were later mounted on microscope slides, following the methodology of Gagné (1994), and identified in genus based on the keys of Gagné (op. cit.). The insects were deposited in the Entomological Collection of Museu Nacional (MNRJ). 
TABLE I

\begin{tabular}{|c|c|c|c|}
\hline Trail & \multicolumn{2}{|c|}{ Geographic coordinate } & \multirow[t]{2}{*}{ Altitude } \\
\hline & trailhead & trail end & \\
\hline \multirow{2}{*}{ BR-485 (Headquarter - Maromba) (RJ) } & $\mathrm{S} 22^{\circ} 27^{\prime} 54^{\prime \prime}$ & $22^{\circ} 25^{\prime} 49^{\prime \prime}$ & $837 \mathrm{~m}$ \\
\hline & WO $44^{\circ} 35^{\prime} 28^{\prime \prime}$ & $44^{\circ} 37^{\prime} 16^{\prime \prime}$ & $840 \mathrm{~m}$ \\
\hline \multirow{2}{*}{ Cachoeira Itaporani (RJ) } & $\mathrm{S} 22^{\circ} 25^{\prime} 42^{\prime}$ & $22^{\circ} 25^{\prime} 40^{\prime \prime}$ & \\
\hline & WO443' $10^{\prime \prime}$ & $44^{\circ} 37^{\prime} 20^{\prime \prime}$ & $1,091 \mathrm{~m} \mathrm{I,15/m}$ \\
\hline \multirow{2}{*}{ Cachoeira Véu da Noiva (RJ) } & $\mathrm{S} 22^{\circ} 25^{\prime} 43^{\prime \prime}$ & $22^{\circ} 25^{\prime} 40^{\prime \prime}$ & $1,124 \mathrm{~m}$ \\
\hline & WO $44^{\circ} 37^{\prime} 11^{\prime \prime}$ & $44^{\circ} 37^{\prime} 12^{\prime \prime}$ & $1,154 \mathrm{~m}$ \\
\hline \multirow[t]{2}{*}{ Lago Azul (RJ) } & $\mathrm{S} 22^{\circ} 26^{\prime} 58^{\prime \prime}$ & $22^{\circ} 27^{\prime} 01^{\prime \prime}$ & $1,041 \mathrm{~m}$ \\
\hline & $\begin{array}{l}\text { WO } 44^{\circ} 36^{\prime} 37^{\prime \prime} \\
\text { S22 } 25^{\circ} 44^{\prime \prime}\end{array}$ & $\begin{array}{l}44^{\circ} 36^{\prime} 52^{\prime \prime} \\
22^{\circ} 27^{\prime} 01^{\prime \prime}\end{array}$ & $\begin{array}{l}879 m \\
739 m\end{array}$ \\
\hline Ecoarte-Lago Azul (RJ) & WO $44^{\circ} 37^{\prime} 11^{\prime \prime}$ & $44^{\circ} 36^{\prime} 52^{\prime \prime}$ & $879 \mathrm{~m}$ \\
\hline \multirow{2}{*}{ Cachoeira Poranga (RJ) } & $\mathrm{S} 22^{\circ} 27^{\prime} 40^{\prime \prime}$ & $22^{\circ} 26^{\prime} 32^{\prime \prime}$ & $947 \mathrm{~m}$ \\
\hline & WO443' $15^{\prime \prime}$ & $44^{\circ} 36^{\prime} 44^{\prime \prime}$ & $826 \mathrm{~m}$ \\
\hline Três Picos (incluing Cachoeira Bela Vista) (RJ) & $\mathrm{S} 22^{\circ} 25^{\prime} 26^{\prime \prime} \mathrm{WO} 44^{\circ} 35^{\prime} 31^{\prime \prime}$ & $\begin{array}{l}22^{\circ} 25^{\prime} 31^{\prime \prime} \\
44^{\circ} 35^{\prime} 01^{\prime \prime}\end{array}$ & $\begin{array}{l}1,541 \mathrm{~m} \\
1,596 \mathrm{~m}\end{array}$ \\
\hline Centro de Visitantes (RJ) & \multicolumn{2}{|l|}{$\mathrm{S} 22^{\circ} 27^{\prime} 04^{\prime \prime}$} & $877 \mathrm{~m}$ \\
\hline Casa 16 (RJ) & $\mathrm{S} 22^{\circ} 27^{\prime} 16^{\prime \prime}$ & $22^{\circ} 27^{\prime} 16^{\prime \prime}$ & $841 \mathrm{~m}$ \\
\hline \multirow{2}{*}{$\begin{array}{l}\text { Hotel Donati } \\
\text { (entrada-represa) (RJ) }\end{array}$} & \multirow{2}{*}{$\mathrm{S} 22^{\circ} 26^{\prime} 43^{\prime \prime} \mathrm{WO} 44^{\circ} 36^{\prime} 04^{\prime \prime}$} & $\begin{array}{l}44^{\circ} 36^{\prime} 20^{\prime \prime} \\
22^{\circ} 26^{\prime} 36^{\prime \prime}\end{array}$ & $\begin{array}{l}853 \mathrm{~m} \\
935 \mathrm{~m}\end{array}$ \\
\hline & & $\begin{array}{l}44^{\circ} 35^{\prime}, 54^{\prime \prime} \\
22^{\circ} 26^{\prime} 19^{\prime \prime}\end{array}$ & $\begin{array}{l}956 \mathrm{~m} \\
935 \mathrm{~m}\end{array}$ \\
\hline Hotel Donati-Simon 1 (RJ) & $\mathrm{S} 22^{\circ} 26^{\prime} 43^{\prime \prime} \mathrm{WO} 44^{\circ} 6^{\prime} 04^{\prime \prime}$ & $\begin{array}{l}44^{\circ} 36^{\prime} 29^{\prime \prime} \\
22^{\circ} 26^{\prime} 22^{\prime \prime}\end{array}$ & $1,111 \mathrm{~m}$ \\
\hline Donati-Simon 2 (RJ) & $\mathrm{S} 22^{\circ} 26^{\prime} 19^{\prime \prime} \mathrm{WO} 44^{\circ} 36^{\prime} 29^{\prime \prime}$ & $\begin{array}{l}44^{\circ} 35^{\prime} 47^{\prime \prime} \\
22^{\circ} 26^{\prime} 46^{\prime \prime}\end{array}$ & $\begin{array}{l}927 \mathrm{~m} \\
948 \mathrm{~m}\end{array}$ \\
\hline Viúva Hansen (RJ) & $\begin{array}{l}\text { WO } 44^{\circ} 35^{\prime} 55^{\prime \prime} \\
\text { S22 } 27^{\circ} 19.0^{\prime \prime}\end{array}$ & $\begin{array}{l}44^{\circ} 36^{\prime} 05^{\prime \prime} \\
22^{\circ} 27^{\prime} 40^{\prime \prime}\end{array}$ & $\begin{array}{l}953 \mathrm{~m} \\
783 \mathrm{~m}\end{array}$ \\
\hline Barbosa Rodrigues-Mirante do Último Adeus (RJ) & WO443'ㄱ' & $44^{\circ} 36^{\prime} 15^{\prime \prime}$ & $765 \mathrm{~m}$ \\
\hline \multirow{2}{*}{ Barbosa Rodrigues (obelisk) (RJ) } & $\mathrm{S} 22^{\circ} 27^{\prime} 17^{\prime \prime}$ & $22^{\circ} 27^{\prime} 09^{\prime \prime}$ & $736 \mathrm{~m}$ \\
\hline & WO443' $37^{\prime \prime}$ & $44^{\circ} 36^{\prime} 36^{\prime \prime}$ & $882 \mathrm{~m}$ \\
\hline \multirow{2}{*}{ Cachoeira do Pitu (RJ) } & $\mathrm{S} 22^{\circ} 26^{\prime} 33^{\prime \prime}$ & $22^{\circ} 26^{\prime} 14^{\prime \prime}$ & $944 \mathrm{~m}$ \\
\hline & WO44³6'44”' & $44^{\circ} 36^{\prime} 49^{\prime \prime}$ & $902 \mathrm{~m}$ \\
\hline \multirow{2}{*}{ Casa 33 (RJ) } & $\mathrm{S} 22^{\circ} 24^{\prime} 01^{\prime \prime}$ & $22^{\circ} 24^{\prime} 01^{\prime \prime}$ & $1,062 \mathrm{~m}$ \\
\hline & WO443'ㄹ" & $44^{\circ} 39^{\prime} 12^{\prime \prime}$ & $1,062 \mathrm{~m}$ \\
\hline Casa 25 (RJ) & $\mathrm{S} 22^{\circ} 27^{\prime} 18^{\prime \prime} \mathrm{WO} 44^{\circ} 36^{\prime} 37^{\prime \prime}$ & $22^{\circ} 26^{\prime} 42^{\prime \prime}$ & $762 \mathrm{~m}$ \\
\hline Pedra do Camelo (RJ) & $\begin{array}{c}\mathrm{S} 22^{\circ} 26^{\prime} 17^{\prime \prime} \\
\mathrm{WO} 44^{\circ} 37^{\prime} 31^{\prime \prime}\end{array}$ & $44^{\circ} 36^{\prime} 50^{\prime \prime}$ & $\begin{array}{r}813 \mathrm{~m} \\
2,194 \mathrm{~m}\end{array}$ \\
\hline Casa de Pedra (RJ) & $\mathrm{S} 22^{\circ} 22^{\prime} 07^{\prime \prime} \mathrm{WO} 44^{\circ} 42^{\prime} 43^{\prime \prime}$ & & $2,010 \mathrm{~m}$ \\
\hline \multirow{2}{*}{ Agulhas Negras (RJ) } & $\mathrm{S} 22^{\circ} 22^{\prime} 52^{\prime \prime}$ & $22^{\circ} 23^{\prime} 06^{\prime \prime}$ & $1,041 \mathrm{~m}$ \\
\hline & WO $44^{\circ} 41^{\prime} 08^{\prime \prime}$ & $44^{\circ} 40^{\prime} 21^{\prime \prime}$ & $898 \mathrm{~m}$ \\
\hline \multirow{2}{*}{ Cachoeira do Aiuruoca (MG) } & $\mathrm{S} 22^{\circ} 23^{\prime} 06^{\prime \prime}$ & $22^{\circ} 21^{\prime} 08^{\prime \prime}$ & $898 \mathrm{~m}$ \\
\hline & WO44²4’21”' & $44^{\circ} 40^{\prime} 07^{\prime \prime}$ & $2,382 \mathrm{~m}$ \\
\hline Posto Marcão-Abrigo Rebouças (RJ/MG) & $\begin{array}{l}\mathrm{S} 22^{\circ} 23^{\prime} 02^{\prime \prime} \\
\text { WO4 } 44^{\circ} 40^{\prime} 06^{\prime \prime}\end{array}$ & $\begin{array}{l}22^{\circ} 22^{\prime} 27^{\prime \prime} \\
44^{\circ} 42^{\prime} 04^{\prime \prime}\end{array}$ & $2,290 \mathrm{~m} \mathrm{2,469m}$ \\
\hline & $\mathrm{S} 22^{\circ} 22^{\prime} 37^{\prime \prime}$ & $22^{\circ} 22^{\prime} 27^{\prime \prime}$ & $2,501 \mathrm{~m}$ \\
\hline Pedra do Altar (RJ/MG) & $\begin{array}{l}\text { WO } 44^{\circ} 42^{\prime} 10^{\prime \prime} \\
\text { S2 } 22^{\circ} 23^{\prime} 01^{\prime \prime}\end{array}$ & $\begin{array}{l}44^{\circ} 40^{\prime} 27^{\prime \prime} \\
22^{\circ} 22^{\prime} 33^{\prime \prime}\end{array}$ & $\begin{array}{l}2,585 \mathrm{~m} \\
2,340 \mathrm{~m}\end{array}$ \\
\hline Asa de Hermes & WO $44^{\circ} 40^{\prime} 06^{\prime \prime}$ & $44^{\circ} 39^{\prime} 53^{\prime \prime}$ & $2,870 \mathrm{~m}$ \\
\hline
\end{tabular}


TABLE I (continuation)

\begin{tabular}{|c|c|c|c|}
\hline Trail & \multicolumn{2}{|c|}{ Geographic coordinate } & Altitude \\
\hline \multirow{2}{*}{ Pedra do Sino } & $\mathrm{S} 22^{\circ} 21^{\prime} 55^{\prime \prime}$ & $22^{\circ} 22^{\prime} 13^{\prime \prime}$ & $2,406 \mathrm{~m}$ \\
\hline & 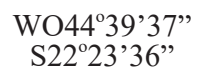 & $\begin{array}{l}44^{\circ} 39^{\prime} 45^{\prime \prime} \\
22^{\circ} 23^{\prime} 56^{\prime \prime}\end{array}$ & $\begin{array}{l}2,591 \mathrm{~m} \\
2,372 \mathrm{~m}\end{array}$ \\
\hline Prateleiras (RJ) & $\begin{array}{l}\text { WO } 44^{\circ} 40^{\prime} 22^{\prime \prime} \\
\mathrm{S} 22^{\circ} 25^{\prime} 49^{\prime \prime}\end{array}$ & $\begin{array}{l}44^{\circ} 40^{\prime} 16^{\prime \prime} \\
22^{\circ} 22^{\prime} 27^{\prime \prime}\end{array}$ & $\begin{array}{c}2,453 \mathrm{~m} \\
840 \mathrm{~m}\end{array}$ \\
\hline Travessia Ruy Braga (RJ) & $\begin{array}{l}\text { WO } 44^{\circ} 37^{\prime} 16^{\prime \prime} \\
\text { S22 } 26^{\prime} 02^{\prime \prime}\end{array}$ & $\begin{array}{l}44^{\circ} 42^{\prime} 04^{\prime \prime} \\
22^{\circ} 25^{\prime} 15^{\prime \prime}\end{array}$ & $\begin{array}{l}2,469 \mathrm{~m} \\
1,727 \mathrm{~m}\end{array}$ \\
\hline Água Branca-Ruy Braga (RJ) & $\begin{array}{l}\text { WO } 44^{\circ} 38^{\prime} 15^{\prime \prime} \\
\text { S22 } 21^{\prime} 44^{\prime \prime}\end{array}$ & $\begin{array}{l}44^{\circ} 38^{\prime} 26^{\prime \prime} \\
22^{\circ} 19^{\prime} 14^{\prime \prime}\end{array}$ & $\begin{array}{l}1,798 \mathrm{~m} \\
2,381 \mathrm{~m}\end{array}$ \\
\hline Travessia Serra Negra (RJ/MG) & $\begin{array}{l}\text { WO } 44^{\circ} 40^{\prime} 09^{\prime \prime} \\
\text { S22 } 22^{\prime} 27^{\prime \prime}\end{array}$ & $44^{\circ} 36^{\prime} 43^{\prime \prime}$ & $1,524 \mathrm{~m}$ \\
\hline Morro do Couto (RJ) & $\begin{array}{l}\mathrm{WO} 44^{\circ} 42^{\prime} 05^{\prime \prime} \\
\mathrm{S} 22^{\circ} 23^{\prime} 51^{\prime \prime}\end{array}$ & $22^{\circ} 23^{\prime} 55^{\prime \prime}$ & $\begin{array}{l}2,449 \mathrm{~m} \\
2,380 \mathrm{~m}\end{array}$ \\
\hline Pedra da Maçã, da Tartaruga and Assentada (RJ) & $\begin{array}{l}\mathrm{WO} 44^{\circ} 40^{\prime} 15^{\prime \prime} \\
\mathrm{S} 22^{\circ} 22^{\prime} 23^{\prime \prime}\end{array}$ & $\begin{array}{l}44^{\circ} 39^{\prime} 58^{\prime \prime} \\
22^{\circ} 22^{\prime} 23^{\prime \prime}\end{array}$ & $\begin{array}{l}2,384 \mathrm{~m} \\
2,344 \mathrm{~m}\end{array}$ \\
\hline Cinco Lagos (RJ) & $\begin{array}{l}\text { WO } 44^{\circ} 42^{\prime} 11^{\prime \prime} \\
\text { S22 } 22^{\prime} 23^{\prime \prime}\end{array}$ & $\begin{array}{l}44^{\circ} 40^{\prime} 43^{\prime \prime} \\
22^{\circ} 21^{\prime} 52^{\prime \prime}\end{array}$ & $\begin{array}{l}2,533 \mathrm{~m} \\
2,248 \mathrm{~m}\end{array}$ \\
\hline Ovos da Galinha (MG) & WO $44^{\circ} 42^{\prime} 11^{\prime \prime}$ & $44^{\circ} 39^{\prime} 34^{\prime \prime}$ & $2,430 \mathrm{~m}$ \\
\hline
\end{tabular}

The botanic names and authors were checked in Flora do Brasil (2016), as well as the plant species distribution in Brazil, their conservational status (NE - not evaluated, LC - less concerning, VU vulnerable), and classification as native, endemic or exotic. The biomes of occurrence of all endemic species were verified in the same site.

\section{RESULTS AND DISCUSSION}

A total of 432 gall morphotypes were found in the Parque Nacional do Itatiaia. Other gall morphotypes were recorded in the PNI: 19 by Coelho et al. 2013a, 38 by Maia 2014, both in the altitude fields, and two by Gagné et al. 2001 (no data on phytophysiognomy). Among them, only four were found in the present study. Adding our results with the previous ones, the PNI totalizes 487 gall morphotypes. This number places the PNI as the richest area of the Atlantic forest in number of insect galls, followed by Vale do Rio Doce (Minas Gerais), Santa Teresa (Espírito Santo) and Bertioga (São Paulo) (Table II). Comparing the gall richness of the PNI with data on other Brazilian biomes, we notice that the PNI remains in the first place (Table III).

But, these inventories (mentioned in tables II and III) have adopted different sampling methods, resulting in different sampling efforts. Furthermore, as none of them presented a rarefaction curve, one cannot conclude if the surveyed areas were well sampled. Several authors argue that insect galls are especially abundant in plant species found in hot and dry habitats (Fernandes and Price 1988, 1992, Lara and Fernandes 1996, Price et al. 1998), but many recent Brazilian inventories do not confirm that initial hypothesis (such as the present one), since a greater number of insect galls have been recorded in Amazonian and Atlantic forests (wet habitats) than in Cerrado and Caatinga (dry habitats).

In the present inventory, galls were found in 47 plant families. Among them, Asteraceae hosted the greatest gall richness (93 morphotypes), followed by Melastomataceae (with 66 morphotypes). All other families hosted less than 30 gall morphotypes. Five of them hosted from 29 to 20 gall morphotypes: Fabaceae (29), Myrtaceae (26), Solanaceae (23), 
Piperaceae (20), and Sapindaceae (20); three from 16 to 10: Rubiaceae (16), Euphorbiaceae (14) and Apocynaceae (10). The great majority (76.5\%) comprised less than ten morphotypes (Table IV). Two other host families, not found in the present study, were recorded by Maia 2014 in the PNI: Campanulaceae and Iracinaceae. Adding these two families to our data, the number of host plant families in the PNI reaches 49.

The host plants included 130 determined genera and 145 determined species. Seven plants were identified at family level due to the lack of fertile samples. Eight other host plants, identified only at genus, were previously recorded in the PNI by Maia 2014: Centropogon sp. (Campanulaceae), Humiriantheca sp. (Icacinaceae), Hyptis sp. (Lamiaceae), Licania (Lauraceae), Mouriri sp. (Melastomataceae), Marlierea sp. (Myrtaceae), Homalium sp. and Xylosma sp. (Salicacae), but we did not find these genera in our survey. Six other host plant species were previously recorded in the PNI by Coelho et al. 2013a: Baccharis brevifolia DC., B. dubia Deble and A.S. de Oliveira, $B$. itatiaiae Wawra, B. pseudomyriocephala Malag., B. uncinella DC. (Asteraceae), and Croton dichrous Müll. Arg. (Euphorbiaceae), (Coelho et al. 2013a), but they were not found by us. Adding the values, the PNI totalizes 151 galled plant species.

Although part of the host plants were identified until family and genus level, we believe that the number of gall morphotypes was satisfactorily estimate, as the comparison among the dried plant specimens allowed to segregate the different morphospecies.

Orchidaceae, Fabaceae, Asteraceae, Bromeliaceae, Poaceae, Myrtaceae, Melastomataceae, Euphorbiaceae, Rubiaceae, and Apocynaceae are the ten most speciose Angiospermae families of the Atlantic forest (Jardim Botânico do Rio de Janeiro 2016). These data confirm the plant richness hypothesis, which predicts that the richest plant taxa comprise the greatest insect gall richness
(Southwood 1960, 1961). Although Orchidaceae, Bromeliaceae and Poaceae are among the most diverse plant families, they host few galls throughout the world, which can be explained by the hypothesis of the plant architecture. According to this hypothesis the most complex plants host the highest gall richness, since they offer the greatest number of niches for the insects (Lawton 1983). The architecture of Orchidaceae, Bromeliaceae and Poaceae are less complex in comparison to that of the other superhost families. So, these two hypotheses together explain our results.

The gall richness in the lower part of the PNI was higher than that of the plateau: 183 gall morphotypes were restricted to the former, while only 26 were restricted to the later (Table V). This result was already expected as the lower part comprises a greater diversity of flora as well as plants with higher architectural complexity than those of the plateau. Galls found in the Travessia Ruy Braga and Travessia Serra Negra were not considered as these crossings extend from the lower part of the PNI to the plateau.

The insect galls were found in 154 native and one exotic plant species. The former hosted 341 gall morphotypes and the latter only one. Among the native plants, 56 are endemic in Brazil and they hosted 88 gall morphotypes. Among the endemic plant species, 27 have been recorded exclusively in the Atlantic forest and they hosted 39 gall morphotypes. Adding to the previous data, the number of native hosts rises to 167 (seven in Coelho et al. 2013a and six in Maia 2014) and the number of endemic plants to 63 (all in Coelho et al. 2013a). The native plants recorded by these authors presented 15 and nine gall morphotypes, respectively, totalizing 365 , while the endemic plants with 15 gall morphotypes recorded by Coelho et al. 2013a totalizing 103. As the galling insects are specific-species (Carneiro et al. 2009a, Joy and Crespi 2007, Price 2005, Yukawa and Rohfritsch 
TABLE II

\begin{tabular}{lcc}
\hline \multicolumn{1}{c}{ Locality } & $\begin{array}{c}\text { Nr. of insect } \\
\text { galls }\end{array}$ & Reference \\
\hline $\begin{array}{l}\text { Parque Nacional do } \\
\text { Itatiaia } \\
\text { (Southeastern }\end{array}$ & 487 & $\begin{array}{c}\text { Present } \\
\text { inventory }+ \\
\text { Coelho et al. }\end{array}$ \\
Brazil: RJ, MG) & & $\begin{array}{c}2013 \mathrm{a}+\text { Maia } \\
\text { 2014 + Gagné } \\
\text { et al. 2001 }\end{array}$ \\
$\begin{array}{l}\text { Vale do Rio Doce } \\
\text { (Southeastern }\end{array}$ & & $\begin{array}{c}\text { Fernandes et al. } \\
\text { Brazil: MG) }\end{array}$ \\
$\begin{array}{l}\text { Santa Teresa } \\
\text { (Southeastern }\end{array}$ & 2001 \\
$\begin{array}{l}\text { Brazil: ES) } \\
\text { Bertioga } \\
\text { (Southeastern }\end{array}$ & 265 & Maia et al. 2014 \\
Brazil: SP) & & \\
\hline
\end{tabular}

TABLE III

\begin{tabular}{|c|c|c|c|}
\hline Biome & Locality & $\begin{array}{l}\text { Nr. of } \\
\text { insect } \\
\text { galls }\end{array}$ & Reference \\
\hline $\begin{array}{l}\text { Atlantic } \\
\text { forest }\end{array}$ & $\begin{array}{c}\text { Parque } \\
\text { Nacional do } \\
\text { Itatiaia } \\
\text { (Southeastern } \\
\text { Brazil: MG, } \\
\text { RJ) }\end{array}$ & 487 & $\begin{array}{c}\text { Present } \\
\text { inventory }+ \\
\text { Coelho et } \\
\text { al. 2013a }+ \\
\text { Maia 2014 + } \\
\text { Gagné et al. } \\
2001\end{array}$ \\
\hline $\begin{array}{l}\text { Amazonian } \\
\text { forest }\end{array}$ & $\begin{array}{l}\text { Porto } \\
\text { Trombetas } \\
\text { (Northern } \\
\text { Brazil; AM) } \\
\text { Cadeia do }\end{array}$ & 309 & $\begin{array}{c}\text { Almada and } \\
\text { Fernandes } \\
2011\end{array}$ \\
\hline Cerrado & $\begin{array}{c}\text { Espinhaço } \\
\text { (Southeastern } \\
\text { Brazil: MG) } \\
\text { Corumbá }\end{array}$ & 241 & $\begin{array}{c}\text { Carneiro et } \\
\text { al. } 2009\end{array}$ \\
\hline Pantanal & $\begin{array}{l}\text { (Midwestern } \\
\text { Brazil: MS } \\
\text { Several }\end{array}$ & 133 & $\begin{array}{c}\text { Julião et al. } \\
2002\end{array}$ \\
\hline Caatinga & $\begin{array}{c}\text { municipalities } \\
\text { (Northeastern } \\
\text { Brazil: PE) }\end{array}$ & 64 & $\begin{array}{c}\text { Santos et al. } \\
2011 \mathrm{a}\end{array}$ \\
\hline Pampa & & no data & \\
\hline
\end{tabular}

TABLE IV

\begin{tabular}{lc}
\hline \multicolumn{1}{c}{ Family $(\mathbf{n = 4 7})$} & Number of gall morphotypes \\
\hline Acanthaceae & $05(1.1 \%)$ \\
Anacardiaceae & $03(<1.0 \%)$ \\
Annonaceae & $03(<1.0 \%)$ \\
Apocynaceae & $10(2.3 \%)$ \\
Aquifoliaceae & $02(<1.0 \%)$ \\
Araceae & $03(<1.0 \%)$ \\
Asteraceae & $\mathbf{9 3}(\mathbf{2 1 . 5 \% )}$
\end{tabular}

TABLE IV (continuation)

\begin{tabular}{|c|c|}
\hline Family $(n=47)$ & Number of gall morphotypes \\
\hline Berberidaceae & $01(<1.0 \%)$ \\
\hline Bignoniaceae & $08(1.8 \%)$ \\
\hline Boraginaceae & $01(<1.0 \%)$ \\
\hline Burseraceae & $04(<1.0 \%)$ \\
\hline Cannabaceae & $03(<1.0 \%)$ \\
\hline Combretaceae & $02(<1.0 \%)$ \\
\hline Convolvulaceae & $05(1.1 \%)$ \\
\hline Cunnoniaceae & $02(<1.0 \%)$ \\
\hline Curcubitaceae & $01(<1.0 \%)$ \\
\hline Dilleniaceae & $04(<1.0 \%)$ \\
\hline Dioscoriaceae & $03(<1.0 \%)$ \\
\hline Ebenaceae & $01(<1.0 \%)$ \\
\hline Euphorbiaceae & $14(3.2 \%)$ \\
\hline Fabaceae & $29(6.7 \%)$ \\
\hline Lamiaceae & $06(1.4 \%)$ \\
\hline Lauraceae & $05(1.1 \%)$ \\
\hline Loranthaceae & $09(2.1 \%)$ \\
\hline Malpighiaceae & $03(<1.0 \%)$ \\
\hline Melastomataceae & $66(15.8 \%)$ \\
\hline Meliaceae & $04(<1.0 \%)$ \\
\hline Menispermaceae & $01(<1.0 \%)$ \\
\hline Monimiaceae & $01(<1.0 \%)$ \\
\hline Moraceae & $01(<1.0 \%)$ \\
\hline Myrtaceae & $26(6.0 \%)$ \\
\hline Nyctaginaceae & $09(2.1 \%)$ \\
\hline Onagraceae & $01(<1.0 \%)$ \\
\hline Orchidaceae & $01(<1.0 \%)$ \\
\hline Piperaceae & $20(4.6 \%)$ \\
\hline Polypodiaceae & $01(<1.0 \%)$ \\
\hline Primulaceae & $09(2.1 \%)$ \\
\hline Proteaceae & $03(<1.0 \%)$ \\
\hline Pteridaceae & $01(<1.0 \%)$ \\
\hline Rosaceae & $02(<1.0 \%)$ \\
\hline Rubiaceae & $16(3.7 \%)$ \\
\hline Salicaceae & $03(<1.0 \%)$ \\
\hline Santalaceae & $02(<1.0 \%)$ \\
\hline Sapindaceae & $20(4.6 \%)$ \\
\hline Solanaceae & $23(5.3 \%)$ \\
\hline Verbenaceae & $01(<1.0 \%)$ \\
\hline Vitaceae & $01(<1.0 \%)$ \\
\hline Total & 432 \\
\hline
\end{tabular}

TABLE V

\begin{tabular}{lc}
\hline $\begin{array}{c}\text { Areas of the Parque } \\
\text { Nacional do Itatiaia }\end{array}$ & Nr. gall morphotypes \\
\hline Only in the lower part & 183 \\
Only in the plateau & 26 \\
In both areas & 02 \\
\hline
\end{tabular}


2005), we suggest the endemism of those gallers which are associated with endemic plants.

Concerning the conservational status, the host plants include two vulnerable species: Chionolaena lychnophorioides Sch. Bip. (Asteraceae) with one gall morphotype and Eugenia bunchosiifolia Nied. (Myrtaceae) with two gall morphotypes. The other plant species have not yet been evaluated. Considering again the high specifity of the galling insects, we suggest that the gallers responsible for the induction of these three morphotypes on vulnerable hosts are also vulnerable.

Several new botanical records were reported: 57 plant species and four plant genera were recorded for the first time hosting insect galls, one genera and 24 species were recorded for the first time in the Atlantic forest (being one genus and two species in ombrophilous forest and six species in altitude fields), one genus and 16 species in the Southeast Region of Brazil, one genus and 18 species in the State of Rio de Janeiro, and two species in the State of Minas Gerais.

The galls were found in leaves, stems, buds, flower buds, aerial roots and tendril. No fruit galls were observed. The leaves were the most galled plant organ, followed by stems and buds (Table VI). The highest frequency of galls on leaves is a world pattern, as already pointed out by Felt (1940). In Brazil, all published gall inventories confirm it. Leaves represent a frequent and abundant resource, especially in vegetal formations where there is no seasonal leaf loss. These features facilitate their use by herbivores.

Several distinct gall shapes were found, but the globoid and fusiform shapes were the most frequent, with $42.03 \%$ and $35.10 \%$, respectively (Table VII). The fusiform shape occurred mainly in the stem, leaf petiole and leaf midvein, whereas globoid shape was in the blade leaf. These shapes have been indicated in other Brazilian inventories as the most commom (Carvalho-Fernandes et al.
2016), as well as in other areas of the Neotropical region (Isaias et al. 2013).

Green, brown, yellow, red, purple, orange and whitish galls were observed. The most common colors were green and brown (Table VIII), the same colors of most of the galled plant organs. Some morphotypes showed color variation: green/red, green/brown, green/yellow, yellow/red, green/red/ brown, and reddish/green/yellow. This variation can be related to the gall and/or the host plant organ maturity, as well as to the sun exposure, oxidation and others (Fernandes et al. 2012, Maia et al. 2014).

Most morphotypes were glabrous (72.35\%), while $27.65 \%$ exhibited trichomes or other indumentum. The majority of the galls were one-chambered (87.02\%), whereas $9.39 \%$ were multichambered, and $3.59 \%$ showed a variable number of internal chambers (from one to several). The predominance of glabrous and one-chambered galls has been indicated in all Brazilian inventories. Generally, a single galling larva is found in onechambered galls. We think that this can represent a strategy against predators and parasitoids: if the gall is attacked, only one individual dies, whereas in multichambered galls, several individuals die.

The galling guild was composed by Diptera (Cecidomyiidae and Tephritidae), Lepidoptera, Coleoptera, Hemiptera, Thysanoptera, and Hymenoptera (Table IX). Cecidomyiidae were the most frequent inducers, with about $65 \%$ of the total of the determined gallers, followed by Lepidoptera (with about 20\%) and Hemiptera (with only about 7\%). Coleoptera, Hymenoptera and Hemiptera were responsible together for less than $4 \%$ of the gall morphotypes. Cecidomyiidae are the most frequent gallers throughout the world (Felt 1940). Several gallers were not determined, as their galls had already been collected empty or occupied by natural enemies or by several different insects, which could be inducers or inquilines according to the literature. 
The majority of the Dipteran and Hemipteran galls were found in the lower part of the PNI. Differing from them, the majority of the Lepidopteran galls occurred in the plateau. As few Coleopteran, Hymenopteran and Thysanopteran galls were found, we can not verify this. Cecidomyiidae (Diptera) and Hemiptera induced galls mainly on leaves, whereas Lepidoptera mainly in buds. Houard (1933) had already pointed this pattern out for the first two insect groups and Maia (2006) for Lepidoptera. Coleoptera, Hymenoptera and Thysanoptera were not evaluated, due to the low number of their galls.

The associated fauna comprised parasitoids, successors and inquilines (Table X). The first, represented exclusively by Hymenoptera, were the most frequent guild, found on 99 gall morphotypes. The successors included Thysanoptera (on two gall morphotypes), Formicidae (also on two) and Psocoptera (on a single gall morphotype). As inquilines, Diptera, Lepidoptera, Coleoptera, Thysanoptera, and Hemiptera were obtained from 30 gall morphotypes. Among them, Diptera were the most common. They were represented by three taxa: Cecidomyiidae on four morphotypes, Muscomorpha also on four and Sciaridae on two, totalizing 10 gall morphotypes. Two genera of Cecidomyiidae were identified: Contarinia Rondani, 1860 and Clinodiplosis Kieffer, 1894. Lepidoptera were the second most common inquilines, with occurrence on seven morphotypes, followed by Coleoptera, Thysanoptera and Hemiptera, obtained from six, five and four gall morphotypes, respectively.

Two morphotypes hosted different inquilines: Hemiptera (Aphidae) + Diptera (Muscomorpha) and Diptera (Cecidomyiidae: Contarinia sp. + Clinodiplosis sp.) + Coleoptera. Twelve morphotypes comprised different guilds, among them nine hosted parasitoids and inquilines (on Fischeria sp.1, Mikania glomerata Spreng., Symphyopappus reticulatus Baker, Croton floribundus Spreng., Inga grandiflora Ducke, Clidemia sp.1, Neea oppositifolia Ruiz and Pav., Piper turbeculatum Jacq., and Serjania glutinosa Radlk.), two hosted parasitoids, inquilines and successors (on Fischeria sp.1 and Mikania glomerata Spreng), and a single morphotype hosted inquilines and successors (on Baccharis reticularia DC). All mentioned insect taxa have been already recorded as associated fauna in other Brazilian inventories, as well as multitrophic relations.

Among the family Cecidomyiidae, gallers and inquilines are comprised 11 genera. The most diverse were Clinodiplosis (with eight species) and Contarinia (with five) (Table XI). Several species of gall midges were not determined due to the lack of material: male, female, larva, and pupa are necessary for their identification.

Nine species of gall midges were identified in the present work: Asphondylia glomeratae Gagné, 2001; A. moehni Skuhravá, 1989; Alycaulus globulus Gagné, 2001; Liodiplosis conica Gagné, 2001; L. cylindrica Gagné, 2001; L. spherica Gagné, 2001; Neolasioptera eugeniae Maia, 1993; Perasphondylia mikaniae Gagné, 2001; and Schismatodiplosis lantanae Rübsaamen 1916. Among them, only two have been previously recorded in the PNI, Alycaulus globulus and Perasphondylia mikaniae (Gagné et al. 2001), the others are recorded for the first time.

Besides, eigth galling genera, Brugmmania, Clinodiplosis, Contarinia, Dasineura, Lopesia, Neolasioptera, Schismatodiplosis and Zalepidota were also registered in the PNI for the first time.

Several new interactions between the Dipteran gallers and the host plants were reported: the family Cecidomyiidae is associated with 35 plant species (distributed in 12 botanical families) and six plant genera for the first time. Futhermore, the family Tephritidae is associated with galls on Vernonanthura H. Rob. (Asteraceae) and the infraorder Muscomorpha with galls on Dolichandra unguis-cati (L.) L. G.Lohmann (Bignoniaceae) and 
TABLE VI

\begin{tabular}{|c|c|}
\hline Galled plant organ & Number of gall morphotypes $(n=432)$ \\
\hline Leaf & $221(47.96 \%)$ \\
\hline Stem & $124(28.06 \%)$ \\
\hline Bud & $99(22.40 \%)$ \\
\hline Tendril & $03(0.68 \%)$ \\
\hline Root & $03(0.68 \%)$ \\
\hline Flower bud & $1(0.22 \%)$ \\
\hline Fruit & $0(0.0 \%)$ \\
\hline \multicolumn{2}{|c|}{ TABLE VII } \\
\hline Gall shape & Nr. of gall morphotypes \\
\hline Globoid & $182(42.03 \%)$ \\
\hline Fusiform & $152(35.10 \%)$ \\
\hline Conical & $26(6.00 \%)$ \\
\hline Marginal roll & $18(4.16 \%)$ \\
\hline Discoid & $17(3.94 \%)$ \\
\hline Ovoid & $10(2.31 \%)$ \\
\hline Cylindrical & $08(1.85 \%)$ \\
\hline Rosette & $08(1.85 \%)$ \\
\hline Fold & $06(1.38 \%)$ \\
\hline Coalescent & $1(0.23 \%)$ \\
\hline Pineapple-like & $1(0.23 \%)$ \\
\hline Lineal & $1(0.23 \%)$ \\
\hline Hemispherical & $1(0.23 \%)$ \\
\hline Claviform & $1(0.23 \%)$ \\
\hline Amorphous & $1(0.23 \%)$ \\
\hline
\end{tabular}

TABLE VIII

\begin{tabular}{cc}
\hline Gall color & Nr. of gall morphotypes \\
\hline Green or greenish & $300(58.14 \%)$ \\
Brown or brownish & $136(26.35 \%) 26.36$ \\
Red or reddish & $40(7.75 \%)$ \\
Yellow or yellowish & $33(6.39 \%)$ \\
Whittish & $05(0.97)$ \\
Orange & $01(0.2 \%)$ \\
Purple & $01(0.2 \%)$ \\
\hline
\end{tabular}

TABLE IX

Galling insect

Nr. of gall morphotypes $(n=234)$

$\begin{array}{cc}\text { Diptera - Cecidomyiidae } & 152(64.96 \%) \\ \text { Diptera - Tephritidae } & 02(0.85 \%) \\ \text { Diptera - Muscomorpha } & 04(1.71 \%) \\ \text { Lepidoptera } & 52(22.22 \%)\end{array}$


TABLE IX (continuation)

\begin{tabular}{cc}
\hline Galling insect & Nr. of gall morphotypes $(\mathbf{n}=\mathbf{2 3 4})$ \\
\hline Hemiptera & $16(6.84 \%)$ \\
Coleoptera & $03(1.28 \%)$ \\
Hymenoptera & $03(1.28 \%)$ \\
Thysanoptera & $02(0.85 \%)$ \\
\hline
\end{tabular}

TABLE X

\begin{tabular}{ccc}
\hline Guild & Insect taxon & $\begin{array}{c}\text { Nr. of gall morphotypes } \\
(\mathbf{n}=\mathbf{1 2 2})\end{array}$ \\
\hline Parasitoids & Hymenoptera & $99(81.1 \%)$ \\
Successors & Thysanoptera & $02(1.6 \%)$ \\
& Formicidae (Hymenoptera) & $02(1.6 \%)$ \\
& Psocoptera & $01(<1.0 \%)$ \\
Inquilines & Diptera & $09(7.3 \%)$ \\
& Lepidoptera & $07(5.7 \%)$ \\
& Coleoptera & $06(4.9 \%)$ \\
& Thysanoptera & $05(4.1 \%)$ \\
\end{tabular}

TABLE XI

\begin{tabular}{|c|c|c|}
\hline \multirow{2}{*}{ Cecidomyiidae genera } & \multicolumn{2}{|c|}{ Number of species } \\
\hline & determined & undetermined \\
\hline Clinodiplosis Kieffer, 1894 & 0 & 8 \\
\hline Alycaulus Rübsaamen 1916 & 1 & 0 \\
\hline Asphondylia Loew, 1850 & 2 & 1 \\
\hline Brugmmania Tavares, 1906 & 0 & 2 \\
\hline Contarinia Rondani, 1860 & 0 & 5 \\
\hline Dasineura Rondani, 1840 & 0 & 2 \\
\hline Liodiplosis Gagné, 2001 & 3 & 0 \\
\hline Lopesia Rübsaamen, 1908 & 0 & 2 \\
\hline Neolasioptera Felt, 1908 & 1 & 2 \\
\hline Perasphondylia Möhn, 1960 & 1 & 0 \\
\hline Schismatodiplosis Rübsaamen, 1916 & 1 & 0 \\
\hline Zalepidota Rübsaamen, 1908 & 0 & 1 \\
\hline Total & 9 & 23 \\
\hline
\end{tabular}


TABLE XII

\begin{tabular}{|c|c|c|}
\hline \multicolumn{2}{|r|}{ Host plant } & \multirow{2}{*}{ Galling family } \\
\hline Family & Genus or species & \\
\hline Apocynaceae & Fischeria DC.sp. & Cecidomyiidae \\
\hline Apocynaceae & Prestonia bahiensis Müll.Arg. & Cecidomyiidae \\
\hline Asteraceae & Baccharis grandimucronata Malag. & Cecidomyiidae \\
\hline Asteraceae & Chromolaena laevigata (Lam.) R.M.King and H.Rob. & Cecidomyiidae \\
\hline Asteraceae & Critonia cf. morifolia (Mill.) R.M.King and H.Rob. & Cecidomyiidae \\
\hline Asteraceae & Mikania buddleiaefolia DC. & Cecidomyiidae \\
\hline Asteraceae & M. hirsutissima DC. & Cecidomyiidae \\
\hline Asteraceae & M. lasiandra DC. & Cecidomyiidae \\
\hline Asteraceae & M. pseudohoffmanniana G. M. Barroso & Cecidomyiidae \\
\hline Asteraceae & Piptocarpha leprosa (Less.) Baker & Cecidomyiidae \\
\hline Asteraceae & Verbesina glabrata Hook. and Arn. & Cecidomyiidae \\
\hline Asteraceae & Vernonanthura H. Rob. & Tephritidae \\
\hline \multirow[t]{2}{*}{ Bignoniaceae } & Dolichandra unguis-cati (L.) L.G. & Muscomorpha \\
\hline & Mansoa difficilis (Cham.) Bureau and K.Schum. & Cecidomyiidae \\
\hline Convolvulaceae & Dicranostyles Benth. sp. & Cecidomyiidae \\
\hline Convolvulaceae & Ipomoea $\mathrm{L}$ & Cecidomyiidae \\
\hline Fabaceae & Inga grandiflora Ducke. & Cecidomyiidae \\
\hline Fabaceae & Inga cf. sessilis (Vell) Mart. & Cecidomyiidae \\
\hline Fabaceae & Mimosa melanocarpa Benth. & Cecidomyiidae \\
\hline Lamiaceae & Aegiphila Jacq. & Cecidomyiidae \\
\hline Loranthaceae & Struthanthus pentamerus Rizzini & Cecidomyiidae \\
\hline Loranthaceae & S. concinnus (Mart.) Mart. & Cecidomyiidae \\
\hline Melastomataceae & Leandra hirta Raddi & Cecidomyiidae \\
\hline Melastomataceae & Miconia ceramicarpa (DC.) Cogn. & Cecidomyiidae \\
\hline Melastomataceae & M. chrysophylla (Rich.) Urb & Cecidomyiidae \\
\hline Melastomataceae & M. cuspidata Naudin & Cecidomyiidae \\
\hline Melastomataceae & T. semidecandra (Schrank and Mart. ex DC.) Cogn. & Cecidomyiidae \\
\hline Menispermaceae & Disciphania Eichler & Cecidomyiidae \\
\hline Myrtaceae & Eugenia schottiana O.Berg. & Cecidomyiidae \\
\hline Nyctaginaceae & Neea oppositifolia Ruiz and Pav. & Cecidomyiidae \\
\hline Piperaceae & Piper marginatum Jacq. & Cecidomyiidae \\
\hline Piperaceae & P. richardiifolium Kunth. & Cecidomyiidae \\
\hline Primulaceae & Myrsine lineata (Mez) Imkhan. & Cecidomyiidae \\
\hline Rosaceae & Prunus myrtifolia (L.) Urb. & Cecidomyiidae \\
\hline Rubiaceae & Borreria tenera DC. & Cecidomyiidae \\
\hline Rubiaceae & Ixora L. & Cecidomyiidae \\
\hline Rubiaceae & Cupania cinerea Poepp. and Endl. & Cecidomyiidae \\
\hline Sapindaceae & Serjania deflexa Gardner & Cecidomyiidae \\
\hline Sapindaceae & S. paucidentata DC. & Cecidomyiidae \\
\hline Solanaceae & Acnistus arborescens (L.) Schltd. & Cecidomyiidae \\
\hline Solanaceae & Solanum megalochiton Mart. & Cecidomyiidae \\
\hline Solanaceae & S. scuticum M.Ne or piluliferum Dunal & Cecidomyiidae \\
\hline Solanaceae & Solanum L. & Muscomorpha \\
\hline
\end{tabular}


TABLE XIII

\begin{tabular}{|c|c|c|c|}
\hline Cecidomyiidae & Life habit & & ost plant \\
\hline Genera & Galler & Family & Genera or species \\
\hline Asphondylia Loew, & Galler & Apocynaceae & Fischeria DC. \\
\hline Asphondylia & Galler & Apocynaceae & Schubertia Mart. \\
\hline Asphondylia & Galler & Nyctaginaceae & Neea oppositifolia Ruiz and Pav. \\
\hline Asphondylia & Galler & Salicaceae & Casearia aculeata Jacq. \\
\hline $\begin{array}{l}\text { Clinodiplosis } \\
\text { Kieffer, } 1894\end{array}$ & Galler & Asteraceae & Mikania Willd. \\
\hline Clinodiplosis & Galler & Primulaceae & Myrsine L. \\
\hline Clinodiplosis & Galler & Solanaceae & Solanum L. \\
\hline Clinodiplosis & Inquiline & Apocynaceae & Fischeria DC. \\
\hline Clinodiplosis & Inquiline & Asteraceae & Mikania glomerata Spreng. \\
\hline $\begin{array}{c}\text { Contarinia } \\
\text { Rondani, } 1860\end{array}$ & Galler & Melastomataceae & Miconia Ruiz and Pav. \\
\hline Contarinia & Galler & Piperaceae & Piper L. \\
\hline Contarinia & Inquiline & Asteraceae & Mikania glomerata Spreng. \\
\hline $\begin{array}{l}\text { Dasineura Rondani, } \\
1840\end{array}$ & Galler & Myrtaceae & Myrcia sylvatica (G.Mey.) DC. \\
\hline $\begin{array}{c}\text { Lopesia } \\
\text { Rübsaamen, } 1908\end{array}$ & Galler & Melastomataceae & Bellucia Raf. \\
\hline Lopesia & Galler & Melastomataceae & Clidemia D. Don \\
\hline $\begin{array}{c}\text { Zalepidota } \\
\text { Rübsaamen, } 1908\end{array}$ & Galler & Piperaceae & Piper tuberculatum Jacq. \\
\hline
\end{tabular}

Solanum L. (Solanaceae) for the first time (Table XII).

New interactions among six genera of Cecidomyiidae (gallers and inquilines) and host plants were also reported and they include determined and undetermined species. The later probably are undescribed gall midges (Table XIII).

The gall morphological characterization (host plant organ, gall shape, color and number of internal chambers), as well as the inducers, associated fauna, paths and dates of collection are presented below. The data was organized by family plant, genus and species in alphabetical order. The number of gall morphotypes for each botanical taxon is given in brackets, as in the following example: Acanthaceae $(n=5)$. New records of the geographical distribution of plant genera and species as well as those of the host plants are indicated. The previous gall records in each plant species or genus were added, following this sequence: biome, host plant (when applicable), reference, number of recorded gall morphotypes, locality and Brazilian state), as in this example: in Pampa: on Justicia sp. - Tavares 1909 (n=1/São Leopoldo/RS).

\section{ACANTHACEAE $(\mathrm{N}=5)$}

Justicia L. sp. (native genus) $(\mathrm{n}=2)$

Gall(Fig. 2): on stem, fusiform, green, glabrous, and one-chambered. Galler: not determined. Path: Travessia Ruy Braga, 08/VII/2014, 16/III/2015.

Gall (Fig. 3): on leaf, conical, green, with trichomes, apically opened, and one-chambered. Galler: Hemiptera. Path: BR-485, 26/01/2015.

Previous gall records on this genus: 1) in Pampa: on Justicia sp. - Tavares 1909 (n=1/ São Leopoldo/RS), 2) no biome data: on Justicia brasiliana Roth - Mendonça et al. 2014 (n=2/RS)

Mendoncia hoffmannseggiana Nees (native species, NE) ( $\mathrm{n}=2)$

(first plant record in Southeast Region and in Atlantic forest) 
Gall (Fig. 4): on leaf and stem, globoid, green, with trichomes, and one-chambered. Galler: not determined. Parasitoids: Hymenoptera (02 adults). Path: Lago Azul, 18/III/ 2014.

Gall(Fig. 5): on stem, globoid, brown, glabrous, and one-chambered. Galler: not determined. Path: Donati-Simon 1, 08/IX/2014.

No previous gall records on this plant species.

Ruellia L.sp. (native genus) ( $\mathrm{n}=1$ )

Gall (Fig. 6): on leaf vein, fusiform, green, glabrous, and one-chambered. Galler: Cecidomyiidae (01 larva). Path: Travessia Ruy Braga, 04/VIII/2014.

Previous gall records on this plant genus: no bioma data, on Ruellia sp. - Mendonça et al. 2014 $(n=1 / R S)$.

\section{ANACARDIACEAE $(\mathrm{N}=3)$}

Spondias L. sp. (native genus) $(\mathrm{n}=1)$

Gall (Fig. 7): on leaf, marginal roll, green, glabrous, and one-chambered. Galler: Hemiptera. Path: Travessia Ruy Braga, 16/III/2015.

No previous gall records on this plant genus.

Tapirira guianensis Aubl. (native species, NE) $(\mathrm{n}=2)$

Gall(Fig. 8): on stem, globoid, brown, glabrous, and one-chambered. Galler: not determined. Path: Viúva Hansen, 10/IX/2014.

Gall (Fig. 9): on leaf, marginal roll, green, glabrous, and one-chambered. Galler: not determined. Inquilines: Hemiptera (03 specimens). Paths: Ecoarte-Lago Azul, 08/IV/2014; DonatiSimon 2, 08/IX/2014; Viúva Hansen, 10/IX/2014.

Previous gall records on this plant species: 1) in Atlantic forest: Maia et al. 2008 ( $n=1 /$ Bertioga/ SP), Santos et al. 2011b ( $\mathrm{n}=1 /$ six municipalities/ PE) in Cerrado: Saito and Urso-Guimarães 2012 ( $n=1 /$ Jataí/SP), Urso-Guimarães et al. $2003(n=1 /$ Delfinópolis/MG), Urso-Guimarães and ScareliSantos 2006 (n=1/Santa Rita do Passa Quatro/MG),
3) in Amazonian forest: Almada and Fernandes 2011 (n=1/Oriximiná/PA).

\section{ANNONACEAE $(\mathrm{N}=3)$}

Duguetia A. St.-Hill. sp. (native genus) $(\mathrm{n}=2)$

Gall (Fig. 10): on leaf midvein, fusiform, green, glabrous, and one-chambered. Galler: not determined (empty galls). Path: Cachoeira Poranga, 07/IV/2014.

Gall (Fig. 11): on leaf, globoid gall, onechambered, yellow, reddish or green. Galler: not determined (empty galls). Paths: Cachoeira Poranga, 07/IV/2014; Travessia Ruy Braga, 10-12/ XI/2014; Casa 33, 16/VI/2015.

Previous gall records on this plant genus: 1) in Atlantic forest: on Duguetia furfuracea (A.St.-Hil.) Saff. - Maia 2013b (n=1/São Tomé das Letras/MG), Fernandes et al. 1997 (n=2/Vale do Jequitinhonha/ MG), 2) in Cerrado: on D. furfuracea - UrsoGuimarães et al. 2003 (n=1/Delfinópolis/MG), Saito and Urso-Guimarães 2012 ( $\mathrm{n}=5 /$ Jataí/SP), Malves and Frieiro-Costa 2012 (n=1/Luiz Antonio/MG), Coelho et al. 2013b ( $\mathrm{n}=1 /$ Serra do Cabral/MG), Urso-Guimarães and Scareli-Santos 2006 (n=3/ Santa Rita do Passa Quatro/MG), Araújo et al. 2015 ( $\mathrm{n}=1 / \mathrm{GO}), 3$ ) in Caatinga-Cerrado transition: on $D$. furfuracea - Costa et al. 2015 ( $\mathrm{n}=1 /$ Caetité/BA), 4) in Amazonian forest: on Duguetia stelechantha (Diels) R.E.Fr. - Almada and Fernandes 2011 (n=1/ Oriximiná/PA), on Duguetia sp. - Araújo et al. 2012 (n=1/ Oriximiná/PA).

Guatteria latifolia (Mart.) R. E. Fr (endemic in Brazil, only in Atlantic forest, NE) $(n=1)$

Gall (Fig. 12): on leaf, globoid, green or reddish, glabrous, and one-chambered. Galler: not determined. Parasitoids: Hymenoptera (1 adult). Path: Casa 33, 16/VI/2015.

No previous gall records on this plant species. 
APOCYNACEAE $(\mathrm{N}=10)$

Ditassa crassifolia Decne. (endemic in Brazil, Cerrado and Atlantic forest, NE) $(n=1)$

(first record in RJ and ombrophylous forest)

Gall (Fig. 13): on stem, fusiform, brown, glabrous, and one-chambered. Galler: not determined. Path: Hotel Donati, 04/VIII/2014.

No previous gall records on this plant species. Fischeria DC. sp.1 (native genus) $(\mathrm{n}=2)$

Gall (Fig. 14): on leaf petiole and vein, fusiform, green, hairy, and one-chambered or multichambered. Galler: Cecidomyiidae (03 young larvae). Parasitoids: Hymenoptera (01 larva, 02 pupas). Inquilines: Coleoptera (01 larva). Successors: Psocoptera (01 adult). Paths: Três Picos, 13/V/2014, 08/IX/2014; Água BrancaRuy Braga, 18/VI/2014, 14/IV/2015, 17/III/2015; Travessia Ruy Braga, 08/VII/2014; Casa 33, 16/ $\mathrm{VI} / 2015$.

Gall (Fig. 15): on leaf, globoid, green, micropubescent, and one or two-chambered. Galler: Asphondylia sp. (Cecidomyiidae) (01 larva). Parasitoids: Hymenoptera (01 adult, 01 larva, 02 pupae). Inquilines: Clinodiplosis sp. (Cecidomyiidae) (03 larvae). Paths: Três Picos, 13/V/2014, 08/IX/2014; Água Branca-Ruy Braga, 18/VI/2014; Travessia Ruy Braga, 08/VII/2014, 04/VIII/2014, 14/IV/2015; Donati-Simon 2, 08/ IX/2014; Viúva Hansen, 10/IX/2014; Casa 33, 16/ $\mathrm{VI} / 2015$.

Fischeria DC. sp.2 ( $\mathrm{n}=1)$

Gall (Fig. 16): on stem, globoid, green, glabrous, and with longitudinal grooves. Galler: Muscomorpha (02 puparia) Path: BR-485, 12/ $\mathrm{XI} / 2014$.

No previous gall records on this plant genus.

Prestonia bahiensis Müll.Arg. (endemic in Brazil, Caatinga, Cerrado and Atlantic forest, LC) (first record in RJ and ombrophilous forest) $(n=1)$

Gall (Fig. 17): on stem and leaf petiole, fusiform, brown, and with brown pubescence.
Galler: Cecidomyiidae (05 adults). Path: Cachoeira Véu da Noiva, 17/III/2014.

No previous gall records on this plant species. Schubertia Mart. sp. (native genus) (first occurrence in RJ and in ombrophilous forest) $(n=4)$

Gall (Fig. 18): on bud, globoid, unilateral, brown, and one-chambered. Galler: Asphondylia sp. (Cecidomyiidae) (03 pupae, 02 females). Paths: Travessia Serra Negra, 14-15/X/2014; BR-485, 23 / II/2015; Cachoeira do Pitu, 25/II/2015.

Gall (Fig. 19): on stem, fusiform, brown, with trichomes, and multichambered. Galler: not determined. Parasitoids: Hymenoptera (01 adult). Path: Três Picos, 08/IX/2014.

Gall (Fig. 20): on leaf vein, globoid, green, glabrous, and one-chambered. Galler: not determined. Parasitoids: Hymenoptera (01 larva, 01 pupa). Paths: Cachoeira Itaporani, 12/V/2014; Travessia Ruy Braga, 24/II/2015.

Gall (Fig. 21): on leaf petiole and vein, fusiform, greenish, and glabrous. Galler: not determined. Parasitoids: Hymenoptera (01 larva). Paths: Travessia Serra Negra, 14-15/X/2014; Travessia Ruy Braga, 24/II/2015.

Previous gall records on this plant genus: 1) in Atlantic forest: on Schubertia grandiflora Mart. Santos et al. 2012 ( $\mathrm{n}=1 / \mathrm{PE})$.

Tabernaemontana L. sp. (native genus) $(\mathrm{n}=1)$

Gall (no fig.): on leaf, globoid, yellow, with basally white and apically red trichomes, and onechambered. Galler: not determined (empty galls). Path: Três Picos, 11/XI/2014.

Previous gall records: 1) in Atlantic forest: on Tabernaemontana sp. - Maia et al. $2014(\mathrm{n}=1 /$ Santa Teresa/ES), Fernandes et al. 2001 ( $n=1 /$ Vale do Rio Doce/MG), 2) in Amazonian forest: on Tabernaemontana catharinensis A. DC. - Araújo et al. 2012 ( $\mathrm{n}=1 /$ Oriximiná/PA).

\section{AQUIFOLIACEAE $(\mathrm{N}=2)$}

Ilex taubertiana Loes. (endemic in Brazil, only in Atlantic forest, NE) $(n=1)$ 

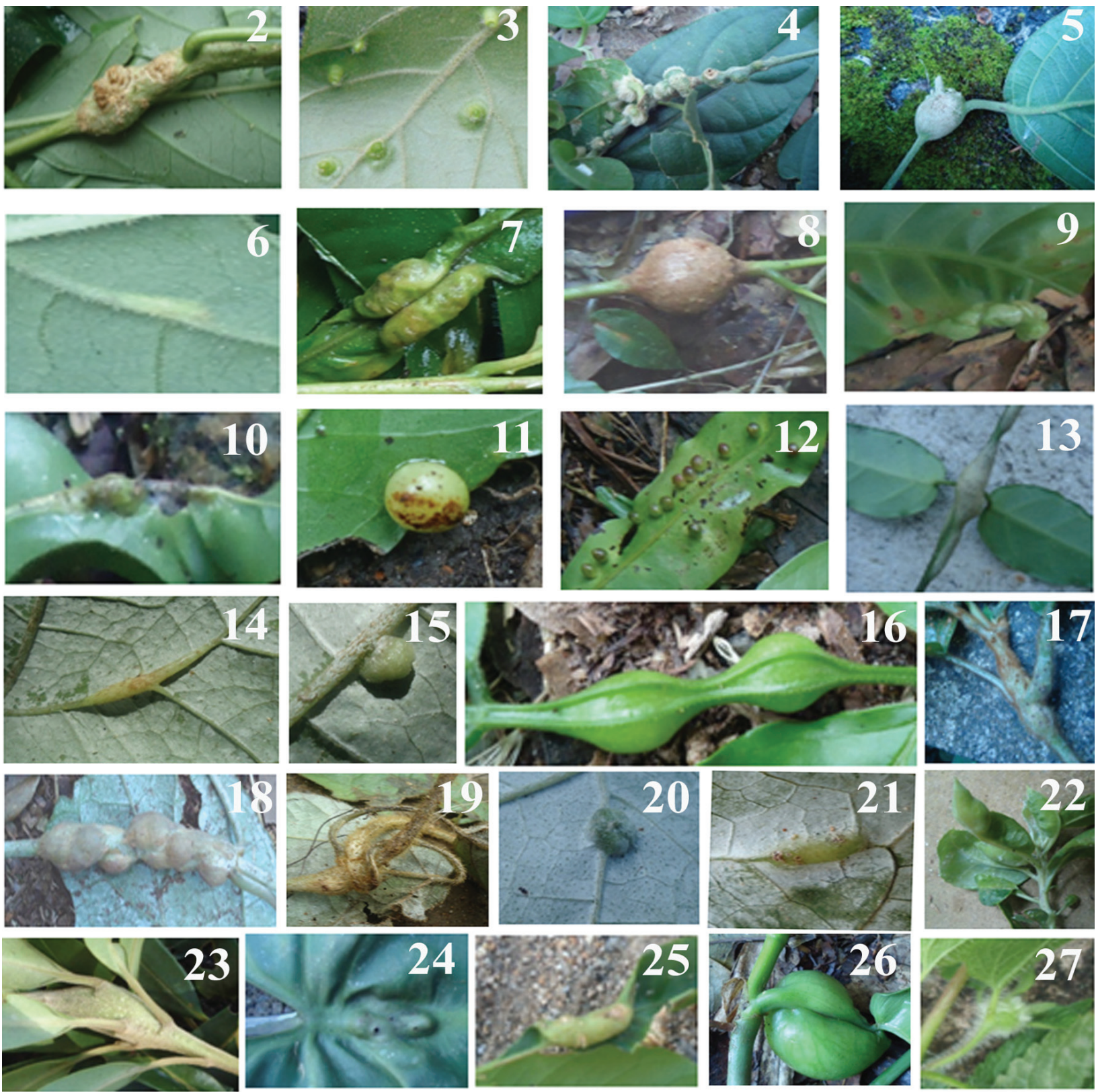

Figure 2-27 - Insect galls of the Parque Nacional do Itatiaia (Southeast Region, Brazil), 2-6, on Acanthaceae, 2-3, Justicia sp., 2, fusiform stem gall, 3, conical leaf gall, 4-5, Mendoncia hoffmannseggiana, 4, globoid leaf and stem gall, 5, globoid stem gall, 6, Ruellia sp., globoid stem gall, 7-9, on Anacardiaceae, 7, Spondias sp., marginal leaf roll, 8-9, Tapirira guianensis, 8, stem globoid gall, 9, marginal leaf roll, 10-12, on Annonaceae, 10-11, Duguetia sp., 10, fusiform leaf gall, 11, globoid leaf gall, 12, Guatteria latifolia, globoid leaf gall, 13-21, on Apocynaceae, 13, Ditassa crassifolia, fusiform stem gall, 14-15, Fischeria sp.1, 14, fusiform leaf petiole and vein gall, 15, globoid leaf gall, 16, Fischeria sp.2, globoid stem gall, 17, Prestonia bahiensis, fusiform stem and leaf petiole gall, 18-21, Schubertia sp., 18, globoid bud gall, 19, fusiform stem gall, 20, globoid leaf vein gall, 21, fusiform leaf petiole and vein gall, 22-23, on Aquifoliaceae, 22, Ilex taubertiana, marginal leaf roll, 23, Ilex sp., fusiform bud gall, 24-26, on Araceae, 24, Philodendron cordatum, fusiform leaf vein gall, 25, P. rudgeanum, marginal leaf roll, 26, Philodendron sp., fusiform bud gall, 27, on Asteraceae, Ageratum conyzoides, globoid bud gall. 
Gall (Fig. 22): on leaf, marginal roll, green, glabrous, and one-chambered. Galler: Hemiptera. Path: Três Picos, 17/III/2015.

No previous gall records on this plant species. Ilex L. sp. (native genus) $(\mathrm{n}=1)$

Gall (Fig. 23): on bud, fusiform, brown, with brown trichomes, and one-chambered. Galler: Lepidoptera (01 catterpilar). Paths: Casa de Pedra, 25/II/2014; Pedra do Camelo 14/IX/2015.

Previous gall records on this genus plant: 1) in Atlantic forest: on I. ceracifolia Reiss. Fernandes et al. 2001 ( $n=1 /$ Vale do Rio Doce/MG), on Ilex microdonta Reiss. - Toma and Mendonça 2013 (n=3/São Francisco de Paula/RS), on Ilex pseudobuxus Reissek - Maia et al. 2008 (n=2/ Bertioga/SP), on Ilex theezans Mart. - Maia et al. 2008 ( $\mathrm{n}=3 /$ Bertioga/SP), on Ilex sp. - Bregonci et al. 2010 ( $n=1 /$ Guarapari/ES), Maia and Oliveira 2010 ( $n=1 /$ Angra dos Reis/RJ), Maia et al. 2014 ( $n=1 /$ Santa Teresa/ES), 2) in Amazonian forest: on Ilex inundata Poepp. ex Reissek - Almada and Fernandes 2011 (n=1/Oriximiná/PA), 3) in Cerrado: on Ilex amara (Vell.) Loes. - Carneiro et al. 2009b ( $\mathrm{n}=1 /$ Cadeia do Espinhaço/MG), on Ilex brasiliensis Loes. - Carneiro et al. 2009b ( $\mathrm{n}=1$ / Cadeia do Espinhaço/MG), 4) no biome data: on Ilex brevicuspis Reissek ( $\mathrm{n}=2 / \mathrm{RS})$, I. microdonta Reissek, I. theezans Mart. ex Reissek ( $\mathrm{n}=2 / \mathrm{RS}$ ) (Mendonça et al. 2014).

\section{$\operatorname{ARACEAE}(\mathrm{N}=3)$}

Philodendron cordatum Kunth ex Schott (endemic in Brazil, only in Atlantic forest, NE) $(n=1)$

Gall (Fig.24): on leaf vein, fusiform, green, glabrous, and multichambered. Galler: Hymenoptera. Paths: Lago Azul, 18/III/2014; BR485, 28/I/2015.

No previous gall records on this plant species. Philodendron rudgeanum Schott (native species, $\mathrm{NE})$ (first record in RJ) $(\mathrm{n}=1)$
Gall (Fig. 25): on leaf, marginal roll, green, glabrous, and one-chambered. Galler: Hemiptera. Parasitoids: Hymenoptera. Paths: Cachoeira Itaporani, 12/V/2014; Travessia Ruy Braga, 14/ IV/2015, 15/IX/2015.

No previous gall records on this plant species. Philodendron Schott. sp. $(\mathrm{n}=1)$

Gall(Fig. 26): on bud, fusiform, green, glabrous, and multichambered. Galler: Muscomorpha (01 adult). Parasitoids: Hymenoptera (01 pupa, 02 adults). Path: Três Picos, 11/XI/2014.

No previous gall records on this plant genus.

\section{ASTERACEAE $(\mathrm{N}=93)$}

Ageratum conyzoides L. (native species, NE) (confirmed occurrence in RJ) $(\mathrm{n}=1)$

Gall (Fig. 27): on bud, globoid, green, with trichomes, and three-chambered. Galler: Cecidomyiidae (03 larvae, 01 pupa, 01 adult). Inquilines: Aphidae (20 specimens), Muscomorpha (01 larva). Path: Ecoarte-Lago Azul, 08/IV/2014.

Previous gall records on this plant species: in Cerrado - Maia and Araújo 2016 (n=1/Dores do Indaiá/MG).

Baccharis altimontana $\mathrm{G}$. Heiden et al. (endemic in Brazil, only in Atlantic forest, exclusively in altitude fields, NE) $(\mathrm{n}=1)$

Gall (Fig. 28): on vein, fusiform, green, glabrous, and one-chambered. Galler: Lepidoptera (01 catterpilar). Path: Travessia Ruy Braga, 13/V/2015.

No previous gall records on this plant species. Baccharis grandimucronata Malag. (endemic in Brazil, only in Atlantic forest, exclusively in altitude fields, LC) $(\mathrm{n}=1)$

Gall (Fig. 29): on leaf, globoid, green, glabrous, and one-chambered. Galler: Cecidomyiidae (01 pupa). Path: Morro do Couto, 05/X/2015.

No previous gall records on this plant species. Baccharis reticularia DC. (endemic in Brazil, Caatinga, Cerrado and Atlantic Forest, NE) $(n=1)$ 
Gall (Fig. 30): on bud and stem, ovoid, brown, glabrous, and one-chambered. Galler: Lepidoptera (02 catterpilars). Inquilines: Sciaridae (01 pupal exuvia, 01 adult). Successors: Formicidae (Hymenoptera) (01 adult). Paths: Agulhas Negras, 18/III/2014; Cachoeira do Aiuruoca, 19/III/2014; Pedra do Altar, 08/IV/2014; Prateleiras, 09/ IV/2014; Morro do Couto, 13/V/2014; Travessia Serra Negra, 14-15/X/2014; Pedra da Maçã, Tartaruga and Assentada, 27/I/2015; Cinco Lagos, 11-12/V/2015; Travessia Ruy Braga, 13/V/2015; Ovos da Galinha, 13/V/2015; Pedra do Camelo, 14/ IX/2015; Asa de Hermes, 16/IX/2015.

Previous gall records on this plant species: in Cerrado - Maia and Fernandes 2004 (n=3/Serra de São José/MG), Carneiro et al. 2009b (n=7/Cadeia do Espinhaço/MG).

Baccharis L. sp. $(\mathrm{n}=5)$

Gall (Fig. 31): on leaf, globoid, green, with trichomes, and one-chambered. Galler: Cecidomyiidae (03 pupal exuviae, 03 adults). Parasitoids: Hymenoptera (01 larva, 04 adults). Paths: Travessia Serra Negra, 14-15/X/2014; Travessia Ruy Braga, 18/VI/2014, 17/III/2015, 14/ IV/2015, 15/IX/2015.

Gall (Fig. 32): on bud, rosette, green, glabrous, and one-chambered. Galler: Cecidomyiidae (02 larvae). Parasitoids: Hymenoptera (01 larva). Paths: Casa de Pedra, 25/ II/2014; Travessia Serra Negra, 14-15/X/2014; Travessia Ruy Braga, 18/ $\mathrm{VI} / 2014,14 / \mathrm{IV} / 2015$.

Gall (Fig. 33): on leaf, marginal roll, green, glabrous, and one-chambered. Galler: not determined (empty galls) (Fig.). Path: Travessia Ruy Braga, 18/VI/2014, 14/IV/2015.

Gall (Fig. 34): on leaf petiole, midvein, and stem, globoid or fusiform, green or yellowish, glabrous, and multichambered. Galler: not determined (empty galls). Path: Travessia Ruy Braga, 18/VI/2014, 14/IV/2015, 15/IX/2015.

Gall (Fig. 35): on stem and leaf petiole, fusiform, brown, and glabrous. Galler: not determined (eggs) (Fig.). Path: Casa de Pedra, 25/ II/2014.

Previous gall records on not determined species of Baccharis: 1) in Atlantic forest - Maia and Oliveira 2010 (n=1/Angra dos Reis/RJ), Maia 2013 b (n=3/São Tomé das Letras/MG), Toma and Mendonça 2013 (n=1/São Francisco de Paula/RS), Maia 2014 (n=2/Itamonte/MG), 2) in Cerrado Carneiro et al. 2009b ( $\mathrm{n}=1 /$ Cadeia do Espinhaço/ MG), 3) no bioma data - Mendonça et al. 2014 $(\mathrm{n}=6 / \mathrm{RS})$.

Barrosoa organensis (Gardner) R.M.King and H. Rob. (endemic in Brazil, Cerrado and Atlantic forest, NE) $(n=1)$

Gall (Fig. 36): on stem, fusiform, green, glabrous, and one-chambered. Galler: not determined. Path: Casa de Pedra, 25/II/2014.

No previous gall records on this plant species. Bidens segetum Mart. ex Colla (native species, $\mathrm{NE})$ (first record in ombrophilous forest $(\mathrm{n}=1)$

Gall (Fig. 37): on stem, fusiform, green and brown, glabrous, and one-chambered. Galler: not determined (empty galls). Path: Travessia Ruy Braga, 24/II/2015.

No previous gall records on this plant genus.

Bidens L. sp. (native genus) $(\mathrm{n}=1)$

Gall (Fig. 38): on bud, globoid, green, glabrous, and one-chambered. Galler: not determined. Path: Casa 16, 07/VII/2014.

No previous gall records on this plant genus. Calea pinnatifida (R. Br.) Less (native species, $\mathrm{NE})(\mathrm{n}=1)$

Gall (Fig. 39): on bud, globoid, green, glabrous, and one-chambered. Galler: not determined (empty galls). Path: BR-485, 09/XII/204.

Previous gall records on this plant species: no biome data - Mendonça et al. 2014 ( $n=2 / R S)$.

Chionolaena lychnophorioides Sch.Bip. (endemic in Brazil, Cerrado, VU) (first record in RJ and Atlantic forest) $(n=1)$

Gall (Fig. 40): on stem, globoid, brown, glabrous, and one-chambered. Galler: not 
determined (empty galls). Path: Cachoeira do Aiuruoca, 19/III/2014.

No previous gall records on this plant species.

Chromolaena laevigata (Lam.) R.M.King and H.Rob. (native species, NE) $(n=2)$

Gall (Fig. 41): on leaf vein, fusiform, green, glabrous, and one-chambered. Galler: Cecidomyiidae (01 young larva). Inquilines: Lepidoptera (01 catterpilar). Paths: Travessia Ruy Braga, 08/XII/2014, 14/IV/2015; Travessia Serra Negra, 14-15/X/2014; Asa de Hermes, 16/IX/2015.

Gall (Fig. 42): on stem, fusiform, green or brown, pubescent, and one-chambered. Galler: not determined (empty galls). Path: Travessia Ruy Braga, 08/XII/2014, 14/IV/2015.

No previous gall records on this plant species. Chromolaena odorata (L.) R.M.King and H.Rob. (native species, NE) $(\mathrm{n}=3)$

Gall (no fig.): on leaf vein, fusiform, green, micropubescent, and one-chambered. Galler: Cecidomyiidae (01 larva). Parasitoids: Hymenoptera (02 pupae). Path: Travessia Ruy Braga, 07/III/2015.

Gall (Fig. 43): on stem, globoid, brown, micropubescent, and one-chambered. Galler: not determined. Path: Travessia Ruy Braga, 14/ IV/2015.

Gall (Fig. 44): on stem, fusiform, green, with white trichomes, and one-chambered. Galler: not determined. Path: Travessia Ruy Braga, 14/ IV/2015.

Previous gall records on this plant species: in Amazonian forest - Gagné 1977, 1994 (n=1/Pará). Critonia cf. morifolia (Mill.) R.M.King and H.Rob. (native species, NE) (first record in RJ/MG and Atlantic forest $)(n=1)$

Gall (Fig. 45): on leaf petiole, fusiform, green, glabrous, and one-chambered. Galler: Cecidomyiidae (01 larva). Paths: Posto MarcãoAbrigo Rebouças, 24/II/2014.

No previous gall records on this plant species.
Dasyphyllum spinescens (Less.) Cabrera (native species, NE) $(\mathrm{n}=1)$

Gall (Fig. 46): on leaf petiole, fusiform, brown, and micropubescent. Galler: Coleoptera (02 larvae). Path: Casa 33, 16/VI/2015.

Previous gall records on this plant species: no biome data - Mendonça et al. 2014 ( $n=2 / R S)$.

Dendrophorbium fruticosum (Vell.) C.Jeffrey (native species, NE) $(\mathrm{n}=2)$

Gall (Fig. 47): on stem, fusiform, green, and glabrous. Galler: Lepidoptera. Path: Travessia Ruy Braga, 15/IX/2015.

Gall (Fig. 48): on leaf, globoid, green, glabrous, and multichambered. Galler: not determined (empty galls). Path: Travessia Ruy Braga, 15/IX/2015.

No previous gall records on this plant species.

Eupatorium L. sp. 1 (native genus) $(\mathrm{n}=4)$

Gall(Fig.49): on stem, fusiform, green or brown, glabrous, and one-chambered or multichambered. Galler: Neolasioptera sp. (Cecidomyiidae) (01 larva). Parasitoids: Hymenoptera (01 larva, 02 pupae). Paths: Três Picos, 13/V/2014, 11/XI/2014; Hotel Donati, 04/VIII/2014; Donati-Simon 2, 08/ IX/2014; Travessia Ruy Braga, 08/XII/2014, 16/ III/2015, 17/III/2015.

Gall (Fig. 50): on stem, globoid, brown, glabrous, and one-chambered. Galler: Contarinia sp. (Cecidomyiidae) (01 larva). Path: Travessia Ruy Braga, 14/IV/2015.

Gall (Fig. 51.): on midvein, globoid, green, micropubescent, and one-chambered. Galler: Cecidomyiidae (03 larvae). Parasitoids: Hymenoptera (02 pupae). Paths: Casa de Pedra, 25/ II/2014; Cachoeira Itaporani, 17 /III/2014; Água Branca-Ruy Braga, 18/VI/2014; Travessia Ruy Braga, 16/III/2015.

Gall (Fig. 52): on leaf petiole and midvein, fusiform, green, micropubescent, one-chambered. Galler: not determined (empty galls). Path: Hotel Donati, 04/VIII/2014.

Eupatorium L. sp. $2(\mathrm{n}=1)$ 

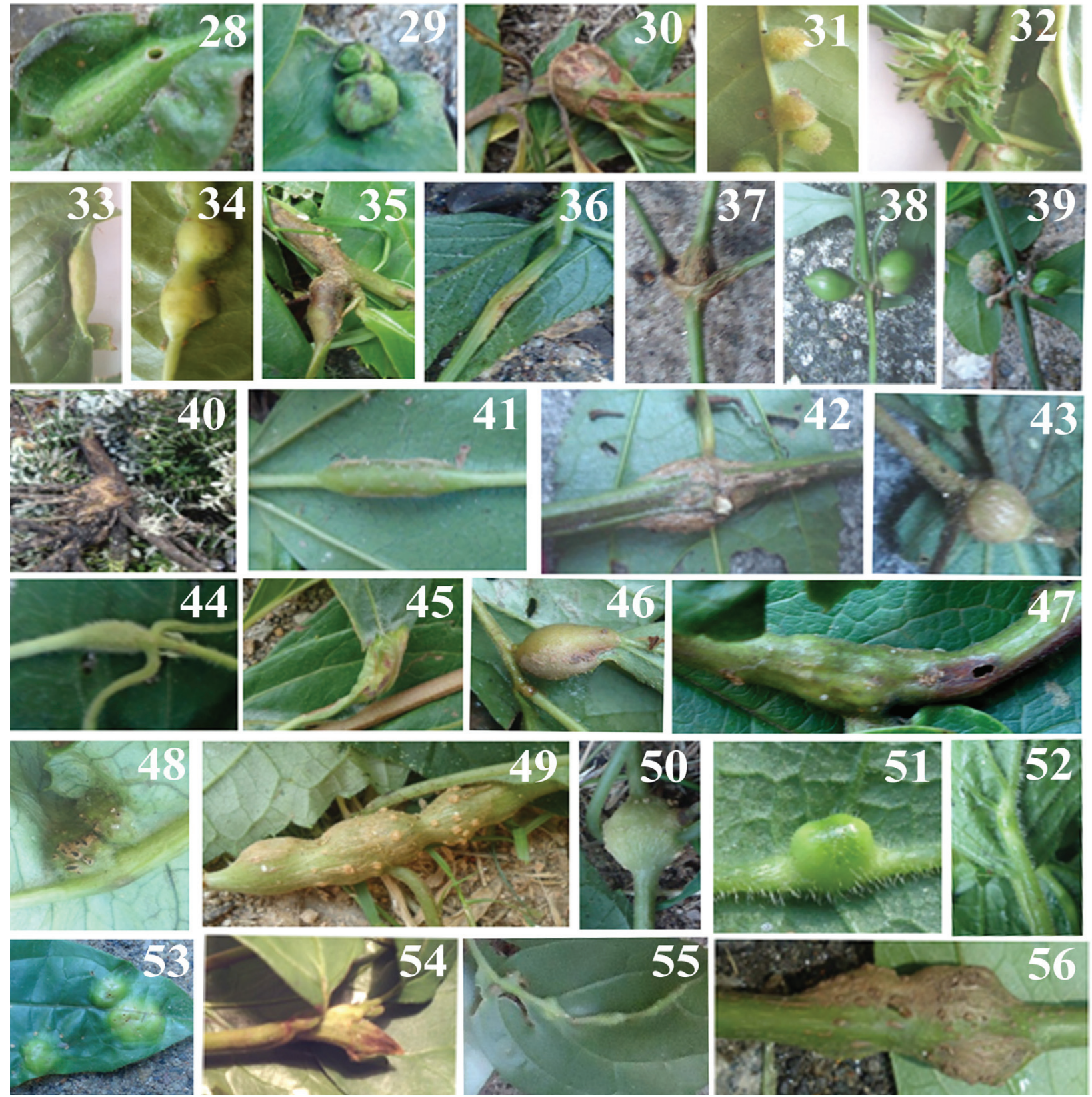

Figure 28-56 - Insect galls of the Parque Nacional do Itatiaia (Southeast Region, Brazil), on Asteraceae, 28, Baccharis altimontana, fusiform leaf vein gall, 29, B. grandimucronata, globoid leaf gall, 30, B. reticularia, ovoid bud and stem gall, 31-35, Baccharis sp., 31, globoid leaf gall, 32, rosette bud gall, 33, marginal leaf gall, 34, globoid midvein gall, 35, fusiform stem and leaf petiole gall, 36, Barrosoa organensis, fusiform stem gall, 37, Bidens segetum, fusiform stem gall, 38, Bidens sp., globoid bud gall, 39, Calea pinnatifolia, globoid bud gall, 40, Chiomolaena lychnophorioides, globoid stem gall, 41-42, Chromoalena laevigata, 41, fusiform leaf vein gall, 42, fusiform stem gall, 43-44, C. odorata, 43, globoid stem gall, 44, fusiform stem gall, 45, Critonia cf. morifolia, fusiform leaf petiole gall, 46, Dasyphyllum spinescens, fusiform leaf petiole gall, 47-48, Dendrophorbium fruticosum, 47, fusiform stem gall, 48, globoid leaf gall, 49-52, Eupatorium sp.1, 49, fusiform stem gall, 50, globoid stem gall, 51, globoid midvein gall, 52, fusiform leaf petiole and midvein gall, 53, Eupatorium sp.2, discoid leaf gall, 54-56, Eupatorium sp.3, 54, cylindrical bud gall, 55, fusiform leaf vein gall, 56, fusiform stem gall. 
Gall (Fig. 53): on leaf, discoid, green or purple, and glabrous. Galler: Cecidomyiidae (01 pupal exuvia, 02 larvae). Paths: Lago Azul, 18/III/ 2014; Cachoeira Itaporani, 12/V/2014; Travessia Ruy Braga, 08/XII/2014; 24/II/2015; BR-485, 09/ XII/2014; Cachoeira do Pitu, 25/II/2015.

Eupatorium L. sp. $3(\mathrm{n}=4)$

Gall (Fig. 54): on bud, cylindrical, green, with brown micropubescence, and one-chambered. Galler: Clinodiplosis sp. (Cecidomyiidae) (02 larvae by gall). Path: Viúva Hansen, 10/IX/2014.

Gall (Fig. 55): on leaf vein, fusiform or globoid, green or reddish, glabrous, and onechambered. Galler: Cecidomyiidae (02 larvae, 01 pupa). Parasitoids: Hymenoptera (03 pupae, 01 adult). Paths: Lago Azul, 18/III/ 2014; Centro de Visitantes, 07-08/VII/2014; Viúva Hansen, 10/IX/2014; Barbosa-Rodrigues (obelisk), 10/ $\mathrm{XII} / 2014$.

Gall (Fig. 56): on stem, fusiform, green or brown, glabrous, and one-chambered. Galler: not determined. Parasitoids: Hymenoptera (fragments). Paths: Barbosa-Rodrigues (obelisk) 10/XII/2014; BR-485, 28/I/2015.

Gall (Fig. 57): on leaf and stem, conical, green, glabrous, and one-chambered. Galler: not determined (empty galls). Paths: Lago Azul, 18/ III/ 2014; BR-485, 04/VIII/2014; Travessia Ruy Braga, 08/XII/2014; Barbosa-Rodrigues (obelisk), 10/XII/2014.

Eupatorium L. sp. $4(\mathrm{n}=1)$

Gall (Fig. 58): on leaf, globoid, yellow, and glabrous. Galler: Cecidomyiidae (01 young larva). Path: Três Picos, 08/IX/2014.

Eupatorium L. sp. $5(\mathrm{n}=1)$

Gall (Fig. 59): on stem, fusiform, brown, glabrous, and one-chambered. Galler:Cecidomiidae (01 larva). Parasitoids: Hymenoptera (02 pupae). Path: Travessia Serra Negra, 14-15/X/2014.

Previous gall records on this plant genus: 1) in Atlantic forest: on Eupatorium squalidum DC. Fernandes et al. 1997 ( $\mathrm{n}=1 /$ Vale do Jequitinhonha/
MG), Maia et al. 2014 ( $\mathrm{n}=1 /$ Santa Teresa/ES), Maia 2014 (n=1/Itamonte/MG), on Eupatorium serratum Spreng. - Toma and Mendonça 2013 (n=1/São Francisco de Paula/RS), 2) no biome data - Mendonça et al. 2014 ( $n=2 / R S)$.

Graphistylis itatiaiae (Dusén) B.Nord. (native species, NE) ( $\mathrm{n}=1)$

Gall (Fig. 60): on apical bud, brown (dried), glabrous, and multichambered. Galler: no determined. Parasitoids: Hymenoptera (fragments). Paths: Travessia Ruy Braga, 13/V/2015, Cinco Lagos, 11-12/V/2015.

No previous gall records on this plant species. Grazielia gaudichaudeana (DC.) R.M.King and H.Rob. (native species, NE) $(n=2)$

Gall (Fig. 61): on leaf, globoid, green, and one-chambered. Galler: not determined. Inquilines: Lepidoptera (01 catterpilar, phytophagous freeliving). Paths: Pedra da Maçã, Tartaruga and Assentada, 27/I/2015; Morro do Couto, 05/X/2015.

Gall (Fig. 62): on bud, rosette, green, glabrous, and one-chambered. Galler: Lepidoptera (03 catterpilars). Paths: Pedra da Maçã, Tartaruga and Assentada, 27/I/2015; Cinco Lagos, 12/05/2015; Travessia Ruy Braga, 13/V/2015, Pedra do Sino, 08/X/2015.

Previous gall records on this plant species: 1) in Atlantic forest (altitude fields) -Coelho et al. 2013a (n=1/Itatiaia).

Mikania buddleiaefolia DC. (endemic in Brazil, Atlantic forest, NE) $(n=2)$

Gall (Fig. 63): on leaf, globoid, adjacent to the midvein, green, glabrous, and one-chambered. Galler: Cecidomyiidae (01 pupa). Path: Travessia Ruy Braga, 08/XII/2014.

Gall (Fig. 64): on leaf petiole, globoid, brown, glabrous, and one-chambered. Galler: not determined. Path: Travessia Ruy Braga, 08/ $\mathrm{XII} / 2014$.

No previous gall records on this plant species. Mikania glomerata Spreng. (native species, LC) $(\mathrm{n}=8)$ 
Gall (Fig. 65): on stem, conical, green, glabrous, and one-chambered. Galler: Asphondylia glomeratae Gagné, 2001 (Cecidomyiidae). Path: Casa 33, 16/VI/2015.

Gall (Fig. 66): on leaf vein, fusiform, green, and one-chambered. Galler: Alycaulus globulus Gagné, 2001 (Cecidomyiidae) (01 pupa). Paths: BR-485, 04/VIII/2014, 12/XI/2014; Viúva Hansen, 10/IX/2014; Casa 25, 08/XII/2015; 23/II/2015. Previous records on the same gall morphotype on this plant species: in Atlantic forest - Gagné et al. 2001 (Silva Jardim, Poço das Antas and PNI/RJ), Oliveira and Maia 2005 (Grumari, Rio de Janeiro, $\mathrm{RJ})$.

Gall (Fig. 67): on stem, fusiform, green, glabrous, and one or multi-chambered. Galler: Asphondylia moehni Skuhravá, 1989 (Cecidomyiidae) (02 larvae). Parasitoids: Hymenoptera (01 pupa, 04 adults). Inquilines: Clinodiplosis sp. (Cecidomyiidae) (01 larva). Paths: Cachoeira Poranga, 07/IV/2014, 24/II/2015; Donati-Simon 2, 08/IX/2014; Travessia Serra Negra, 14-15/X/2014; Centro de Visitantes, 09/ XII/2014; Barbosa Rodrigues-Mirante do Último Adeus, 09/XII/2014; BR-485, 28/I/2015, 23/ II/2015; Casa 33, 16/VI/2015. Previous records on the same gall morphotype on this plant species: in Atlantic forest - Gagné et al. 2001 (Silva Jardim and Poço das Antas/RJ), Oliveira and Maia 2005 (Grumari, Rio de Janeiro, RJ).

Gall (Fig. 68): on leaf blade and petiole, conical, green, glabrous, and one-chambered. Galler: Liodiplosis conica Gagné, 2001 (Cecidomyiidae). Path: Ecoarte-Lago Azul, 08/IV/2014. Previous records on the same gall morphotype on this plant species: in Atlantic forest - Gagné et al. 2001 (Silva Jardim and Poço das Antas/RJ), Oliveira and Maia 2005 (Grumari, Rio de Janeiro, RJ).

Gall (Fig. 69): on leafblade and petiole, globoid, green, glabrous, and one or multichambered. Galler: Liodiplosis spherica Gagné, 2001 (Cecidomyiidae) (07 larvae). Parasitoids: Hymenoptera (02 larvae, 01 adult). Inquilines: Contarinia sp. (Cecidomyiidae) (01 larva), Clinodiplosis sp. (Cecidomyiidae) (02 larvae), Coleoptera (01 larva). Successors: Thysanoptera (15 specimes). Paths: Ecoarte-Lago Azul, 08/IV/2014; Donati-Simon 2, 08/IX/2014; Barbosa Rodrigues-Mirante do Último Adeus, 09/XII/2014; BR-485, 28/I/2015, 23/II/2015; Cachoeira Poranga, 24/II/2015; Travessia Ruy Braga, 24/II/2015; Casa 33, 16/VI/2015. Previous records on the same gall morphotype on this plant species: in Atlantic forest - Gagné et al. 2001 (Silva Jardim and Poço das Antas/RJ).

Gall (Fig. 70): on leaf, cylindrical, green, glabrous, and one-chambered. Galler: Liodiplosis cylindrica Gagné, 2001 (Cecidomyiidae) (04 larvae). Parasitoids: Hymenoptera (02 pupae, 01 adult). Paths: Ecoarte-Lago Azul, 08/IV/2014; Donati-Simon 2, 08/IX/2014; Viúva Hansen, 10/ IX/2014; Travessia Ruy Braga, 10-12/XI/2014, 24/II/2015; BR-485, 12/XI/2014, 26/I/2015, 28/I/2015, 23/II/2015; Barbosa Rodrigues-Mirante do Último Adeus, 09/XII/2014; Cachoeira Poranga, 24/II/2015; Casa 33, 16/VI/2015. Previous records on the same gall morphotype on this plant species: in Atlantic forest - Gagné et al. 2001 (Silva Jardim and Poço das Antas/RJ).

Gall(Fig. 71): on bud, globoid, brown, glabrous, and multichambered. Galler: Perasphondylia mikaniae Gagné, 2001 (Cecidomyiidae). Parasitoids: Hymenoptera (09 adults). Path: Donati-Simon 1, 10/IX/2014. Previous records on the same gall morphotype on this plant species: in Atlantic forest - Gagné et al. 2001 (Silva Jardim, Poço das Antas and PNI/RJ).

Gall (Fig. 72): on stem, cylindrical, brown, glabrous, and multichambered. Galler: not determined. Path: Casa 33, 16/VI/2015.

Mikania hirsutissima DC. (native species, NE) $(\mathrm{n}=2)$

Gall (Fig. 73): on stem and leaf petiole, fusiform, green, pubescent, and one-chambered. 
Galler: Cecidomyiidae (01 larva). Paths: BR-485, 09/XII/2014, Casa 25 08/XII/2015.

Gall (Fig. 74): on leaf blade, petiole and stem, globoid, green, with trichomes, and onechambered. Galler: not determined. Dweller: Hemiptera (01 cochineal). Paths: Água BrancaRuy Braga, 18/VI/2014; Travessia Ruy Braga, 14/ IV/2015, BR-485, 09/XII/2014.

No previous gall records on this plant species. Mikania lasiandra DC. (endemic in Brazil, Cerrado and Atlantic forest, NE) $(n=1)$

Gall (Fig. 75): on stem and leaf, unilateral, globoid, brown, and glabrous. Galler: Cecidomyiiidae (01 larva). Parasitoids: Hymenoptera (fragments). Path: Travessia Ruy Braga, 14/IV/2015.

No previous gall records on this plant species. Mikania lindbergii Baker (endemic in Brazil, Caatinga, Cerrado and Atlantic forest, LC) $(n=1)$

Gall (Fig. 76): on leaf, conical, green, glabrous, and one-chambered. Galler: Cecidomyiidae (02 young larvae). Paths: Água Branca-Ruy Braga, 18/ VI/2014; Viúva Hansen, 10/IX/2014; Cachoeira do Pitu, 25/II/2015.

Previous gall records on this plant species: in Cerrado - Maia and Fernandes 2004 ( $n=1 /$ Serra de São José/MG).

Mikania micrantha Kunth (native species, NE) $(\mathrm{n}=1)$

Gall (Fig. 77): on leaf, green, globoid, glabrous, and one to three-chambered. Galler: Cecidomyiidae. Path: Travessia Ruy Braga, 04/VIII/2014. Previous record of the same gall morphotype: in Cerrado Maia and Fernandes 2004 (Serra de São José/MG).

Gall (Fig. 78): on leaf petiole, fusiform, green, glabrous, and one-chambered. Galler: Cecidomyiidae. Path: Água Branca-Ruy Braga, 18/VI/2014. Previous record of the same gall morphotype: in Atlantic forest - Maia et al. 2008 (Bertioga/SP).
Mikania periplocifolia Hook. and Arn. (native species, NE) (first record in RJ and Atlantic forest) $(\mathrm{n}=1)$

Gall (Fig. 79): on leaf midvein, fusiform, green, glabrous, and one-chambered. Galler: not determined. Parasitoids: Hymenoptera (01 pupa). Path: Centro de Visitantes, 97/VII/2014.

No previous gall records on this plant species. Mikania pseudohoffmanniana G. M. Barroso (endemic in Brazil, Atlantic forest, NE) (first record in $\mathrm{RJ})(\mathrm{n}=2)$

Gall (Fig. 80): on stem, fusiform, green, with tricomes, and one-chambered. Galler: Cecidomyiidae (01 young larva). Parasitoids: Hymenoptera (01 larva, 01 pupa). Paths: Travessia Ruy Braga, 08/VII/2014, 14/IV/2015, 15/IX/2015; Água Branca-Ruy Braga, 18/VI/2014; Centro de Visitantes, 07-08/VII/2014, 09/XII/2014.

Gall (Fig. 81): on bud, globoid, brown, and glabrous. Galler: not determined. Path: Travessia Ruy Braga, 08/VII/2014.

No previous gall records on this plant species. Mikania setigera Sch.Bip. (endemic in Brazil, Cerrado and Atlantic forest, NE) $(n=1)$

Gall (Fig. 82): on leaf vein, fusiform, yellowish, pubescent. Galler: not determined. Path: Travessia Ruy Braga, 14/IV/2015.

No previous gall records on this plant species. Mikania Willd. sp.1 (native genus) $(\mathrm{n}=2)$

Gall (Fig. 83): on leaf, globoid, green, and glabrous. Galler: Cecidomyiidae (04 larvae) (Fig.). Paths: Cachoeira Véu da Noiva, 17/III/2014; Três Picos, 13/V/2014; BR- 485, 23/II/2015.

Gall (no fig.): on leaf petiole, fusiform, green, glabrous, and one-chambered. Galler: Cecidomyiidae (08 larvae). Paths: BR-485, 23/ II/2015.

Mikania Willd. sp.2 ( $\mathrm{n}=1)$

Gall (Fig. 84): on leaf midvein, fusiform, green, micropubescent, and one-chambered. Galler: Cecidomyiidae (01 larva). Path: Três Picos, 13/V/2014. 

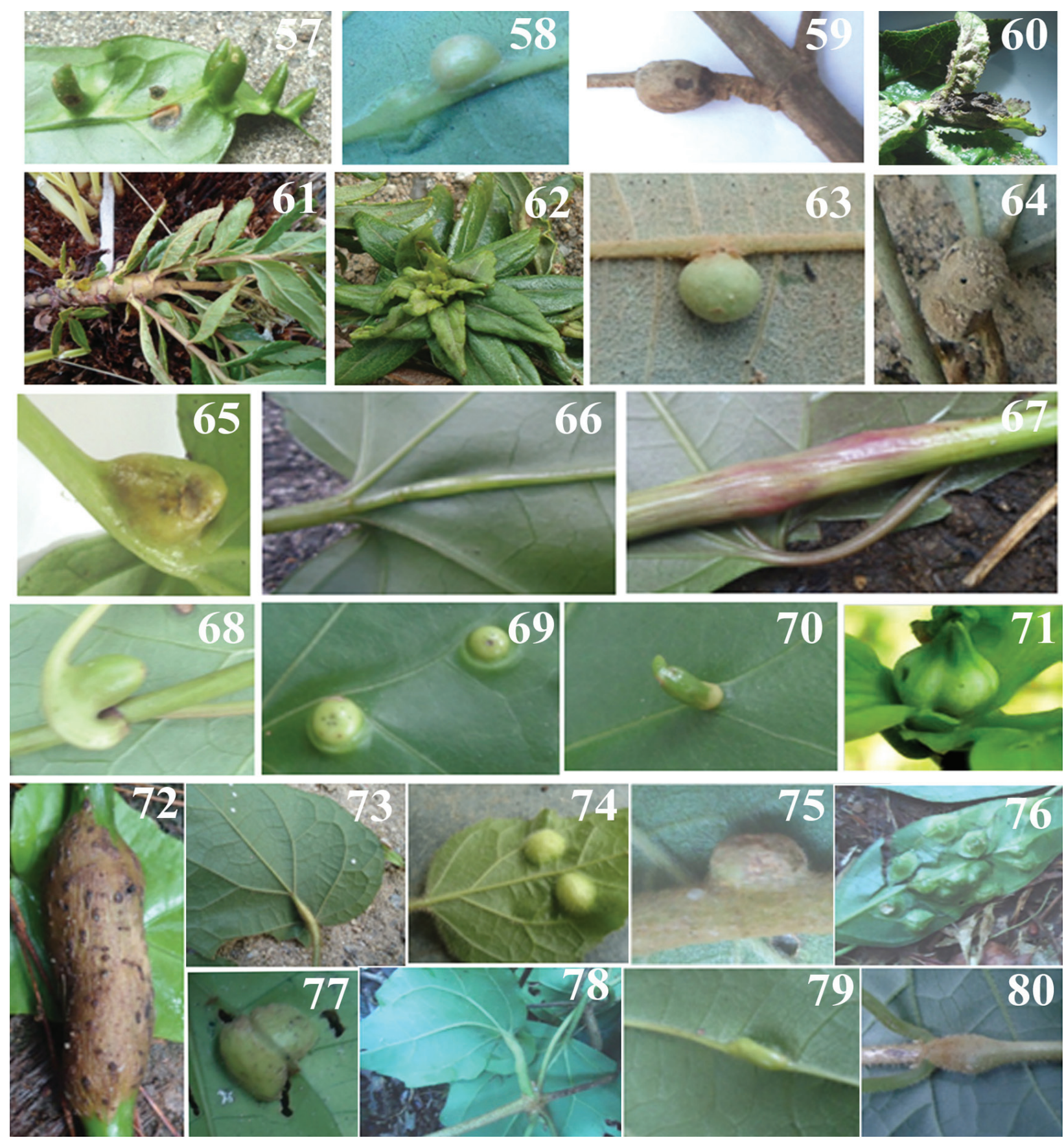

Figure 57-80 - Insect galls of the Parque Nacional do Itatiaia (Southeast Region, Brazil), on Asteraceae, 57, Eupatorium sp.3, fusiform stem gall, 58, Eupatorium sp.4, globoid leaf gall, 59, Eupatorium sp.5, fusiform stem gall, 60, Graphistylis itatiaiae, apical bud gall, 61-62, Grazielia gaudichandeana, 61, globoid leaf gall, 62, rosette bud gall, 63-64, Mikania buddleiaefolia, 63, globoid leaf gall, 64, globoid leaf petiole gall, 65-72, M. glomerata, 65, conical stem gall, 66, fusiform leaf vein gall, 67, fusiform stem gall, 68, conical leaf blade and petiole gall, 69, globoid leaf blade and petiole gall, 70, cylindrical gall, 71, globoid bud gall, 72, cylindrical stem gall, 73-74, M. hirsutissima, 73, fusiform stem and leaf petiole gall, 74, globoid leaf blade, petiole and stem gall, 75, M. lasiandra, globoid leaf and stem gall, 76, M. lindbergii, conical leaf gall, 77-78, M. micrantha, 77, globoid leaf, 78, fusiform leaf petiole, 79, M. periplocifolia, fusiform leaf midvein gall, 80, M. pseudohoffmanniana, fusiform stem gall. 
Mikania Willd. sp. $3(\mathrm{n}=8)$

Gall (Fig. 85): on subterraneous root, fusiform, brown, glabrous, and one-chambered. Galler: Lepidoptera. Path: Água Branca-Ruy Braga, 18/ $\mathrm{VI} / 2014$.

Gall (Fig. 86): on leaf, discoid, green or reddish, glabrous, and one-chambered. Galler: Cecidomyiidae (01 young larva). Paths: Travessia Ruy Braga, 18/VI/2014; Água Branca-Ruy Braga, 18/VI/2014, 14/IV/2015, 15/IX/2015.

Gall (Fig. 87): on leaf, conical, green, glabrous, and one-chambered. Galler: Cecidomyiidae (02 young larvae). Parasitoids: Hymenoptera (05 larvae, 04 pupae). Path: Viúva Hansen, 10/IX/2014.

Gall (Fig. 88): on stem, fusiform or globoid, green or brown, and one-chambered. Galler: Contarinia sp. (Cecidomyiidae) (01 larva). Parasitoids: Hymenoptera (03 larvae, 01 pupa, 04 adults). Paths: Travessia Ruy Braga, 17/III/2015, 14/IV/2015, 18/VI/2014; Travessia Serra Negra, $14-15 / \mathrm{X} / 2014$.

Gall (Fig. 89): on leaf petiole and midvein, fusiform, reddish, pubescent, and one-chambered. Galler: not determined (empty galls). Path: Água Branca-Ruy Braga, 18/VI/2014.

Gall (Fig. 90): on leaf midvein, globoid, green, glabrous, and one-chambered. Galler: Cecidomyiidae (fragments of larva). Paths: Água Branca-Ruy Braga, 18/VI/2014; Travessia Ruy Braga, 08/VII/2014.

Gall (Fig. 91): on bud and stem, globoid, green, glabrous, and one-chambered. Galler: Lepidoptera (01 catterpilar) (Fig.). Path: Água Branca-Ruy Braga, 18/VI/2014, Três Picos, 11/XI/2014.

Gall (Fig. 92): on leaf petiole, fusiform, brown, glabrous, and one-chambered. Galler: not determined. Path: Travessia Ruy Braga, 08/ VII/2014.

Mikania Willd. sp. $4(\mathrm{n}=1)$

Gall (Fig. 93): on stem, leaf petiole and vein, globoid, reddish, glabrous, and one-chambered.
Galler: Clinodiplosis sp. (Cecidomyiidae) (1 larva). Path: Travessia Ruy Braga, 08/VII/2014.

Mikania Willd. sp. $5(\mathrm{n}=2)$

Gall (Fig. 94): on stem, unilateral, green, globoid or fusiform, pubescent, and onechambered. Galler: not determined (empty galls). Path: Hotel Donati, 04/VIII/2014.

Gall (Fig. 95): on leaf vein, globoid, green, pubescent, and one-chambered. Galler: not determined (empty gall). Paths: Hotel Donati, 04/ VIII/2014; Travessia Serra Negra, 14-15/X/2014. Mikania Willd. sp. $6(\mathrm{n}=1)$

Gall (Fig. 96): on bud, rosette of leaves, green, glabrous, and one-chambered. Galler: not determined. Path: BR-485, 04/VIII/2014.

Mikania Willd. sp. $7(\mathrm{n}=1)$

Gall(Fig.97): onstem, fusiform, green, glabrous, and two-chambered. Galler: Cecidomyiidae (02 larvae). Parasitoids: Hymenoptera (02 larvae). Path: BR-485, 04/VIII/2014.

Previous gall records on undetermined species of Mikania: 1) in Atlantic forest - Maia and Oliveira 2010 ( $n=4 /$ Angra dos Reis/RJ), Santos et al. 2011b ( $\mathrm{n}=2 /$ six municipalities/PE) Maia et al. 2014 (n=15/Santa Teresa/ES), Maia 2014 (n=4/ Itamonte/MG), 2) in Cerrado - Malves and FrieiroCosta 2012 (n=1/Ingaí/MG), 3) no biome data Mendonça et al. 2014 ( $\mathrm{n}=14 / \mathrm{RS})$.

Pluchea sagittalis (Lam.) Cabrera (native species, $\mathrm{NE})(\mathrm{n}=1)$

Gall (Fig. 98): on leaf, marginal roll, green, glabrous, and one-chambered. Galler: not determined. Path: Posto Marcão-Abrigo Rebouças, 24/II/2014.

No previous gall records on this plant species. Pentacalia desiderabilis (Vell.) Cuatred. (endemic in Brazil, Cerrado and Atlantic forest, NE) $(n=1)$

Gall (Fig. 99): on stem, globoid, brown, glabrous, and one-chambered. Galler: not determined. Paths: Morro do Couto, 05/I/2015; Travessia Ruy Braga, 15/IX/2015. 
Previous gall records on this plant species: in Atlantic forest - Toma and Mendonça 2013 (N=1/ São Francisco de Paula/RS).

Piptocarpha leprosa (Less.) Baker (native species) $(\mathrm{n}=2)$

Gall (Fig. 100): on midvein, fusiform, green, glabrous, and multichambered. Galler: Cecidomyiidae (02 larvae). Path: BR-485, 23/ II/2015.

Gall (Fig. 101): on leaf, globoid, green, glabrous, and one-chambered. Galler: not determined (empty galls). Path: BR-485, 23/ II/2015.

No previous gall records on this plant species. Piptocarpha macropoda (DC.) Baker (endemic in Brazil, Cerrado and Atlantic forest, NE) $(n=1)$

Gall (no fig.): on leaf vein, globoid, green, glabrous, and one-chambered. Galler: not determined (empty galls). Path: Travessia Ruy Braga, 24/II/2015.

No previous gall records on this plant species. Pterocaulon virgatum (L.) DC. (native species, $\mathrm{NE}$ ) (first record in RJ and Atlantic forest) (NE) $(\mathrm{n}=1)$

Gall (Fig. 102): on stem, globoid, whittish, glabrous, and one-chambered. Galler: not determined. Parasitoids: Hymenoptera (01 larva). Path: Pedra do Altar, 08/IV/2014.

No previous gall records on this plant species. Senecio L. sp. (native genus) $(\mathrm{n}=1)$

Gall (no fig.): on leaf vein, fusiform, yellowish, glabrous, and one-chambered. Galler: not determined. Parasitoids: Hymenoptera (fragments). Path: Água Branca-Ruy Braga, 18/VI/2014.

No previous gall records on this plant genus. Symphyopappus itatiayensis (Hieron.) R. M. King and H. Rod (endemic in Brazil, Cerrado and Atlantic forest, NE) (first record in altitude fields) $(\mathrm{n}=2)$.

Gall (Fig. 103): on leaf, amorphous, green, glabrous, and one-chambered. Galler: Lepidoptera
(04 catterpilars). Paths: Pedra da Maçã, Tartaruga and Assentada, 27/I/2015; Cinco Lagos, 11/V/2015.

Gall (Fig. 104): on bud, ovoid, brown, glabrous, one or multi-chambered. Galler: not determined. Associated fauna: Coleoptera (03 larvae), Hymenoptera (01 pupa), 1 Tephritidae (01 adult). Paths: Cinco Lagos, 11/V/2015, Morro do Couto, 05/10/2015.

No previous gall records on this plant species. Symphyopappus reticulatus Baker (endemic in Brazil, Cerrado, NE) (first record in MG and Atlantic forest $)(n=3)$

Gall (Fig. 105): on apical bud, globoid, with apical and cylindrical projections, brown, and glabrous. Galler: Cecidomyiidae (01 pupa). Path: Travessia Serra Negra, 14-15/X/2014.

Gall (Fig. 106): on lateral bud, globoid, with a single apical projection, brown, glabrous, and onechambered. Galler: Coleoptera (01 larva). Path: Travessia Serra Negra, 14-15/X/2014.

Gall (Fig. 107): on stem and midvein, fusiform, green, glabrous, and one-chambered. Galler: Cecidomyiidae (larval fragments). Inquilines: Muscomorpha cfr. (03 larvae). Parasitoids: Hymenoptera (04 pupae). Path: Travessia Serra Negra, 14-15/X/2014.

Previous gall records on this plant species: 1) in Cerrado - Carneiro et al. 2009b ( $\mathrm{n}=1 /$ Cadeia do Espinhaço/MG), 2) no biome data: - Mendonça et al. 2014 ( $\mathrm{n}=2 / \mathrm{RS})$.

Verbesina glabrata Hook. and Arn. (native species, $\mathrm{NE})(\mathrm{n}=2)$

Gall (Fig. 108): on bud, globoid, whittish, pubescent, and one-chambered. Galler: Cecidomyiidae (nine larvae). Path: Travessia Ruy Braga, 17/III/2015.

Gall (Fig. 109): on leaf, claviform, green, pubescent, and one-chambered. Galler: not determined (empty galls). Path: Travessia Ruy Braga, 17/III/2015.

No previous gall records on this plant species. 


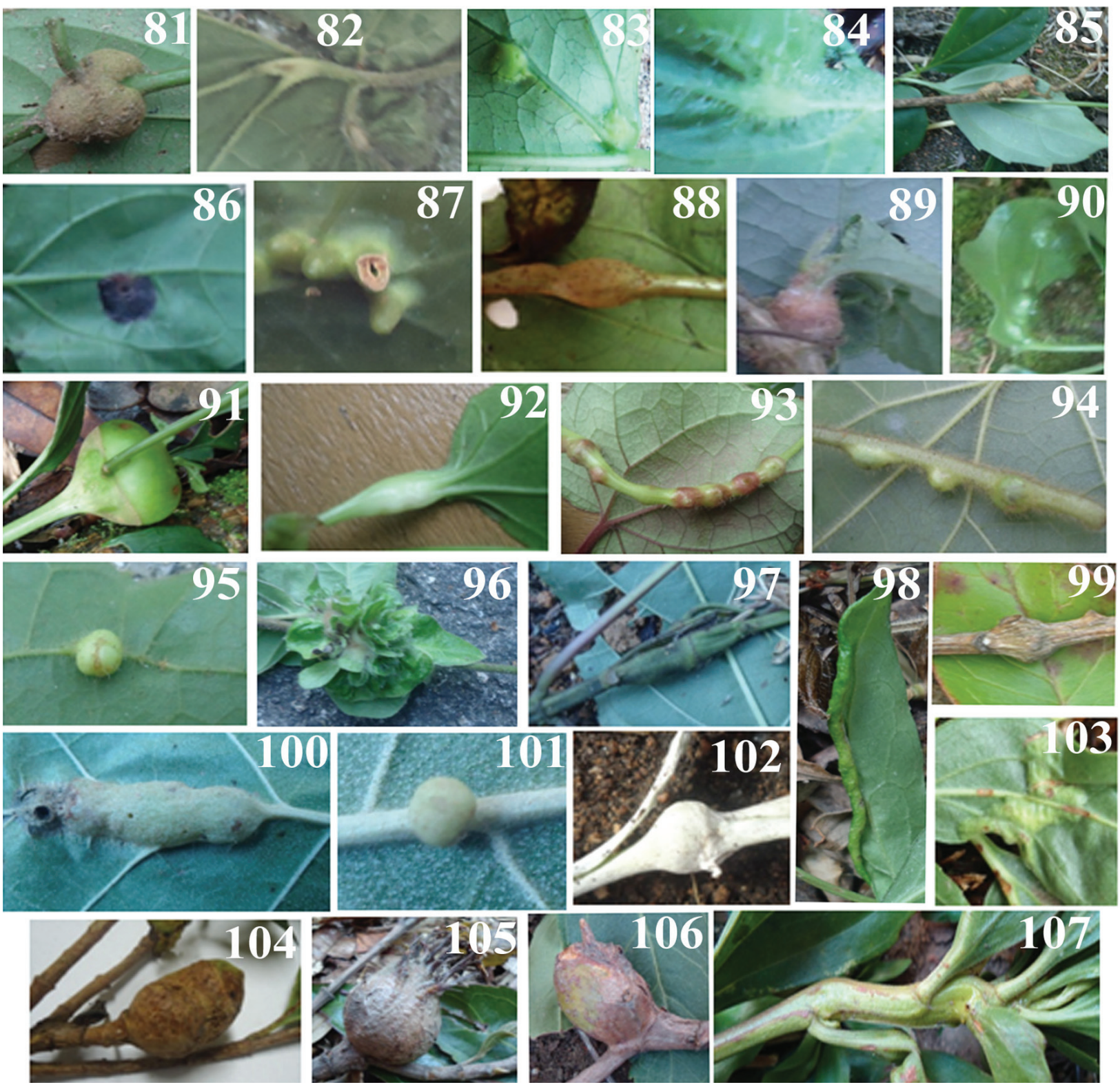

Fig 81-107 - Insect galls of the Parque Nacional do Itatiaia (Southeast Region, Brazil), on Asteraceae, 81, Mikania pseudohoffmanniana, globoid bud gall, 82, M. setigera, fusiform leaf vein gall, 83, Mikania sp.1, globoid leaf gall, 84, Mikania sp.2, fusiform leaf midvein gall, 85-92, Mikania sp.3, 85, fusiform subterraneous root gall, 86, discoid leaf gall, 87, conical leaf gall, 88, fusiform stem gall, 89, fusiform leaf petiole and midvein gall, 90, globoid leaf midvein gall, 91, globoid stem and bud gall, 92, fusiform leaf petiole gall, 93, Mikania sp.4, globoid stem, leaf petiole and vein gall, 94-95, Mikania sp.5, 94, globoid and fusiform, unilateral stem gall, 95, globoid leaf vein gall, 96, Mikania sp.6, rosette bud gall, 97, Mikania sp.7, fusiform stem gall, 98, Pluchea sagittalis, marginal leaf roll, 99, Pentacalia desiderabilis, globoid stem gall, 100-101, Piptocarpha leprosa, 100, fusiform midvein gall, 101, globoid leaf gall, 102, Pterocaulon virgatum, globoid stem gall, 103-104, Symphyopappus itatiayensis, 103, amorphous leaf gall, 104, ovoid bud gall, 105-107, S. reticulatus, 105, globoid apical bud gall,106, globoid lateral bud gall, 107, fusiform stem and leaf midvein gall. 
Vernonanthura membranacea (Gardner) H.Rob. (native species, NE) (first record in RJ and Atlantic Forest) $(\mathrm{n}=1)$

Gall (Fig. 110): on bud, globoid, green, glabrous, and one or three-chambered. Galler: Tephritidae, Diptera (04 puparia, 02 adults). Parasitoids: Hymenoptera (01 adult). Paths: Cachoeira do Aiuruoca, 19/III/2014; Pedra da Maçã, Tartaruga and Assentada, 27/I/2015.

No previous gall records on this plant species. Vernonanthura montevidensis (Spreng.) H.Rob. (native species, NE) (first record in RJ) $(n=1)$

Gall (Fig. 111): on leaf, lineal, yellow, glabrous, and one-chambered. Galler: Lepidoptera (01 catterpilar). Path: Pedra do Altar, 08/IV/2014.

Previous gall records on this plant species: Maia et al. 2014 ( $\mathrm{n}=1 /$ Santa Teresa/SP).

Vernonanthura $\mathrm{H}$. Rob. sp. $(\mathrm{n}=2)$

Gall (Fig. 112): on stem, fusiform, brown, glabrous, and one-chambered. Galler: Tephritidae. Path: BR-485, 23/II/2015.

Gall (Fig. 113): on vein, fusiform, green, glabrous, and one-chambered. Galler: not determined (empty galls). Path: BR-485, 23/ II/2015

No previous gall records on this genus.

Vernonia Schreb. sp. 1 (native genus) (first occurrence in RJ) ( $\mathrm{n}=1)$

Gall (Fig. 114): on stem, fusiform, brown, glabrous, and one-chambered. Galler: Lepidoptera (01 catterpilar). Paths: Água Branca-Ruy Braga, 18/VI/2014, Viúva Hansen, 10/IX/2014.

Vernonia Schreb. sp. 2 ( $\mathrm{n}=1)$

Gall (Fig. 115): on stem, fusiform, brown, glabrous, and one-chambered. Galler: Lepidoptera. Successors: ants (Formicidae). Paths: Travessia Ruy Braga, 14/IV/2015, 15/IX/201; Travessia Serra Negra, 14-15/X/2014; Três Picos, 11/XI/2014.

Vernonia Schreb. sp.3 $(\mathrm{n}=1)$

Gall (Fig. 116): on stem, leaf petiole and midvein, globoid or fusiform, green, with trichomes, and one-chambered. Galler: Cecidomyiidae. Path: BR-485, 28/01/2015.

Previous gall records on undetermined species of Vernonia: 1) in Atlantic forest - Maia et al. 2014 ( $n=1 /$ Santa Teresa/ES), Maia 2013b ( $n=2 /$ São Tomé das Letras/MG), Maia 2014 (n=4/Itamonte/ MG), 2) in Cerrado - Maia and Fernandes 2004 ( $\mathrm{n}=1 /$ Serra de São José/MG), Gonçalves-Alvim and Fernandes $2001(\mathrm{n}=1 / \mathrm{MG})$

\section{BERBERIDACEAE $(\mathrm{N}=1)$}

Berberis laurina Billb. (native species, Atlantic forest, $\mathrm{NE})(\mathrm{n}=1)$

Gall (Fig. 117): on leaf, globoid, red, glabrous, and one-chambered. Galler: Psyllidae (Hemiptera). Path: Pedra do Camelo, 14/IX/2015.

Previous gall records on this plant species: no biome data - Mendonça et al. 2014 ( $n=2 / R S)$.

\section{BIGNONIACEAE $(\mathrm{N}=8)$}

Fridericia Mart. sp. (native genus) $(\mathrm{n}=1)$

Gall (Fig. 118): on stem and leaf vein, fusiform, green, glabrous, and one-chambered. Galler: not determined. Parasitoids: Hymenoptera (02 pupae, 01 adult). Paths: Lago Azul 18/III/ 2014, BR-485, 28/I/2015.

Previous gall records on this plant genus: as Arrabidaea (sin. het.):1) in Cerrado - Santos et al. 2010 ( $\mathrm{n}=2 /$ Goiânia/GO), Araújo et al. 2011 ( $\mathrm{n}=1 /$ Serra dos Pireneus/GO), Gonçalves-Alvim and Fernandes 2001 ( $\mathrm{n}=1 / \mathrm{MG})$, Saito and UrsoGuimarães 2012 (n=4/Jataí/SP), 2) in Amazonian forest - Almada and Fernandes $2011 \quad(n=1 /$ Oriximiná/PA), Maia $2011(\mathrm{n}=3$ / Oriximiná/PA); 3 ) in Atlantic forest: as Fridericia sp. - Fernandes et al. 2001 ( $\mathrm{n}=5 /$ Vale do Rio Doce/MG).

Dolichandra unguis-cati (L.) L.G.Lohmann (native species, NE) $(\mathrm{n}=2)$

Gall (Fig. 119): on stem, fusiform, green, glabrous, and multichambered. Galler: Muscomorpha (19 puparia). Paths: Cachoeira 
Itaporani, 17 /III/2014, 12/V/2014; Três Picos, IX/2014; Travessia Ruy Braga, 10-12/XI/2014, 08/ XII/2014, 16/III/2015; BR-485, 12/XI/2014.

Gall (Fig. 120): on bud and stem, globoid, brown, glabrous, and multichambered. Galler: not determined (empty galls). Path: BR-485, 12/ $\mathrm{XI} / 2014$.

No previous gall records on this plant species. Jacaranda copaia (Aubl.) D. Don (native species, $\mathrm{NE}$ ) (first record in Southeast Region and Atlantic forest $)(n=1)$

Gall (Fig. 121): on stem and leaf petiole, fusiform, brown, and glabrous. Galler: not determined (empty galls). Path: Travessia Ruy Braga, 10-12/XI/2014.

No previous gall records on this plant species. Mansoa difficilis (Cham.) Bureau and K.Schum. (native species, NE) $(\mathrm{n}=1)$

Gall (Fig. 122): on stem, fusiform, brown, glabrous, and one-chambered. Galler: Cecidomyiidae (02 larvae). Path: BR-485, 28/01/2015.

No previous gall records on this plant species. Mansoa DC.sp. (native genus) $(\mathrm{n}=1)$

Gall (Fig. 123): on stem, fusiform, brown, glabrous, and one-chambered. Galler: not determined. Path: Travessia Ruy Braga, 08/ VII/2014.

No previous gall records on this plant genus.

Not determined $1(n=1)$

Gall (Fig. 124): on bud, fusiform, reddish, glabrous, and one-chambered. Galler: not determined. Path: Agulhas Negras, 08/VII/2014.

Not determined $2(n=1)$

Gall(Fig. 125): on leaf, discoid, green, glabrous, and one-chambered. Galler: not determined. Path: Casa 25, 08/XII/2015.

BORAGINACEAE $(\mathrm{N}=1)$

Cordia L. sp. (native genus) $(\mathrm{n}=1)$
Gall (Fig. 126): on stem, fusiform, brown, pubescent, and one-chambered. Galler: not determined. Parasitoids: Hymenoptera (fragments). Path: Centro de Visitantes, 07-08/VII/2014.

Previous gall records on undetermined species of Cordia: 1) in Cerrado - Araújo et al. 2012 ( $\mathrm{n}=1 /$ GO), Maia and Fernandes 2004 (n=1/Serra de São José/MG), 2) Caatinga-Cerrado - Costa et al. 2015 (n=1/Caetité/BA), 3) in Amazonian forest Almada and Fernandes 2011 ( $\mathrm{n}=2 /$ Oriximiná/PA), Maia 2011 ( $\mathrm{n}=1 /$ Oriximiná/PA).

\section{BURSERACEAE $(\mathrm{N}=4)$}

Protium crassipetalum Cuatrec. (native species, NE) (first record in Southeast Region and Atlantic forest $)(n=1)$

Gall (Fig. 127): on leaf, globoid, opened, green, glabrous, and one-chambered. Galler: Hemiptera. Path: Ecoarte-Lago Azul, 08/IV/2014.

No previous gall records on this plant species. Protium tenuifolium (Engl.) Engl. (native species, NE) (first record in Southeast Region and Atlantic forest $)(n=1)$

Gall (Fig. 128): on leaf, discoid, opened, green, glabrous, and one-chambered. Galler: Hemiptera. Path: Ecoarte-Lago Azul, 08/IV/2014.

Previous gall records on this plant species: in Amazonian forest - Almada and Fernandes 2011 ( $\mathrm{n}=1 /$ Oriximiná/PA).

Protium Burm. f. sp. (native genus) $(\mathrm{n}=2)$

Gall (Fig. 129): on leaf, marginal roll, green, glabrous, one-chambered. Galler: Cecidomyiidae. Paths: Viúva Hansen, 10/IX/2014; Barbosa Rodrigues (obelisk), 10/XII/2014; BR-485, 23/ II/2015.

Gall (Fig. 130): on leaf, hemispherical, green, glabrous, and one-chambered. Galler: Hemiptera. Paths: Viúva Hansen, 10/IX/2014; BR-485, 23/ II/2015.

Previous gall records on undetermined species of Protium: 1) in Amazonian forest - Almada and 
Fernandes 2011 ( $\mathrm{n}=5 /$ Oriximiná/PA), Araújo et al. 2012 ( $n=4 /$ Oriximiná/PA), 2) in Cerrado - Araújo et al. $2015(n=3 / G O)$.

\section{CANNABACEAE $(\mathrm{N}=3)$}

Celtis iguanaea (Jacq.) Sarg. (native species, NE) $(\mathrm{n}=1)$

Gall (Fig. 131): on leaf vein, fusiform, green, with trichomes, and one-chambered. Galler: Cecidomyiidae (03 larvae). Path: Casa 25, 08/ $\mathrm{XII} / 2015$.

Previous gall records on this plant species: 1) in Cerrado - Coelho et al. 2009 ( $n=1 /$ Serra do Cipó/MG), Santos et al. 2010 ( $\mathrm{n}=1 /$ Goiânia/GO), 2) no biome data - Mendonça et al. 2014 ( $n=3 / R S)$.

Celtis L. sp. (native genus) $(\mathrm{n}=2)$

Gall (Fig. 132): on stem, fusiform, green, with brow micropubescence. Galler: not determined. Path: Travessia Ruy Braga, 04/VIII/2014.

Gall (no fig.): on leaf petiole, globoid, brown, glabrous, and one-chambered. Galler: Lepidoptera (01 catterpilar). Path: Travessia Ruy Braga, 04/ VIII/2014.

Previous gall records on undetermined species of Celtis: in Atlantic forest-Fernandes and Negreiros 2006 (n=1/Aimorés/MG).

\section{COMBRETACEAE $(\mathrm{N}=2)$}

Combretum fruticosum (Loefl.) Stuntz (native species, NE) (first record in altitude fields) $(n=1)$

Gall (Fig. 133): on leaf midvein, fusiform, green, glabrous, and one-chambered. Galler: not determined (empty gall). Path: Prateleiras, 09/ IV/2014.

No previous gall records on this plant species. Combretum Loefl. sp. (native genus) $(\mathrm{n}=1)$

Gall (Fig. 134): on leaf, globoid, green, glabrous, and one-chambered. Galler: not determined. Path: Centro de Visitantes, 07-08/ VII/2014.
Previous gall records on Combretum: 1) in Caatinga: on C.leprosum Mart. - Tavares 1925 (n=1/Ceará), 2) in Amazonian forest on $C$. laxum Jacq. - Almada and Fernandes 2011 ( $\mathrm{n}=1$ / Oriximiná/Pará).

\section{CONVOLVULACEAE $(\mathrm{N}=5)$}

Dicranostyles Benth. sp. (native genus) $(\mathrm{n}=2)$

Gall (Fig. 135): on stem, fusiform, green, glabrous, and one or multichambered. Galler: Cecidomyiidae (01 pupa, 01 pupal exuvia, 03 adults). Parasitoids: Hymenoptera (01 pupa). Paths: Cachoeira Itaporani, 17/III/2014; Donati-Simon 2, 08/IX/2014; Três Picos, 08/IX/2014; Travessia Ruy Braga, 16/III/2015.

Gall (no fig.): on leaf, discoid, green, and glabrous. Galler: not determined (empty galls). Path: Cachoeira Itaporani, 17 /III/2014, 12/V/2014.

No previous records on this plant genus.

Ipomoea L. sp1. (native genus) $(\mathrm{n}=2)$

Gall (Fig. 136): on leaf, discoid, green, glabrous, and one-chambered. Galler: not determined. Path: Hotel Donati, 04/VIII/2014.

Gall(nofig.): on stem, globoid, brown, glabrous, and one-chambered. Galler: Cecidomyiidae (03 larvae). Path: Hotel Donati, 04/VIII/2014.

Ipomoea L. $\mathrm{sp} 2 .(\mathrm{n}=1)$

Gall (Fig. 137): on stem, globoid, brown, glabrous, and one-chambered. Galler: Cecidomyiidae (03 larvae). Path: Hotel Donati, 04/ VIII/2014.

No previous records on this plant genus.

\section{CUNONACEAE $(\mathrm{N}=2)$}

Lamanonia ternata Vell. (endemic in Brazil, Cerrado and Atlantic forest, NE) (first record in altitude fields) $(\mathrm{n}=1)$

Gall (no fig.): on leaf petiole, fusiform, brown, glabrous, and one-chambered. Galler: not determined. Path: Travessia Ruy Braga, 14/ IV/2015. 

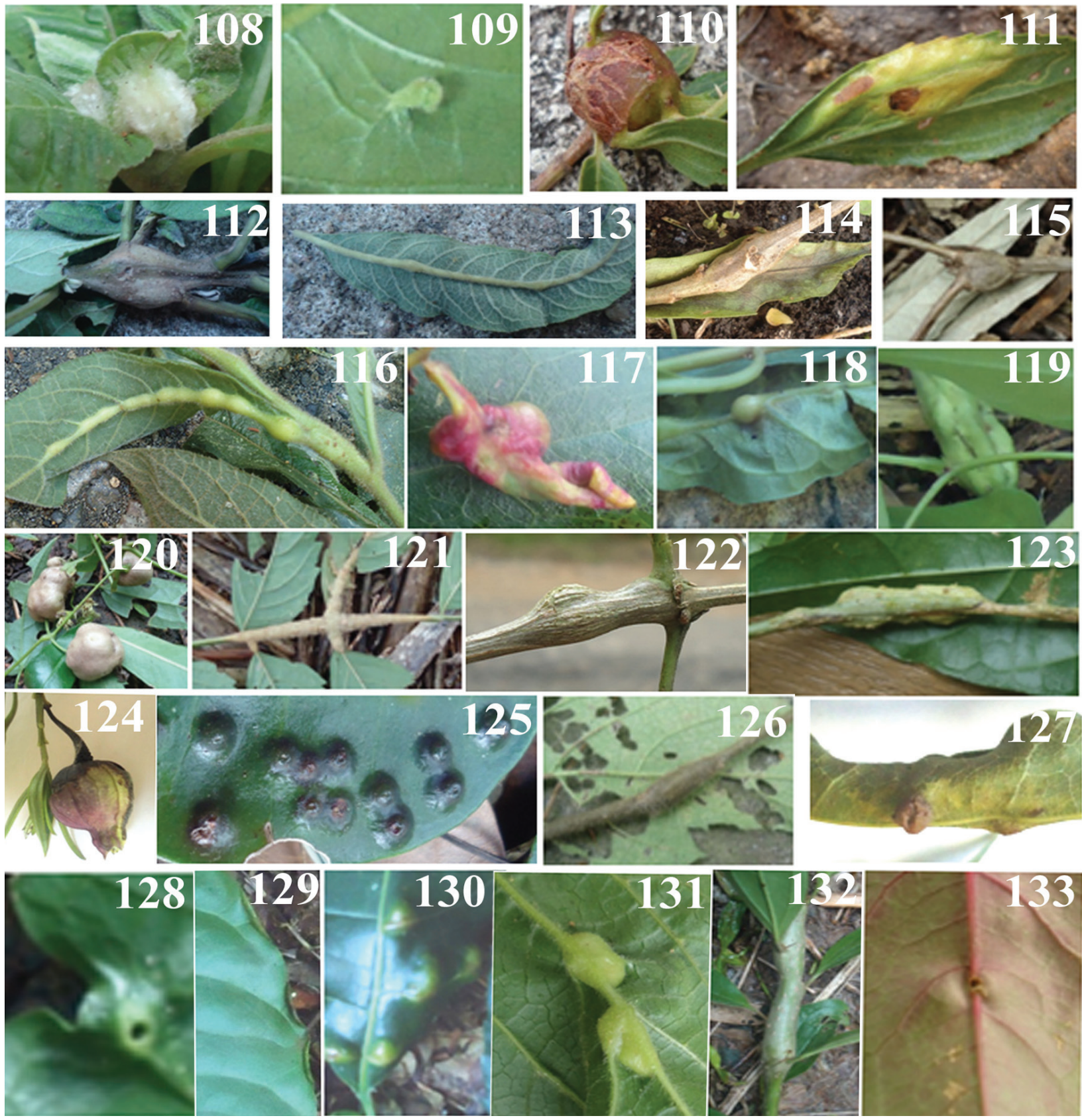

Figure 108-133 - Insect galls of the Parque Nacional do Itatiaia (Southeast Region, Brazil), 108-109, Verbesina glabrata, 108, globoid bud gall, 109, claviform leaf gall, 110, Vernonanthura menbranacea, globoid bud gall, 111, V. montevidensis, lineal leaf gall, 112-113, Vernonanthura sp., 112, fusiform stem gall, 113, fusiform vein gall, 114, Vernonia sp.1, fusiform stem gall, 115, Vernonia sp.2, fusiform stem gall, 116, Vernonia sp.3, leaf petiole and midvein, globoid or fusiform, 117, on Berberidaceae, Berberis laurina, globoid leaf gall, 118-125, on Bignoniaceae, 118, Fridericia sp., fusiform stem and leaf vein gall, 119-120, Dolichandra unguis-cati, 119, fusiform stem gall, 210, globoid bud and stem gall, 121, Jacaranda copaia, fusiform stem and leaf petiole gall, 122, Mansoa difficilis, fusiform stem gall, 123, Mansoa sp., fusiform stem gall, 124, Bignoniaceae sp.1, fusiform bud gall, 125, Bignoniaceae sp.2, discoid leaf gall, 126, on Boraginaceae, Cordia sp., fusiform stem gall, 127-130, on Burseraceae, 127, Protium crassipetalum, globoid leaf gall, 128, P. tenuifolium, discoid leaf gall, 129-130, Protium sp., 129, marginal leaf roll, 130, hemispherical leaf gall, 131-132, on Cannabaceae, 131, Celtis iguana, fusiform leaf vein gall, 132, Celtis sp., fusiform stem gall, 133, on Combretaceae, Combretum fruticosum, fusiform leaf midvein gall. 
No previous gall records on this plant genus. Weinmannia humilis Engl. (endemic in Brazil, Atlantic forest, NE) (first record in altitude fields) $(\mathrm{n}=1)$

Gall (Fig. 138): on bud, globoid, green, micropubescent, and one-chambered. Galler: Hemiptera. Paths: Prateleiras, 09/IV/2014, Asa de Hermes, 16/IX/2015.

No previous gall records on this plant species.

\section{CURCUBITACEAE $(\mathrm{N}=1)$}

Cayaponia pilosa (Vell.) Cogn. (native species, $\mathrm{NE})(\mathrm{n}=1)$

Gall (Fig. 139): on bud, cylindrical, green, with five green filiform projections, scattered trichomes, and one-chambered. Galler: Lepidoptera (02 catterpilars). Path: BR-485, 26/I/2015.

No previous gall records on this plant species. Previous records on this plant genus: 1) in Atlantic forest: on Cayaponia sp. - Toma and Mendonça 2013 ( $\mathrm{n}=2 / \mathrm{SFP} / \mathrm{RS})$.

\section{DILLENIACEAE $(\mathrm{N}=4)$}

Davilla Vand. sp. (native genus) $(\mathrm{n}=1)$

Gall (Fig. 140): on bud, pineapple-like, green, glabrous, and imbricated. Galler: Lepidoptera. Paths: Travessia Ruy Braga, 24/II/2015; BR-485, 23/II/2015.

Previous records of the same gall morphotype: 1) in Atlantic forest - on Davilla sp.: Maia et al. 2014 (Santa Teresa/ES), 2) in Cerrado - on Davilla braziliana DC: Maia and Fernandes 2004 (Serra de São José/MG).

Doliocarpus Rol. sp.1 (native genus) ( $\mathrm{n}=1$ )

Gall (Fig. 141): on vein, fusiform, yellowish, pubescent, and one-chambered. Galler: not determined (empty galls). Path: Hotel Donati, 04/ VIII/2014.

Doliocarpus Rol. sp.2 ( $\mathrm{n}=2)$

Gall (Fig. 142): on bud, globoid, brown, glabrous, and one-chambered. Galler: not determined. Path: Travessia Serra Negra, 14-15/ $\mathrm{X} / 2014$.

Gall (Fig. 143): on stem, fusiform, brown, glabrous, and multichambered. Galler: probably Hymenoptera (03 larvae). Path: Travessia Ruy Braga, 14/IV/2015.

Previous gall records on this plant genus: in Cerrado - Maia et al. 2010 ( $\mathrm{n}=1$ / Dores do Indaiá/ $\mathrm{MG})$.

\section{DIOSCOREACEAE $(\mathrm{N}=3)$}

Dioscorea L. sp. (native genus) $(\mathrm{n}=3$ )

Gall (Fig. 144): on leaf blade and petiole, globoid, green, glabrous, and one-chambered. Galler: not determined (empty galls). Path: Centro de Visitantes, 07-08/VII/2014.

Gall (Fig. 145): on leaf, cylindrical, green, glabrous, and one-chambered. Galler: not determined. Successors: Thysanoptera (3 nymphs). Path: Centro de Visitantes, 07-08/VII/2014.

Gall (Fig. 146): on leaf vein, fusiform, greenish, glabrous, and one-chambered. Galler: not determined (empty galls). Path: Centro de Visitantes, 07-08/VII/2014.

Previous gall records on this plant genus: 1) in Atlantic forest - Santos et al. 2013 ( $n=1 / P E), 2)$ no biome data - Mendonça et al. 2014 ( $n=2 / R S)$.

\section{EBENACEAE $(\mathrm{N}=1)$}

Diospyros L. sp. (native genus) $(\mathrm{n}=1)$

Gall (Fig. 147): on bud, globoid, green, with red pubescence, and one-chambered. Galler: Lepidoptera (pupa fragments). Path: Três Picos, 11/XI/2014.

Previous gall records on this plant genus: in Atlantic forest - Maia 2013b ( $n=1 /$ São Tomé das Letras/MG).

EUPHORBIACEAE $(\mathrm{N}=14)$

Acalypha communis Müll. Arg. (native species, $\mathrm{NE})(\mathrm{n}=1)$ 
Gall (Fig. 148): on leaf, conical, green, hairy, and one-chambered. Galler: not determined. Path: BR-485, 26/I/2015.

Previous gall records on this plant genus: 1) in Atlantic forest: on Acalypha sp.- Tavares 1918 $(\mathrm{n}=1 / \mathrm{RJ}), 2)$ in Pantanal: on Acalypha cf. communis - Julião et al. 2002 (n=1/MS).

Croton campanulatus Caruso and Cordeiro (endemic in Brazil, Atlantic forest, NE) (n=1)

Gall (Fig. 149): on stem, fusiform, brown, glabrous, and one-chambered. Galler: not determined. Parasitoids: Hymenoptera (01 adult). Path: BR485, 26/I/2015.

No previous gall records on this plant species. Croton floribundus Spreng. (endemic in Brazil, Caatinga, Cerrado and Atlantic forest, NE) $(n=4)$

Gall (Fig. 150): on leaf, globoid, green or yellow, hairy, and one-chambered. Galler: Cecidomyiidae (02 larvae, 13 pupae). Parasitoids: Hymenoptera (08 larvae, 03 pupae, 01 adult). Inquilines: Lepidoptera (02 catterpilars). Paths: Lago Azul, 18/III/ 2014; Ecoarte-Lago Azul, 08/ IV/2014; Centro de Visitantes, 07-08/VII/2014; Casa 16, 07/VII/2014; BR-485, 04/VIII/2014, 09/XII/2014，26/I/2015，23/II/2015; Barbosa Rodrigues-Mirante do Último Adeus, 09/XII/2014, Barbosa Rodrigues (obelisk), 10/XII/2014.

Gall(Fig. 151): on leaf, discoid, green, glabrous, and one-chambered. Galler: Cecidomyiidae (02 larvae). Paths: Lago Azul, 18/III/ 2014; EcoarteLago Azul, 08/IV/2014; BR-485, 23/II/2015.

Gall (Fig. 152): on stem, fusiform, brown, glabrous, and multichambered. Galler: not determined (empty gall). Path: BR-485, 23/II/2015.

Gall (Fig. 153): on leaf, marginal roll, green, glabrous, and one-chambered. Galler: not determined (empty galls). Path: BR-485, 04/ VIII/2014.

Previous gall records on this plant species: 1) in Atlantic forest - Maia et al. $2014(n=1 /$ Santa Teresa/ES), Rodrigues et al. 2014 ( $\mathrm{n}=3 / \mathrm{Ilha}$ da Marambaia/RJ), 2) in Cerrado - Urso-Guimarães et al. 2003 ( $\mathrm{n}=1 /$ Delfinópolis/MG), Maia and Fernandes 2004 (n=5/Serra de São José/MG)

Croton L. sp. 1 (native genus) $(\mathrm{n}=1)$

Gall (Fig. 154): on stem, globoid, brown, and glabrous. Galler: not determined. Path: Travessia Serra Negra, 14-15/X/2014.

Previous gall records on undetermined species of Croton L.: 1) in Atlantic forest - Rübsaamen 1905 ( $\mathrm{n}=2 /$ Itabininha/RJ), Fernandes et al. 2001 ( $n=1 /$ Vale do Rio Doce/MG), Maia 2013b (n=2/São Tomé das Letras/ES), Maia 2014 (n=5/Itamonte/ MG), Maia et al. 2014 ( $n=1 /$ Santa Teresa/ES), Toma and Mendonça 2013 (n=1/São Francisco de Paula/RS), 2) in Cerrado - Malves and FrieiroCosta 2012 ( $\mathrm{n}=4 /$ Ingaí/MG)

Dalechampia scandens L. (native species, NE) (first record in $\mathrm{RJ})(\mathrm{n}=1)$

Gall (Fig. 155): on leaf and bud, globoid, yellow, with white trichomes, and one-chambered. Galler: not determined. Parasitoids: Hymenoptera (02 larvae, 05 pupae, 01 adult). Path: Viúva Hansen, 10/IX/2014.

No previous gall records on this plant species. Manihot esculenta Crantz (native species) (first record in RJ and Atlantic forest, NE) $(n=1)$

Gall (Fig. 156): on stem, globoid, brown, and glabrous. Galler: not determined. Path: DonatiSimon 2, 08/IX/2014.

Previous gall records on this plant species: as Manihot utilissima Pohl (sin. het), in Atlantic forest - Rübsaamen 1908 (n=1/RJ).

Pachystroma longifolium (Nees) Im. Johnst (native species, NE) $(\mathrm{n}=1)$

Gall (Fig. 157): on leaf, conical, green, glabrous, and one-chambered. Galler: Hemiptera (01 nymph). Path: BR-485, 26/I/2015.

Previous gall records on this plant species: 1) in Atlantic forest - Maia and Carvalho-Fernandes 2016 (n=3/São Francisco de Itabapoana/RJ).

Sapium Jacq. sp.1 (native genus) $(\mathrm{n}=2)$

Gall (Fig. 158): on leaf blade and petiole, globoid, green, glabrous, and one-chambered. 
Galler: Hemiptera. Path: Hotel Donati, 04/ VIII/2014.

Gall (Fig. 159): on bud, conical, wide, brown, glabrous, and one-chambered. Galler: Lepidoptera. Path: Hotel Donati, 04/VIII/2014.

Sapium Jacq. sp.2 ( $\mathrm{n}=1)$

Gall (Fig. 160): on leaf vein, globoid, green, glabrous, and one-chambered. Galler: Coccoidea (Hemiptera). Path: Travessia Ruy Braga, 10-12/ $\mathrm{XI} / 2014$.

Previous gall records on this plant genus: 1) no biome data: on Sapium glandulosum (L.) Morong ( $\mathrm{n}=1 / \mathrm{RS}$ ) and on $S$. haematospermum Mull. Arg. $(\mathrm{n}=1 / \mathrm{RS})$ - Mendonça et al. 2014

Sebastiania Spreng. sp. (native genus) $(\mathrm{n}=1)$

Gall (Fig. 161): on leaf midvein, globoid, brownish, glabrous, and one-chambered. Galler: Cecidomyiidae (01 larva). Path: Água Branca-Ruy Braga, 18/VI/2014.

Previous gall records on this plant genus: 1) in Cerrado - Araújo et al. 2011 (n=1/Pireneus/GO), 2) no biome data: on Sebastiania brasiliensis Spreng. ( $n=1 / \mathrm{RS}$ ) and on S. commersonianna (Baill.) L.B.Sm. and Downs ( $\mathrm{n}=1 / \mathrm{RS})$ - Mendonça et al. 2014.

FABACEAE $(\mathrm{N}=29)$

Calliandra brevipes Benth (native species, NE) $(\mathrm{n}=1)$

Gall (Fig. 162): on bud, ovoid, brown, glabrous, and multichambered. Galler: Hymenoptera (16 adults). Path: Casa 25, 08/XII/2015.

Previous gall records on this plant: no biome data - Mendonça et al. 2014 ( $n=2 / R S)$.

Centrosema sp. (DC.) Benth. (native genus) (n=1)

Gall (Fig. 163): on stem, fusiform, brown or reddish, and glabrous. Galler: not determined. Path: Travessia Ruy Braga, 15/IX/2015.

Previous gall records on this plant genus: 1) in Atlantic forest: on C. virginianum (L.) Benth Carvalho-Fernandes et al. 2016 ( $n=1 / R L / R J)$.
Dahlstedtia pinnata (Benth.) Malme (endemic in Brazil, Atlantic forest, NE) ( $\mathrm{n}=1)$

Gall (Fig. 164): on stem, fusiform, green, glabrous, and one-chambered. Galler: not determined. Sucessors: Sciaridae (06 adults, 04 pupae, 05 pupal exuviae and 06 larvae). Path: BR$485,28 / \mathrm{I} / 2015$.

No previous gall records on this plant species. Dalbergia foliosa (Benth.) A.M. Carvalho (native species, NE) (first record in Southeast region and Atlantic forest $)(n=1)$

Gall (Fig. 165): on leaf, marginal roll, green, glabrous, and one-chambered. Galler: not determined (empty galls). Path: Travessia Ruy Braga, 24/II/2015.

Previous gall records on this plant species: 1) in Atlantic forest - Maia 2013b (n=3/STL/MG).

Dalbergia subcymosa Ducke (native species, NE) (first record in Southeast Region and Atlantic forest $)(n=1)$

Gall (Fig. 166): on leaf, coalescent, green, glabrous, and one-chambered. Galler: not determined (empty galls). Path: Três Picos, 11/ $\mathrm{XI} / 2014$.

No previous gall records on this plant species. Derris Lour. sp. (native genus $(\mathrm{n}=2$ )

Gall (Fig. 167): on leaf, fold, green, glabrous, and one-chambered. Galler: not determined. Path: Travessia Ruy Braga, 08/VII/2014.

Gall (Fig. 168): on leaf vein, fusiform, brown, glabrous, and one-chambered. Galler: not determined (egg). Path: Travessia Ruy Braga, 08/ $\mathrm{VII} / 2014$.

Previous gall records on this plant genus: 1) in Atlantic forest - Santos et al. 2011b: on Derris guilleminiana (Tul.) J.F. Macbr ( $\mathrm{n}=1 / \mathrm{six}$ municipalities/PE).

Grazielia gaudichaudeana (DC.) R.M.King and H.Rob. (endemic in Brazil, Cerrado and Atlantic forest, NE) $(n=3)$

Gall (Fig. 169): on stem, fusiform, brown, and glabrous. Galler: Lepidoptera. Inquilines: 

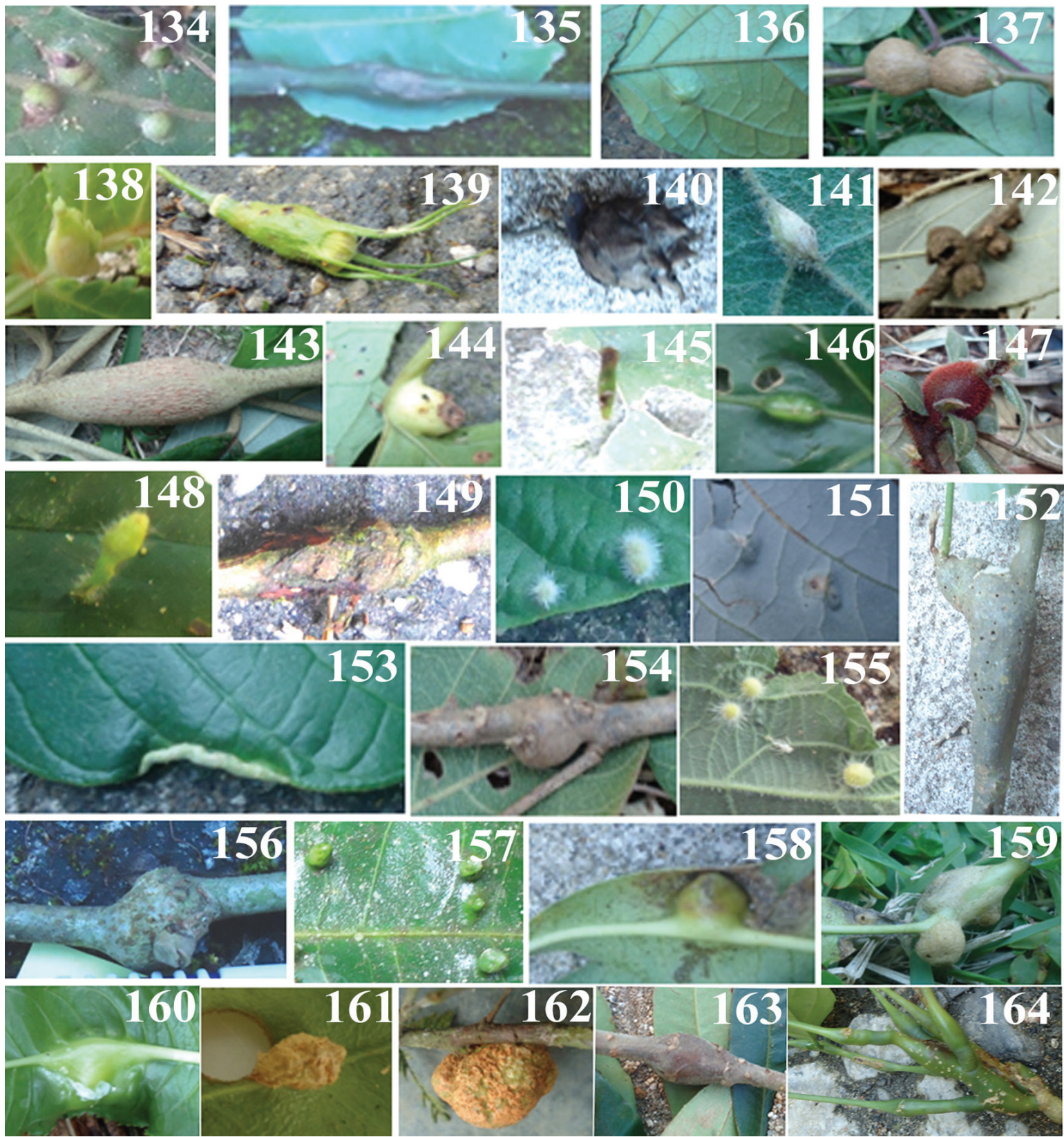

Figure 134-164 - Insect galls of the Parque Nacional do Itatiaia (Southeast Region, Brazil), 134, on Combretaceae, Crombretum sp., globoid leaf gall, 135-137, on Convolvulaceae, 135, Dicranostyles sp., fusiform stem gall, 136, Ipomoea sp.1, discoid leaf gall, 137, Ipomoea sp.2, fusiform stem gall, 138, on Cunonaceae, Weinmannia humilis, globoid bud gall, 139, on Curcubitaceae, Cayaponia pilosa, cylindrical bud gall, 140-143, on Dilleniaceae, 140, Davilla sp., pineapple-like bud gall, 141, Doliocarpus sp.1, fusiform leaf vein gall, 142-143, Doliocarpus sp.2, 142, globoid bud gall, 143, fusiform stem gall, 144-146, on Dioscoreaceae, Dioscorea sp., 144, globoid leaf blade and petiole gall, 145, cylindrical leaf gall, 146, fusiform leaf vein gall, 147, on Ebenaceae, Diospyros sp., globoid bud gall, 148-161, on Euphorbiaceae, 148, Acalypha communis, conical leaf gall, 149, Croton campanulatus, fusiform stem gall, 150-153, C. floribundus, 150, globoid leaf gall, 151, discoid leaf gall, 152, fusiform stem gall, 153, marginal leaf roll, 154, Croton sp., globoid stem gall, 155, Dalechampia scandens, globoid leaf and bud gall, 156, Manihot esculenta, globoid stem gall, 157, Pachystroma longifolium, conical leaf gall, 158-159, Sapium sp.1, 158, globoid leaf blade and petiole gall, 159, conical bud gall, 160, Sapium sp.2, globoid leaf vein gall, 161, Sebastiania sp., globoid leaf midvein gall, 162-164, on Fabaceae, 162, Calliandra brevipes, ovoid bud gall, 163, Centrosema sp., fusiform stem gall, 164, Dahlstedtia pinnata, fusiform stem gall. 
Coleoptera (01 larva). Paths: Pedra da Maçã, Tartaruga and Assentada, 27/I/2015, Cinco Lagos, 12/V/2015, Travessia Ruy Braga, 15/IX/2015.

Gall (no fig.): on leaf, fold, green, glabrous, and one-chambered. Galler: Lepidoptera (03 catterpilars). Path: Travessia Ruy Braga, 13/V/2015.

Gall (Fig. 170): on bud, rosette, green, glabrous, and one-chambered. Galler: not determined (empty galls). Paths: Pedra da Maçã, Tartaruga and Assentada, 27/I/2015, Cinco Lagos, 12/V/2015.

No previous gall records on this plant species. Inga barbata Benth (endemic in Brazil, Cerrado and Atlantic forest, NE) $(n=1)$

Gall (Fig. 171): on leaf, globoid, green, with red trichomes, and one-chambered. Galler: not determined (empty galls). Path: Barbosa Rodrigues (obelisk), 10/X/2014, 10/XII/2014.

No previous gall records on this plant species. Inga gracilifolia Ducke (native species, NE) (first record in Southeast Region and Atlantic forest) $(\mathrm{n}=1)$

Gall (Fig. 172): on apical and lateral bud, globoid, whittish, glabrous, and multichambered (Fig.). Galler: not determined. Parasitoids: Hymenoptera (01 larva/chamber). Path: Três Picos, 08/IX/2014.

Previous gall records on this plant species: in Amazonian forest - Almada and Fernandes 2011 ( $\mathrm{n}=1 /$ Oriximiná/PA).

Inga grandiflora Ducke (native species, NE) (first record in Southeast Region and Atlantic forest) $(\mathrm{n}=3)$

Gall (Fig. 173): on stem, leaf petiole and vein, fusiform, brown, and glabrous. Galler: Cecidomyiidae (12 larvae, 06 pupal exuviae, 02 males) Parasitoids: Hymenoptera (03 larvae, 05 pupae, 06 adults). Inquilines: Thysanoptera (01 adult). Paths: Cachoeira Poranga, 17/III/2014, 07/IV/2014; Três Picos, 13/V/2014, 11/XI/2014, 28/I/2015; Travessia Ruy Braga, 14/V/2014, 08/ VII/2014, 24/II/2015, 16/III/2015; Água BrancaRuy Braga, 18/VI/2014; Donati-Simon 2, 08/
IX/2014; Viúva Hansen, 10/IX/2014; BR-485, 12/ XI/2014; Cachoeira do Pitu, 25/II/2015; Travessia Ruy Braga, 24/II/2015.

Gall (Fig. 174): on leaf, marginal roll, green, glabrous, and one-chambered. Galler: not determined (empty galls). Paths: Viúva Hansen, 10/ IX/2014; Travessia Ruy Braga, 08/XII/2014, 16/ III/2015.

Gall (no fig.): on leaf, globoid, hairy, brown, and one-chambered. Galler: Cecidomyiidae (01 larva). Parasitoids: Hymenoptera (03 larvae, 02 adults). Path: Donati-Simon 2, 08/IX/2014.

No previous gall records on this plant species. Inga marginata Willd. (native species, NE) $(\mathrm{n}=2)$

Gall (Fig. 175): on leaf midvein, fusiform, yellow, glabrous, and one-chambered. Galler: Cecidomyiidae (01 larva). Path: Centro de Visitantes, 07-08/VII/2014.

Gall (Fig. 176): on leaf, globoid, green, glabrous, and one-chambered. Galler: not determined. Path: BR-485, 04/VIII/2014.

Previous gall records on this plant species: 1) in Cerrado - Araújo et al. 2015 ( $\mathrm{n}=1 / \mathrm{GO}), 2)$ no biome data - Mendonça et al. 2014 ( $\mathrm{n}=2 / \mathrm{RS})$.

Inga cf. sessilis (Vell) Mart. (endemic in Brazil, Cerrado, Amazonian and Atlantic forest, NE) $(n=1)$

Gall (Fig. 177): on leaf midvein, fusiform, brown, with brown pubescence, and onechambered. Galler: Cecidomyiidae (01 young larva). Paths: Água Branca-Ruy Braga, 18/ VI/2014; Travessia Ruy Braga, 18/VI/2014.

No previous gall records on this plant species. Luetzelburgia auriculata (Allemão) Ducke (endemic in Brazil, Cerrado, Amazonian and Atlantic forest, NE) (first record in RJ and Atlantic forest $)(n=1)$

Gall (Fig. 178): on bud, globoid, green, glabrous, and one-chambered. Galler: not determined (empty galls). Path: Travessia Ruy Braga, 08/VII/2014.

No previous gall records on this plant species. 
Machaerium nyctitans (Vell.) Benth. (native species, LC) $(\mathrm{n}=2)$

Gall (Fig. 179): on stem, unilateral, globoid, brown, glabrous, and one-chambered. Galler: Lepidoptera (silk in the internal chamber). Paths: Viúva Hansen 10/IX/2014; BR-485, 12/XI/2014, 26/I/2015.

Gall (Fig. 180): on bud and leaf, globoid, red, hairy, and one-chambered. Galler: not determined. Paths: Travessia Ruy Braga, 08/VII/2014; BR-485, 26/I/2015.

No previous gall records on this plant species. Machaerium Pers. sp. $(\mathrm{n}=1)$

Gall (Fig. 181): on bud, conical, flattened, green, and glabrous. Galler: not determined. Path: Travessia Ruy Braga, 08/VII/2014.

Previous gall records on undetermined species of Machaerium: 1) in Atlantic forest - Rübsaamen 1908 ( $\mathrm{n}=1 /$ Tubarão/SC, n=1/Rio de Janeiro/RJ), Tavares 1916 ( $\mathrm{n}=1 /$ Friburgo/RJ), Tavares 1920 $(\mathrm{n}=2 /$ Friburgo/RJ), Fernandes et al. $2001(\mathrm{n}=1 /$ Vale do Rio Doce/MG), Fernandes and Negreiros 2006 (n=1/Aimorés/MG), Maia 2013b (n=1/ São Tomé das Letras/MG), Maia et al. 2014 (n=1/ Santa Teresa/ES), Maia and Carvalho-Fernandes 2016 (n=6/São Francisco de Itabapoana/RJ), 2) in Amazonian forest: - Almada and Fernandes 2011 ( $\mathrm{n}=2 /$ Oriximiná/PA).

Mimosa candollei R.Grether (native species, NE) $(\mathrm{n}=1)$

Gall (Fig. 182): on bud, globoid, brown, and glabrous. Galler: not determined. Path: Travessia Serra Negra, 14-15/X/2014.

No previous gall records on this plant species. Mimosa itatiaiensis Dusén (endemic in Brazil, Atlantic Florest, NE) ( $\mathrm{n}=1)$

Gall (Fig. 183): on apical and lateral bud, globoid, brown, pubescent, and multichambered (Fig.). Galler: not determined. Parasitoids: Hymenoptera (adult fragments). Path: Cinco Lagos, 11-12/V/2015.

No previous gall records on this plant species.
Mimosa melanocarpa Benth. (endemic in Brazil, Cerrado, NE) (first record in RJ and Atlantic forest) $(n=4)$

Gall (Fig. 184): on stem, fusiform, rusty, glabrous, and curved. Galler: not determined. Inquilines: Hemiptera. Paths: BR-485, 09/ XII/2014; Ecoarte-Lago Azul, 08/IV/2014.

Gall (Fig. 185): on stem, fusiform, green or brown, straight, with brown grooves and small spines. Galler: Cecidomyiidae (02 larvae). Paths: BR-485, 09/XII/2014; Travessia Ruy Braga, 08/ VII/2014; Mirante do Barbosa Rodrigues-Mirante do Último Adeus, 09/XII/2014.

Gall(Fig. 186): on leafand bud, globoid, yellow, with trichomes (red basally and green apically), and one-chambered. Galler: Cecidomyiidae (01 larva, 01 pupal exuviae). Parasitoids: Hymenoptera (01 adult). Paths: Centro de Visitantes, 07-08/VII/2014; Três Picos, 11/XI/2014; BR-485, 23/II/2015.

Gall (Fig. 187): on leaf, globoid, brown, glabrous, and one-chambered. Galler: not determined (empty galls). Path: BR-485, 23/ II/2015.

No previous gall records on this plant species. Mimosa L. sp. $(\mathrm{n}=1)$

Gall (Fig. 188): on bud, conical, widest apically, with string projections, greenish, glabrous, and one-chambered. Galler: Cecidomyiidae (02 larvae). Path: Viúva Hansen, 10/IX/2014.

Previous gall records on this plant genus: in caatinga - Maia et al. 2010 on Mimosa tenuiflora (Willd.) Poir (as Mimosa hostiles) $(\mathrm{n}=2 /$ Parnamirim/PE).

\section{LAMIACEAE $(\mathrm{N}=6)$}

Aegiphila Jacq. sp. (native genus) ( $\mathrm{n}=1)$

Gall (Fig. 189): on leaf, globoid, brown, glabrous, and one-chambered. Galler: Cecidomyiidae (02 larvae). Path: Viúva Hansen, 10/IX/2014.

No previous records on this plant genus. 
Hesperozygis myrtoides (A. St.-Hil. Ex Benth.) Epling (endemic in Brazil, Atlantic forest, NE) $(\mathrm{n}=1)$

Gall (Fig. 190): on bud, ovoid, brown, glabrous, and one-chambered. Galler: Lepidoptera. Path: Travessia Ruy Braga, 13/V/2015.

No previous gall records on this plant.

Rhabdocaulon coccineum (Benth.) Epling (endemic in Brazil, Atlantic forest, NE) $(n=1)$

Gall (Fig. 191): on bud, fusiform, green, glabrous, spongy internally, and multichambered. Galler: not determined (empty galls). Path: Travessia Ruy Braga, 13/V/2015.

No previous gall records on this plant.

Salvia oligantha Dúsen (endemic in Brazil, Atlantic forest, NE) $(n=1)$

Gall (Fig. 192): on leaf, globoid, yellow, pubescent, and one-chambered. Galler: not determined. Path: Travessia Ruy Braga, 17/ III/2015.

No previous gall records on this plant.

Salvia sellowiana Benth. (endemic in Brazil, Atlantic forest, NE) $(n=2)$

Gall (Fig. 193): on leaf vein, fusiform, orange, and one-chambered. Galler: not determined. Associated fauna: Hymenoptera (01 adult). Path: Casa 33, 16/VI/2015.

Gall (Fig. 194): on leaf, cylindrical, green, pubescent, and onechambered. Galler: not determined. Path: Casa 33, 16/VI/2015.

No previous gall records on this plant.

\section{LAURACEAE $(\mathrm{N}=5)$}

Nectandra membranacea (Sw.) (native species, $\mathrm{NE})(\mathrm{n}=1)$

Gall (no Fig.): on leaf, globoid, reddish, with trichomes, and one-chambered. Galler: not determined. Path: Barbosa Rodrigues, 09/XII/2014.

No previous gall records on this plant species. Nectandra Rol. ex Rottb. sp. (native genus) ( $\mathrm{n}=1$ )
Gall (Fig. 195): on leaf, globoid, yellow, and glabrous. Galler: not determined. Path: Travessia Serra Negra, 14-15/X/2014.

Previous gall records on undetermined species of this genus: 1) in Atlantic forest - Maia 2014 ( $n=1 /$ Itamonte/MG), Maia et al. 2014 ( $n=1 /$ Santa Teresa/ES), 2) no biome data - Mendonça et al. 2014 ( $\mathrm{n}=2 / \mathrm{RS})$.

Ocotea Aubl. sp. (native genus) $(\mathrm{n}=2)$

Gall (Fig. 196): on leaf, globoid, greenish, glabrous, and one-chambered. Galler: Hemiptera. Path: Casa de Pedra, 25/II/2014.

Gall (no fig.): on bud, globoid, reddish, glabrous, and multichambered. Galler: not determined. Path: Travessia Ruy Braga, 24/II/2015.

Previous gall records on undetermined species of this genus: 1) in Atlantic Forest - Maia 2013b ( $\mathrm{n}=1 /$ São Tomé das Letras/MG), Maia et al. 2014 $(n=1 /$ Santa Teresa/ES), 2) in Cerrado - Maia and Fernandes 2004 (n=1/Serra de São José/MG), 3) in Amazonian forest - Almada and Fernandes 2011 ( $\mathrm{n}=1 /$ Oriximiná/PA), Araújo et al. 2012 ( $\mathrm{n}=6 /$ Oriximiná/PA, 4) no biome data - Mendonça et al. 2014 ( $\mathrm{n}=2 / \mathrm{RS})$.

Phyllostemonodaphne geminiflora (Mez) Kosterm (native species, LC) $(\mathrm{n}=1)$

Gall (no fig.): on leaf, globoid, green, glabrous, and one-chambered. Galler: not determined. Parasitoids: Hymenoptera (01 adult, 03 pupae). Path: Travessia Ruy Braga, 24/II/2015.

No previous gall records on this plant species.

\section{LORANTHACEAE $(\mathrm{N}=09)$}

Phthirusa Mart. sp. 1 (native genus) ( $\mathrm{n}=4)$

Gall (Fig. 197): on aerial root, conical, brown, and glabrous. Galler: not determined. Path: Travessia Serra Negra, 14-15/X/2014.

Gall (Fig. 198): on leaf, conical, reddish, glabrous, and one-chambered. Galler: not determined. Parasitoids: Hymenoptera (02 pupae, 

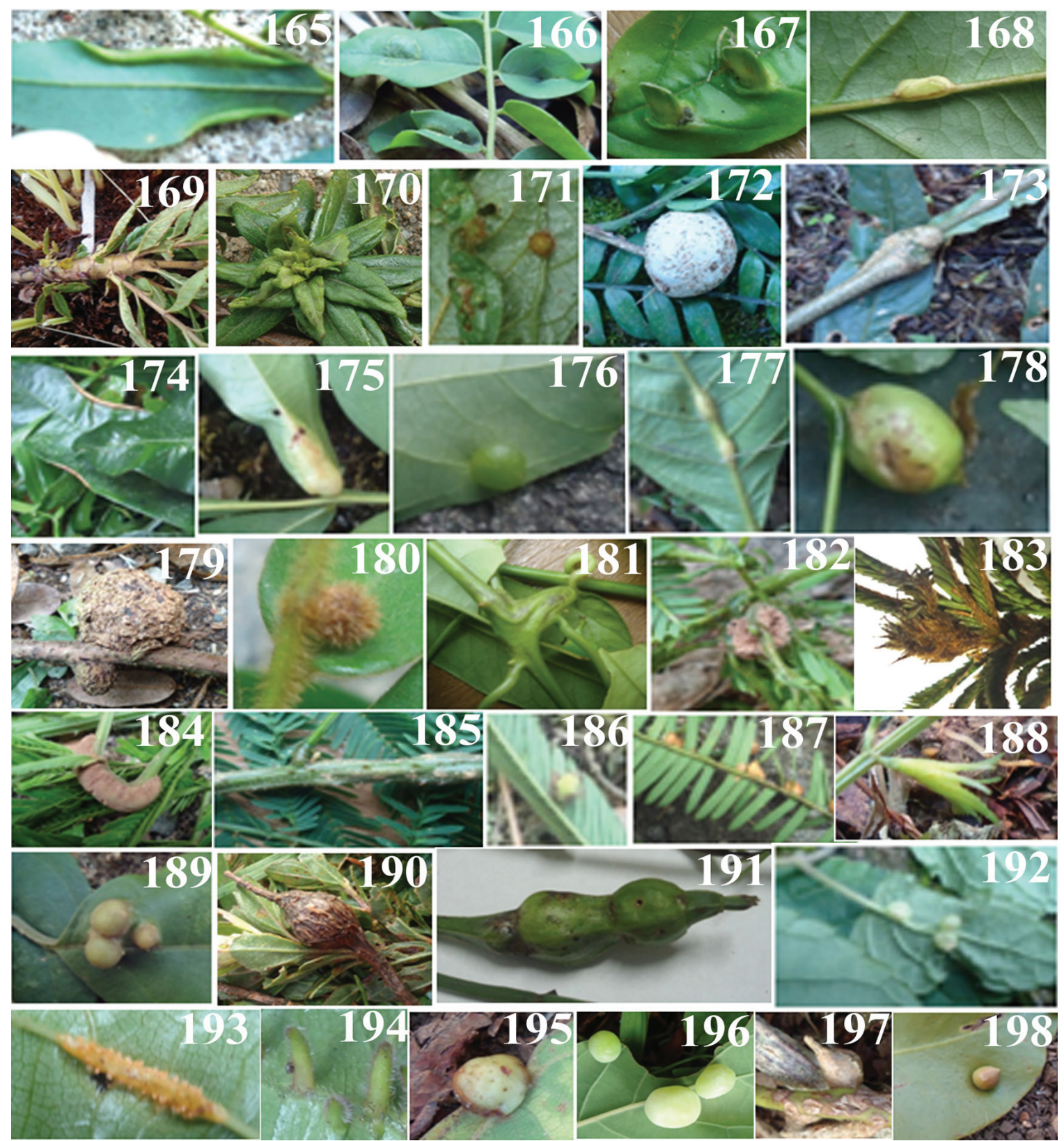

Figure 165-198 - Insect galls of the Parque Nacional do Itatiaia (Southeast Region, Brazil), 165-188, on Fabaceae, 165, Dalbergia foliosa, marginal leaf roll, 166, D. subcymosa, coalescent leaf gall, 167-168, Derris sp., 167, leaf fold, 168, fusiform leaf vein gall, 169-170, Grazielia gaudichaudeana, 160, fusiform stem gall, 170, rosette bud gall, 171, Inga barbata, globoid leaf gall, 172, I. gracilifolia, globoid bud gall, 173-174, I. grandiflora, 173, fusiform stem, leaf petiole and vein gall, 174, marginal leaf roll, 175176, I. marginata, 175, fusiform leaf midvein gall, 176, globoid leaf gall, 177, Inga cf. sessilis, fusiform leaf midvein gall, 178, Luetzelburgia auriculata, globoid bud gall, 179-180, Machaerium nyctitans, 179, globoid stem gall, 180, globoid bud and leaf gall, 181, Machaerium sp., conical bud gall, 182, Mimosa candollei, globoid bud gall, 183, M. itatiayensis, globoid bud gall, 184174, M. melanocarpa, 184, fusiform stem gall, 185, fusiform stem gall, 186, globoid bud and leaf gall, 187, globoid leaf gall, 188, Mimosa sp., conical bud gall, 189-194, on Lamiaceae, 189, Aegiphila sp., globoid leaf gall, 190, Hesperozygis myrtoides, ovoid bud gall, 191, Rhabdocaulon coccineum, fusiform bud gall, 192, Salvia oligantha, globoid leaf gall, 193-194, S. sellowiana, 193, fusiform leaf vein gall, 194, cylindrical leaf gall, 195-196, on Lauraceae, 195, Nectandra sp., globoid leaf gall, 196, Ocotea sp., globoid leaf gall, 197-198, on Loranthaceae, Phthirusa sp.1, 197, conical aerial root gall, 198, conical leaf gall. 
01 adult). Path: Travessia Serra Negra, 14-15/ $\mathrm{X} / 2014$.

Gall (Fig. 199): on bud and leaf, globoid, green, glabrous, and one-chambered. Galler: not determined. Parasitoids: Hymenoptera (02 pupae, 01 adult). Path: Travessia Serra Negra, 14-15/ $\mathrm{X} / 2014$.

Gall (Fig. 200): on leaf, globoid, brown, glabrous, and one-chambered. Galler: not determined. Parasitoids: Hymenoptera (01 pupa). Path: Água Branca-Ruy Braga, 18/VI/2014.

Phthirusa Mart.sp. 2 ( $\mathrm{n}=2)$

Gall (Fig. 201): on leaf, globoid, green, and glabrous. Galler: not determined. Path: Travessia Serra Negra, 14-15/X/2014.

Gall (Fig. 202): on stem, fusiform, brown, and glabrous. Galler: not determined. Path: Travessia Serra Negra, 14-15/X/2014.

Previous gall records on this genus: in Atlantic forest - Maia 2014 ( $\mathrm{n}=4 /$ Itamonte/MG).

Struthanthus concinnus (Mart.) Mart. (endemic in Brazil, Caatinga, Cerrado, Amazonian and Atlantic forest, NE) and Struthanthus pentamerus Rizzini (endemic in Brazil, Atlantic forest, occurrence only in RJ) (NE) (n=3)*

Gall (Fig. 203): on leaf and bud, globoid, yellow, glabrous, and one-chambered. Galler: Cecidomyiidae (01 larva). Path: Travessia Ruy Braga, 15/IX/2015.

Gall (Fig. 204): on bud, ovoid, with a leaflike apical projection, green, glabrous, and onechambered. Galler: Cecidomyiidae (01 larva). Inquilines: Lepidoptera. Path: Travessia Ruy Braga, 15/IX/2015.

Gall (Fig. 205): on leaf, fusiform, yellowish, with trichomes, and one-chambered. Galler: not determined. Path: Travessia Ruy Braga, 15/ IX/2015.

* As both species were collected as they were a single taxon, we could not determine which one hosted which gall morphotype.
Previous gallrecordson S. concinnus: Rodrigues et al. 2014 recorded other two morphoypes, one on root induced by Cecidomyiidae and the other on leaf and stem induced by Asphondylia sp. (Cecidomyiidae) in Marambaia, RJ.

No previous gall records on $S$. pentamerus.

\section{MALPIGHIACEAE $(\mathrm{N}=3)$}

Byrsonima Rich. ex Kunth. sp. (native genus) $(\mathrm{n}=1)$

Gall (Fig. 206): on leaf, conical, rusty, pubescence, and one-chambered. Galler: not determined (empty galls). Path: BR-485, 04/ VIII/2014.

Previous gall records on undetermined species of this genus: 1) in Atlantic forest - Fernandes et al. 1997 ( $n=3 /$ Vale do Jequitinhonha/MG), Santos et al. $2011 \mathrm{~b}(\mathrm{n}=1 / \mathrm{six}$ municipalities/PE), Maia et al. 2014 ( $\mathrm{n}=3 /$ Santa Teresa/ES), 2) in Cerrado - Maia and Fernandes 2004 (n=1/Serra de São José/MG), Araújo et al. 2011 ( $n=1 /$ Pirenópolis, GO), Carneiro et al. 2009b ( $n=3 /$ Serra do Espinhaço/MG), Coelho et al. 2013b ( $\mathrm{n}=1 /$ Serra do Cabral/MG), Araújo et al. $2015(n=4 / G O), 3)$ in Amazonian forest Almada and Fernandes 2011 ( $\mathrm{n}=1 /$ Oriximiná/PA), 4) in Caatinga - Santos et al. 2011a ( $\mathrm{n}=1 / \mathrm{PE})$.

Heteropterys Kunth. sp. (native genus) $(\mathrm{n}=1)$

Gall (Fig. 207): on aerial root, fusiform, brown, and glabrous. Galler: not determined. Path: Água Branca-Ruy Braga, 18/VI/2014.

Previous gall records on this genus: in Atlantic forest - on H. nitida: Maia 2001 (n=1/Maricá/RJ).

Niedenzuella acutifolia (Cav.) W.R.Anderson (native species, NE) $(\mathrm{n}=1)$

Gall (Fig. 208): on leaf, globoid, yellow, glabrous, and one-chambered. Galler: not determined (empty galls). Paths: Barbosa Rodrigues-Mirante do Último Adeus, 09/XII/2014; BR-485, 28/I/2015, 23/II/2015.

No previous gall records on this genus. 


\section{MELASTOMATACEAE $(\mathrm{N}=66)$}

Bellucia Raf. sp. (native genus) (first record in Southeast Region and Atlantic forest) $(n=3)$

Gall (Fig. 209): on bud, rough, globoid, green, glabrous, and one-chambered. Galler: Lopesia sp. (Cecidomyiidae) (01 larva). Path: Travessia Ruy Braga, 04/VIII/2014.

Gall (Fig. 210): on leaf midvein, fusiform, green, glabrous, and one-chambered. Galler: Lepidoptera (01 pupa). Parasitoids: Hymenoptera (01 larva). Paths: Água Branca-Ruy Braga, 18/ VI/2014; Travessia Ruy Braga, 04/VIII/2014, Três Picos, 09/IX/2014.

Gall (Fig. 211): on leaf, fold, green, glabrous, and one-chambered. Galler: not determined (empty galls). Path: Travessia Ruy Braga, 04/VIII/2014.

Previous gall records on this genus: in Amazonian forest - on B. grossularioides (L.) Triana: Almada and Fernandes $2011 \quad(n=1 /$ Oriximiná/PA), Maia 2011 ( $\mathrm{n}=1 /$ Oriximiná/PA), on B. imperialis Saldanha and Cogn. - Almada and Fernandes 2011 (n=1/Oriximiná/PA/Amazonian forest).

Clidemia D. Don. sp.1 (native genus) $(\mathrm{n}=2)$

Gall (Fig. 212): on bud, leaf blade and petiole, globoid, hairy, green, and one-chambered. Galler: Lopesia sp. (Cecidomyiidae) (04 pupae, 01 pupal exuvia, 09 larvae). Parasitoids: Hymenoptera (01 larva, 04 adults). Inquilines: Thysanoptera (02 adults). Paths: Cachoeira Itaporani, 17/III/2014, 12/V/2014; Cachoeira Véu da Noiva, 17/III/2014; Três Picos, 13/V/2014; 11/XI/2014; Casa 16, 07/ VII/2014; Travessia Ruy Braga, 08/VII/2014, 08/ XII/2014，05/VIII/2014; 16/III/2015; DonatiSimon 2, 08/IX/2014; Viúva Hansen, 10/IX/2014; Travessia Serra Negra, 14-15/X/2014; Barbosa Rodrigues-Mirante do Último Adeus, 09/XII/2014; Barbosa Rodrigues (obelisk), 10/XII/2014; BR485, 26/I/2015, 23/II/2015; Cachoeira do Pitu, 25/ II/2015.
Gall (Fig. 213): on bud and leaf, fusiform, green, with many long brown trichomes, and onechambered. Galler: Lepidoptera (03 catterpilars). Path: Travessia Ruy Braga, 08/VII/2014.

Clidemia D. Don. sp. $2(\mathrm{n}=1)$

Gall (Fig. 214): on bud, globoid, green, with red pubescence, and one-chambered. Galler: Cecidomyiidae (01 larva). Path: Hotel Donati, 04/ VIII/2014.

Clidemia D. Don. sp.3 ( $\mathrm{n}=1)$

Gall (Fig. 215): on leaf blade and petiole, globoid, hairy, rusty, and one-chambered. Galler: Cecidomyiidae (01 larva). Path: BR-485, 12/ $\mathrm{XI} / 2014$.

Previous gall records on undetermined species of Clidemia: 1) in Atlantic forest - Santos et al. 2011b ( $\mathrm{n}=1 /$ six municipalities/PE), Maia 2013b ( $\mathrm{n}=3 /$ São Tomé das Letras/MG), Maia et al. 2014 ( $\mathrm{n}=1 /$ Santa Teresa/ES), Maia 2014 ( $\mathrm{n}=4 /$ Itamonte/ $\mathrm{MG})$.

Leandra hirta Raddi (endemic in Brazil, Atlantic forest, NE) $(\mathrm{n}=2)$

Gall (Fig. 216): on stem, fusiform, brown, with trichomes, and one-chambered. Galler: Cecidomyiidae (1 adult). Path: BR-485, 26/I/2015.

Gall (Fig. 217): on leaf, globoid, green, with trichomes, and one-chambered. Galler: not determined (empty galls). Path: BR-485, 26/I/2015.

No previous gall records on this plant species. Leandra sericea DC. (endemic in Brazil, Atlantic forest, NE) $(\mathrm{n}=1)$

Gall (Fig. 218): on leaf, globoid, with trichomes. Galler: Cecidomyiidae. Path: BR-485, 26/I/2015.

No previous gall records on this plant species. Leandra sulfurea (Naudin) Cogn. (endemic in Brazil, Cerrado and Atlantic forest, NE) $(n=1)$

Gall (no fig.): on leaf midvein, fusiform, green, glabrous, and one-chambered. Galler: Lepidoptera (01 pupa). Path: Pedra do Camelo, 14/IX/2015.

No previous gall records on this plant species. 
Maieta guianensis Aubl (native species) (first record in Southeast Region and Atlantic forest, NE) $(\mathrm{n}=1)$

Gall (Fig. 219): on leaf vein, fusiform, reddish, glabrous, and one-chambered. Galler: Lepidoptera (02 catterpilars). Paths: Lago Azul, 18/III/2014; Cachoeira Poranga, 07/IV/2014.

No previous gall records on this plant species. Miconia ceramicarpa (DC.) Cogn. (native species, $\mathrm{NE}$ ) (first record in Southeast Region and Atlantic forest) $(n=2)$

Gall (Fig. 220): on stem, fusiform, brown, glabrous, and multichambered. Galler: Contarinia sp. (Cecidomyiidae) (01 larva). Paths: EcoarteLago Azul, 08/IV/2014; Cachoeira Itaporani, 12/V/2014.

Gall (Fig. 221): on leaf, globoid, green or red, with wittish or greenish trichomes, and onechambered. Galler: Cecidomyiidae (01 pupa). Path: Três Picos, 08/XI/2014.

No previous gall records on this plant species. Miconia chrysophylla (Rich.) Urb. (native species, NE) (first record in Southeast Region and Atlantic forest) $(n=1)$

Gall (Fig. 222): on leaf, globoid, yellow, with brown trichomes, and one-chambered. Galler: Cecidomyiidae (01 larva). Paths: Água BrancaRuy Braga, 18/VI/2014; Travessia Ruy Braga, 18/ VI/2014.

No previous gall records on this plant species. Miconia cuspidata Naudin (native species, NE) (first record in RJ) ( $\mathrm{n}=1$ )

Gall (Fig. 223): on bud, conical, flattened, green, glabrous, and one-chambered. Galler: Lopesiini (Cecidomyiidae) (02 larvae). Parasitoids: Hymenoptera (02 adults). Paths: Travessia Ruy Braga, 08/VII/2014; Viúva Hansen, 10/IX/2014; Centro de Visitantes, 09/XII/2014; BR-485, 26/I/2015.

No previous gall records on this plant species.
Miconia myriantha Benth. (native species, NE) (first record in Southeast Region and Atlantic forest $)(n=3)$

Gall (Fig. 224): on stem and leaf petiole, globoid, brown, glabrous, and one-chambered. Galler: Lepidoptera (01 catterpilar). Path: Travessia Ruy Braga, 08/VII/2014.

Gall (Fig. 225): on bud, globoid, green, glabrous, with rough surface, and one-chambered. Galler: Lepidoptera (01 catterpilar). Paths: BR485, 04/VIII/2014, 12/XI/2014; Viúva Hansen, 10/ $\mathrm{IX} / 2014$.

Gall (Fig. 226): on leaf, globoid, green, glabrous, and one-chambered. Galler: not determined (empty galls). Paths: Cachoeira Véu da Noiva, 17/III/2014; Viúva Hansen, 10/IX/2014.

No previous gall records on this plant species. Miconia paucidens DC. (endemic in Brazil, Cerrado, LC) (first record in RJ and Atlantic forest) $(\mathrm{n}=2)$

Gall (Fig. 227): on bud, globoid, green, with green or red micropubescence. Galler: Lepidoptera (2 catterpilars). Path: Ecoarte-Lago Azul, 08/ IV/2014.

Gall (Fig. 228): on leaf, globoid, green, with red trichomes, and one-chambered. Galler: not determined. Path: Travessia Ruy Braga, 08/ $\mathrm{VII} / 2014$.

No previous gall records on this plant species. Miconia pusilliflora (DC.) Naudin (native species, $\mathrm{NE})(\mathrm{n}=1)$

Gall (Fig. 229): on stem, globoid, green, glabrous, and one-chambered. Galler: Lepidoptera (02 catterpilars). Path: Travessia Ruy Braga, 08/ VII/2014.

Previous gall records in this plant species: in Atlantic forest - Maia et al. 2008 ( $n=1 /$ Bertioga), Toma and Mendonça 2013 (n=1/São Francisco de Paula/RS).

Miconia urophylla DC. (endemic in Brazil, Atlantic forest, NE) $(\mathrm{n}=2)$ 

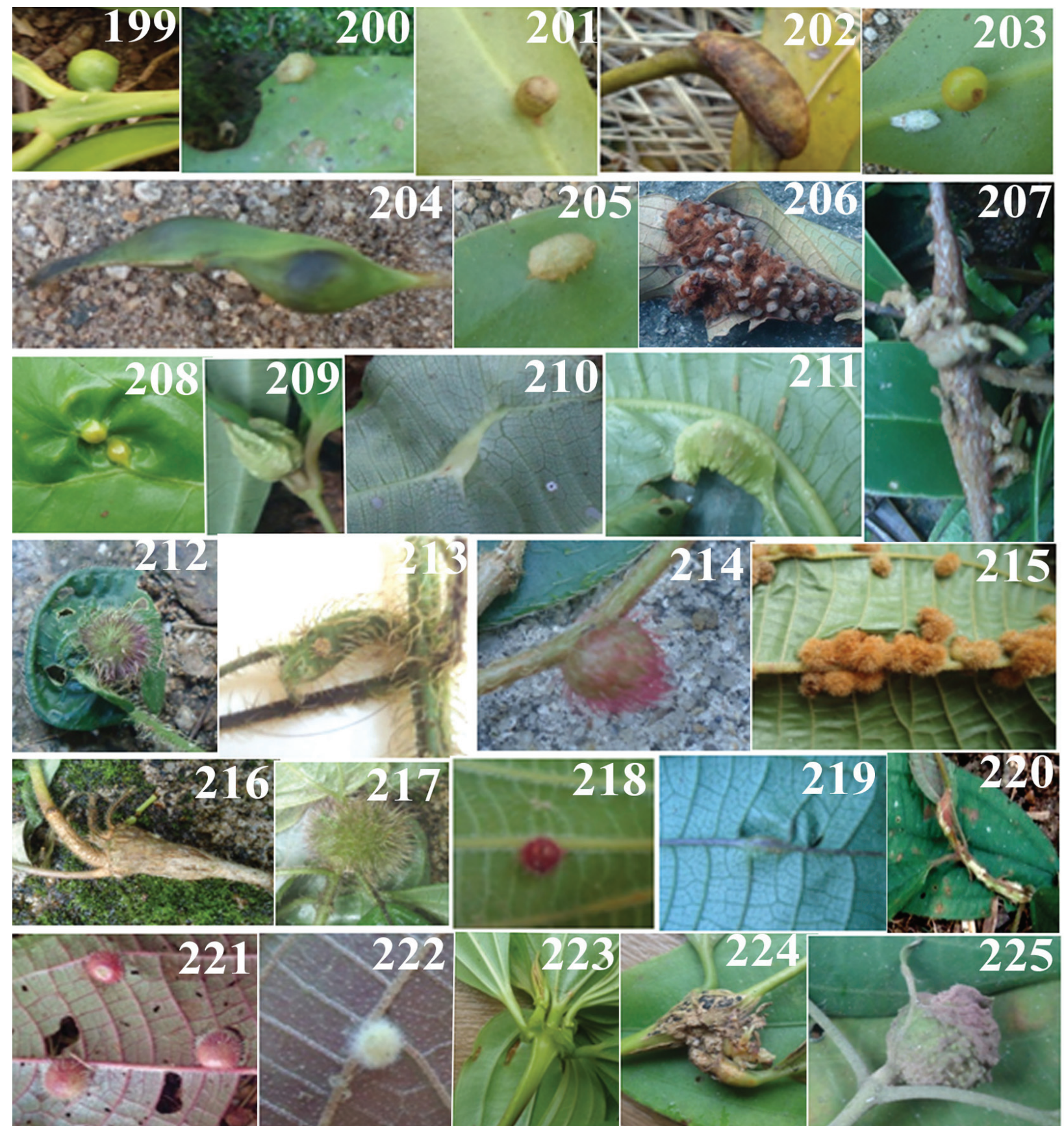

Figure 199-225 - Insect galls of the Parque Nacional do Itatiaia (Southeast Region, Brazil), 199-205, on Loranthaceae, 199-200, Phthirusa sp.1, 199, globoid bud and leaf gall, 200, globoid leaf gall, 201-202, Phthirusa sp.2, 201, globoid leaf gall, 202, fusiform stem gall, 203-205, Struthanthus concinnus and S. pentamerus, 203, globoid bud and leaf gall, 204, ovoid bud gall, 205, fusiform leaf gall, 206-208, on Malpighiaceae, 206, Byrsonima sp., conical leaf gall, 207, Heteropterys sp., fusiform aerial root gall, 208, Niedenzuella acutifolia, globoid leaf gall, 209-225, on Melastomataceae, 209-211, Bellucia sp., 209, globoid bud gall, 210, fusiform leaf midvein gall, 211, leaf fold, 212-213, Clidemia sp.1, 212, globoid bud gall, 213, fusiform bud gall, 214, Clidemia sp.2, globoid bud gall, 215, Clidemia sp.3, globoid leaf blade and petiole gall, 216-217, Leandra hirta, 216, fusiform stem gall, 217 , globoid leaf gall, 218, L. sericea, globoid leaf gall, 219, Maieta guianensis, fusiform leaf vein gall, 220-221, Miconia ceramicarpa, 220, fusiform stem gall, 221, globoid leaf gall, 222, M. chrysophylla, globoid leaf gall, 223, M. cuspidata, conical bud gall, 224225, M. myriantha, 224, globoid stem and leaf petiole gall, 225, globoid bud gall. 
Gall (Fig. 230): on bud, ovoid, reddish, glabrous, and multichambered. Galler: not determined (empty gall). Path: Casa 25, 08/ $\mathrm{XII} / 2015$.

Gall (Fig. 231): on stem, fusiform, brown, and glabrous. Galler: not determined (empty gall). Path: Casa 25, 08/XII/2015.

No previous gall records on this plant species. Miconia Ruiz and Pav. sp.1 ( $\mathrm{n}=2)$

Gall (Fig. 232): on leaf midvein, fusiform, green, glabrous, and one-chambered. Galler: Lepidoptera (04 catterpilars). Parasitoids: Hymenoptera (02 larvae). Paths: Três Picos, 13/V/2014, 11/XI/2014; Água Branca-Ruy Braga, 18/VI/2014; Travessia Ruy Braga, 24/II/2015. Similar gall recorded on Miconia sp. by Maia 2014 in the PNI (Brejo da Lapa).

Gall (Fig. 233): on leaf petiole, conical, green, and glabrous. Galler: not determined (empty gall). Path: Cachoeira Itaporani, 17 /III/2014.

Miconia Ruiz and Pav. sp.2 ( $\mathrm{n}=2)$

Gall(Fig. 234): on leaf, conical, green, glabrous, and one-chambered. Galler: Cecidomyiidae (02 gregarious larvae). Path: Cachoeira Itaporani, 12/V/2014.

Gall (Fig. 235): on leaf, globoid, rust, brown with brown pubescence, and one-chambered. Galler: not determined. Parasitoids: Hymenoptera (02 larvae, 02 pupae). Paths: Cachoeira Véu da Noiva, 17/III/2014; Cachoeira Poranga, 07/ IV/2014.

Miconia Ruiz and Pav. sp.3 ( $\mathrm{n}=2$ )

Gall (Fig. 236): on bud, globoid, green, with red pubescence, and one-chambered. Galler: Lepidoptera (03 catterpilars). Inquilines: Muscomorpha (01 larva). Paths: Cachoeira Poranga, 07/IV/2014; Casa 16, 07/VII/2014; Travessia Ruy Braga, 08/VII/2014; Viúva Hansen, 10/IX/2014.

Gall (Fig. 237): on stem, fusiform, woody, brown, glabrous, and one-chambered. Path: Viúva Hansen, 10/IX/2014.

Miconia Ruiz and Pav. sp.4 (n=4)
Gall (Fig. 238): on stem, fusiform, whittish, and one-chambered. Galler: Cecidomyiidae (01 young larva). Paths: Travessia Serra Negra, 14-15/ X/2014; Travessia Ruy Braga, 17/III/2015.

Gall (Fig. 239): on leaf vein, fusiform, green, glabrous, and one-chambered. Galler: not determined. Path: Travessia Ruy Braga, 14/ IV/2015, 15/IX/2015.

Gall (Fig. 240): on leaf, ovoid, green, with thin projections, and one-chambered. Galler: not determined Parasitoids: Hymenoptera (01 pupa). Paths: Água Branca-Ruy Braga, 18/VI/2014, 14/ IV/2015; Travessia Serra Negra, 14-15/X/2014; Três Picos, 11/XI/2014; Travessia Ruy Braga, 24/ II/2015, 17/III/2015, 16/IV/2015.

Gall (Fig. 241): on bud, ovoid, green, glabrous, and multichambered. Galler: not determined (empty galls). Paths: Travessia Serra Negra, 1415/X/2014; Travessia Ruy Braga, 17/III/2015.

Miconia Ruiz and Pav. sp.5 ( $\mathrm{n}=1)$

Gall (Fig. 242): on leaf, globoid, thorny, green, and one-chambered. Galler: Cecidomyiidae (02 young larvae). Paths: Água Branca-Ruy Braga, 18/VI/2014, 14/IV/2015; Travessia Serra Negra, $14-15 / \mathrm{X} / 2014$.

Miconia Ruiz and Pav. sp.6 ( $\mathrm{n}=1)$

Gall (Fig. 243): on stem, fusiform, green, pubescent, and one-chambered. Galler: not determined. Path: Travessia Ruy Braga, 08/ VII/2014.

Miconia Ruiz and Pav. sp.7 ( $\mathrm{n}=1)$

Gall (Fig. 244): on leaf vein, fusiform, reddish, glabrous, and one-chambered. Galler: not determined (empty galls). Path: Travessia Serra Negra, 14-15/X/2014.

Miconia Ruiz and Pav. sp. $8(\mathrm{n}=1)$

Gall (Fig. 245): on leaf, globoid or cylindrical, yellow, glabrous, and one-chambered. Galler: Cecidomyiidae (01 larva, 01 pupa). Parasitoids: Hymenoptera (02 larvae, 04 adults). Paths: Travessia Ruy Braga, 24/II/2015; Cachoeira do Pitu, 25/II/2015. 
Previous gall records on undetermined Miconia: 1) in Atlantic forest - Santos et al. 2012 ( $\mathrm{n}=1 / \mathrm{PE}$ ), Maia 2013b ( $\mathrm{n}=6 / \mathrm{São}$ Tomé das Letras/ MG), Maia et al. 2014 ( $n=5 /$ Santa Teresa/ES), Maia 2014 (n=8/Itamonte/MG), 2) in Cerrado Maia and Fernandes 2004 ( $\mathrm{n}=1 /$ Serra de São José/MG), Carneiro et al. 2009b ( $n=4 /$ Serra do Espinhaço/MG), Malves and Frieiro-Costa 2012 ( $\mathrm{n}=5 /$ Ingaí/MG), 3) in Amazonian forest - Almada and Fernandes 2011 (n=2/Oriximiná/PA/), Araújo et al. 2012 ( $\mathrm{n}=3 /$ Oriximiná/PA).

Tibouchina estrellensis (Raddi) Cogn. (endemic in Brazil, Atlantic forest, NE) $(n=2)$

Gall (Fig. 246): on bud rosette, green, glabrous, and one-chambered. Galler: Lepidoptera (01 catterpilar, 01 pupa). Paths: BR-485, 28/I/2015, Casa 25, 08/XII/2015.

Gall (Fig. 247): on leaf midvein, fusiform, brown, pubescent, and one-chambered. Galler: not determined (empty galls). Path: Casa 25, 08/ XII/2015.

No previous gall records on this plant species.

Tibouchina fothergillae (Schrank and Mart. ex DC.) Cogn. (endemic in Brazil, Atlantic forest, $\mathrm{NE})(\mathrm{n}=1)$

Gall (Fig. 248): on leaf midvein, fusiform, green, glabrous, and one-chambered. Galler: Lepidoptera (fragments). Path: Água Branca -Ruy Braga, 18/VI/2014.

No previous gall records on this plant species. Tibouchina hospita Cogn. (endemic in Brazil, Atlantic forest, NE) $(n=3)$

Gall (Fig. 249): on bud, globoid, brown, glabrous, and one-chambered. Galler: Cecidomyiidae (01 larva). Path: Travessia Ruy Braga, 13/V/2015.

Gall (Fig. 250): on stem, fusiform, brown, glabrous, and one-chambered. Galler: Cecidomyiidae (01 larva). Parasitoids: Hymenoptera. Path: Asa de Hermes, 16/IX/2015.

Gall (Fig. 251): on bud, fusiform, green or reddish, pubescent, and one-chambered. Galler:
Lepidoptera (01 catterpilar). Path: Travessia Ruy Braga, 13/V/2015.

Previous record of this plant species: 1) in Atlantic forest (altitude fields) - Coelho et al. 2013a (n=1/Itatiaia).

Tibouchina cf. martiusiana (DC.) Cogn. (endemic in Brazil, Cerrado and Atlantic forest, NE) $(n=2)$

Gall (Fig. 252): on leaf vein, ovoid, yellowish, and one-chambered. Galler: Lepidoptera (01 pupa). Paths: Prateleiras, 09/IV/2014; Pedra do Camelo, 14/IX/2015.

Gall (Fig. 253): on bud, fusiform, red, micropubescent, and one-chambered. Galler: not determined. Parasitoids: Hymenoptera (04 adults). Path: Travessia Serra Negra, 14-15/X/2014.

Previous gall records on this plant species: in Atlantic Forest - Fernandes et al. 2001 ( $n=2 /$ Vale do Rio Doce/MG).

Tibouchina semidecandra (Schrank and Mart. ex DC.) Cogn. (endemic in Brazil, Cerrado and Atlantic forest, NE) $(n=6)$

Gall (Fig. 254): on bud, rosette, green or yellow with red pubescenc. Galler: Cecidomyiidae (01 larva). Inquilines: Coleoptera (01 larva). Path: Água Branca-Ruy Braga, 18/VI/2014.

Gall (Fig. 255): on bud, conical, red, with trichomes, and one-chambered. Galler: Cecidomyiidae (01 larva). Path: Água Branca-Ruy Braga, 18/VI/2014.

Gall (Fig. 256): on bud, globoid, reddish, with trichomes, and one-chambered. Galler: Lepidoptera (01 catterpilar). Path: Água BrancaRuy Braga, 18/VI/2014.

Gall (Fig. 257): on leaf, ovoid, green, with reddish trichomes, and one-chambered. Galler: not determined. Path: Água Branca-Ruy Braga, 18/ $\mathrm{VI} / 2014$.

Gall (Fig. 258): on stem, fusiform, brown, glabrous, and multichambered. Galler: not determined (empty galls). Path: Travessia Serra Negra, 14-15/X/2014. 

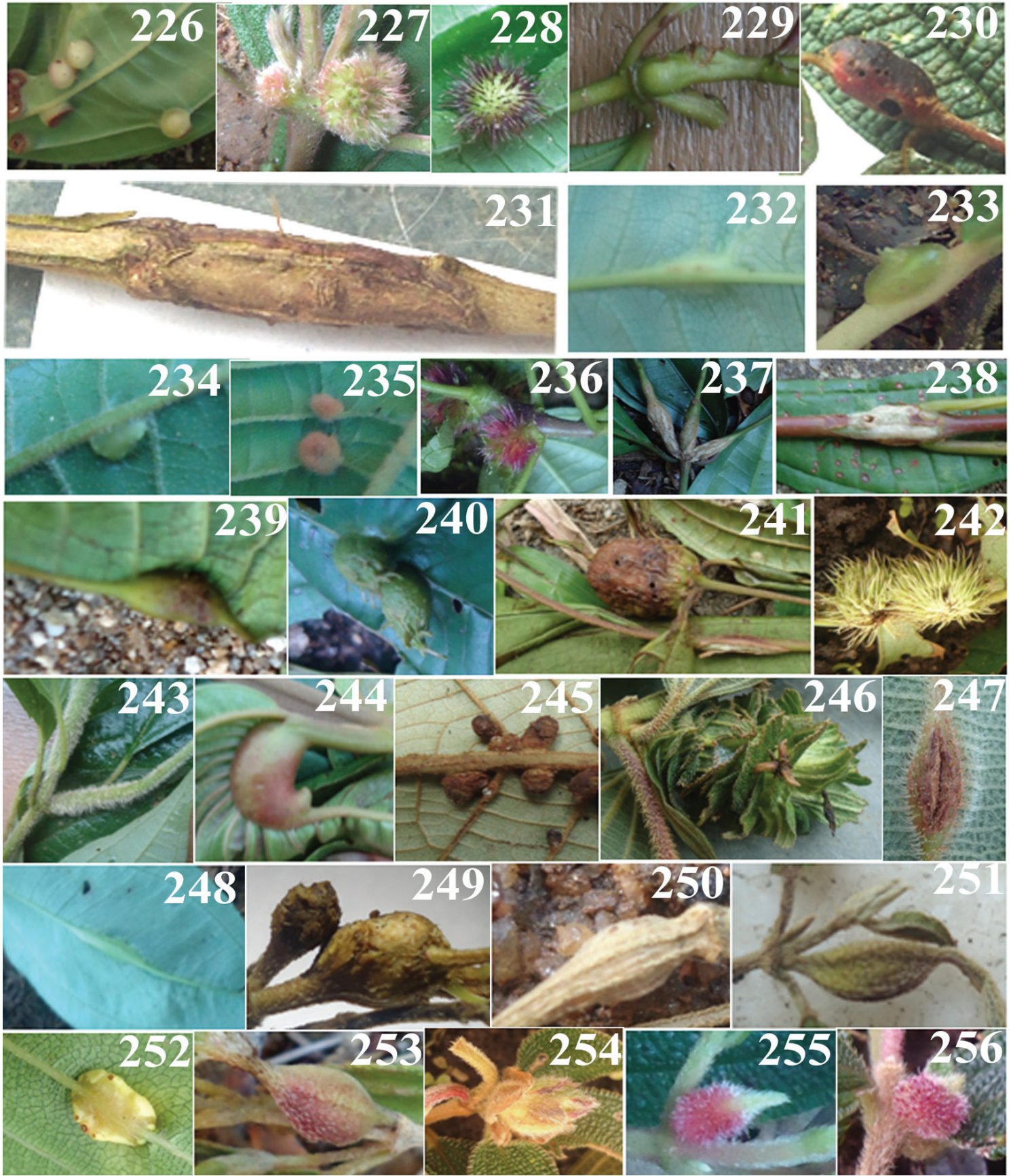

Figure 226-256 - Insect galls of the Parque Nacional do Itatiaia (Southeast Region, Brazil), on Melastomataceae, 226, Miconia cuspidata, globoid leaf gall, 227-228, M. paucidens, 227, globoid bud gall, 228, globoid leaf gall, 229, M. passiliflora, globoid stem gall, 230-231, M. cerophylla, 230, ovoid bud gall, 231, fusiform stem gall, 232-233, Miconia sp.1, 232, fusiform leaf midvein gall, 233, conical leaf petiole gall, 234-235, Miconia sp. 2, 234, conical leaf gall, 235, globoid leaf gall, 236-237, Miconia sp. 3, 236, globoid bud gall, 237, fusiform stem gall, 238-241, Miconia sp. 4, 238, fusiform stem gall, 239, fusiform leaf vein gall, 240, ovoid leaf gall, 241, ovoid bud gall, 242, Miconia sp. 5, globoid leaf gall, 243, Miconia sp. 6, fusiform stem gall, 244, Miconia sp. 7, fusiform leaf vein gall, 245, Miconia sp. 8, globoid or cylindrical leaf gall, 246-247, Tibouchina estrellensis, 246, rosette bud gall, 247, fusiform leaf midvein gall, 248, T. fothergillae, fusiform leaf midvein gall, 249-251, T. hospita, 249, globoid bud gall, 250, fusiform stem gall, 251, fusiform bud gall, 252-253, Tibouchina cf. martiusiana, 252, ovoid leaf vein gall, 253, fusiform bud gall, 254-256, T. semidecandra, 254, rosette bud gall, 255, conical bud gall, 256, globoid bud gall. 
Gall (Fig. 259): on apical bud, globoid, tapered apically, brown, glabrous, and solid. Galler: not determined. Path: Travessia Serra Negra, 14-15/ $\mathrm{X} / 2014$.

No previous gall records.

Tibouchina Aubl. sp. 1 ( $\mathrm{n}=2)$

Gall (Fig. 260): on lateral bud, cylindrical, green, with trichomes. Galler: Lepidoptera (01 caterpillar). Path: Travessia Ruy Braga, 18/ VI/2014, 14/IV/2015.

Gall (no fig.): on leaf, globoid, yellow with white trichomes, and one-chambered. Galler: Cecidomyiidae (01 larva). Path: Água Branca-Ruy Braga, 18/VI/2014.

Tibouchina Aubl. sp.2 (n=1)

Gall (Fig. 261): on stem, fusiform, brown, and multichambered. Galler: Cecidomyiidae (02 adults). Path: Barbosa Rodrigues-Mirante do Último Adeus, 09/XII/2014.

Tibouchina Aubl. sp.3 ( $\mathrm{n}=3)$

Gall (Fig. 262): on bud, globoid, brown, with trichomes, and one-chambered. Galler: Lepidoptera. Path: Viúva Hansen, 10/IX/2014.

Gall (Fig. 263): on leaf, globoid, green or reddish, with white trichomes, and one-chambered. Galler: not determined. Path: Viúva Hansen, 10/ IX/2014.

Gall (Fig. 264): on stem, fusiform, brown, glabrous, and one-chambered. Galler: not determined. Associated fauna: Hymenopteara (parasitoids - fragments). Paths: Viúva Hansen, 10/ IX/2014; BR-485, 26/I/2015.

Tibouchina Aubl. sp.4 (n=3)

Gall (Fig. 265): on vein, fusiform, sinuous, green, glabrous, and one-chambered. Galler: Lepidoptera (01 pupa). Path: Travessia Serra Negra, 14-15/X/2014.

Gall (Fig. 266): on leaf petiole, fusiform, irregular in width, green, glabrous, and onechambered. Galler: Lepidoptera (silk and pupal exuvia fragments). Path: Travessia Serra Negra, $14-15 / \mathrm{X} / 2014$.
Gall (Fig. 267): on leaf petiole, fusiform, regular in width, green, glabrous, and onechambered. Galler: Lepidoptera (silk and pupal exuvia fragments). Path: Travessia Serra Negra, $14-15 / \mathrm{X} / 2014$.

Tibouchina Aubl. sp.5 ( $\mathrm{n}=1)$

Gall (Fig. 268): on bud, globoid, red, with yellow pubescence. Galler: Lopesiini (Cecidomyiidae) (01 larva). Path: Travessia Serra Negra, 14-15/X/2014.

Tibouchina Aubl. sp.6 ( $\mathrm{n}=1)$

Gall (Fig. 269): on stem, ovoid, grooved, green or red, glabrous, and one-chambered. Galler: not determined (empty galls). Path: Travessia Serra Negra, 14-15/X/2014.

Previous gall records on undetermined Tibouchina Aubl.: 1) in Atlantic forest - Fernandes et al. $2001(\mathrm{n}=5 /$ Vale do Rio Doce/MG), Maia et al. 2014 (n=1/Santa Teresa/ES), 2) no biome data Mendonça et al. 2014 ( $n=2 / R S)$.

Not determined $1(n=2)$

Gall (Fig. 270): on leaf, globoid, greenish, with long trichomes, and one-chambered. Galler: not determined. Path: BR-485, 04/VIII/2014.

Gall (Fig. 271): on leaf petiole and midvein, fusiform, green, and pubescent. Galler: not determined. Path: Água Branca-Ruy Braga, 18/ $\mathrm{VI} / 2014$.

\section{Not determined $2(\mathrm{n}=1)$}

Gall(Fig. 272): on bud, rosette, green, glabrous, and one-chambered. Galler: not determined. Path: Água Branca-Ruy Braga, 18/VI/2014.

\section{MELIACEAE $(\mathrm{N}=4)$}

Guarea F. Allam. ex L. sp. (native genus) $(\mathrm{n}=1)$

Gall (Fig. 273): on leaf, marginal roll, green, glabrous, and one-chambered. Galler: Hemiptera. Paths: Lago Azul, 18/III/ 2014; Barbosa RodriguesMirante do Último Adeus, 09/XII/2014; BR-485, 28/I/2015; Travessia Ruy Braga, 16/III/2015. 
Previous gall records on this plant genus: 1) in Atlantic forest: on Guarea guidonia (L.) Sleumer - Fernandes et al. 2001 ( $n=3 /$ Vale do Rio Doce/ MG), Rodrigues et al. 2014 ( $n=3 /$ Mangaratiba/ RJ), Maia et al. 2014 ( $\mathrm{n}=1 /$ Santa Teresa/ES), on G. macrophylla Vahl. subsp tuberculata (Vell.) T. D. Penn - Maia et al. 2008 ( $n=3 /$ Bertioga/SP), on Guarea sp.- Santos et al. 2012 (n=1/PE), Fernandes et al. 2001 ( $n=2 / /$ Vale do Rio Doce/MG), Santos et al. $2012(\mathrm{n}=2 / \mathrm{PE})$, Maia et al. $2014(\mathrm{n}=1 /$ Santa Teresa/ES), 2) in Pampa: on Guarea sp., possibly $G$. trichilioides L. - Tavares 1909 (n=1/São Leopoldo/ RS).

Trichilia P. Browne sp. (native genus) $(\mathrm{n}=3)$

Gall (Fig. 274): on stem, fusiform, green, glabrous, and one-chambered. Galler: Lepidoptera. Path: BR-485, 12/XI/2014.

Gall (Fig. 275): on leaf, fold, green, glabrous, and one-chambered. Galler: Hemiptera (14 specimens). Parasitoids: Hymenoptera (01 adult). Paths: Ecoarte-Lago Azul, 08/IV/2014; BR-485, 26/I/2015.

Gall (Fig. 276): on stem, globoid, brown, and glabrous. Galler: not determined (empty galls). Path: BR-485, 12/XI/2014.

Previous gall records on this plant genus: 1) in Atlantic forest: on T. quadrijuga (Miq.) Kunth - Santos et al. 2012 (n=1/PE), on T. elegans A. Juss. ( $\mathrm{n}=1)$, T. rubra C. DC $(\mathrm{n}=1)$ and Trichilia spp. $(\mathrm{n}=7)$ - Maia and Carvalho-Fernandes 2016 (São Francisco de Itabapoana/RJ), 2) in Amazonian forest: on T. appendiculata (Triana and Planch.) C. DC. $(\mathrm{n}=1)$ and on Trichilia sp. $(\mathrm{n}=3)$ - Araújo et al. 2012 (Oriximiná/PA), 3) in Caatinga-Cerrado: on T. emarginata (Turcz.) C. DC. - Costa et al. 2015 $(\mathrm{n}=2 /$ Caetité/BA $), 4)$ no biome data - Mendonça et al. 2014 ( $n=3 / R S)$.

\section{MENISPERMACEAE $(\mathrm{N}=1)$}

Disciphania Eichler sp. (native genus) $(\mathrm{n}=1)$
Gall (Fig. 277): on stem, globoid, brown, glabrous, and one-chambered. Galler: Cecidomyiidae (01 larva). Path: BR-485, 23/ II/2015.

No previous gall records in this plant genus.

Previous gall records in this plant family: 1) in Pantanal: on Hyperbaena hasslerii Diels $(\mathrm{n}=1)$ and Odontocarya fanoides (DC.) Myers ( $\mathrm{n}=1$ ) - Julião et al. 2001 (MS), 2) in Amazonian forest: on Abuta grandifolia (Mart.) Sandwith - Maia 2011 ( $\mathrm{n}=1 /$ Oriximiná/PA).

\section{MONIMIACEAE $(\mathrm{N}=1)$}

Mollinedia Ruiz and Pav. sp. (native genus) ( $\mathrm{n}=1$ )

Gall (Fig. 278): on leaf, marginal roll, green, glabrous, and one-chambered. Galler: not determined. Parasitoids: Hymenoptera (02 adults). Sucessors: Formicidae (01 adult). Path: Travessia Ruy Braga, 17/III/2015.

Previous gall records on undetermined species of this plant genus: 1) in Atlantic forest - Santos et al. 2011b ( $\mathrm{n}=1 /$ six municipalities/PE), 2) no biome data - Mendonça et al. 2014 ( $n=1 / R S)$.

\section{MORACEAE $(\mathrm{N}=1)$}

Ficus L. sp. (native genus) ( $\mathrm{n}=1)$

Gall (Fig. 279): on leaf, discoid, yellow peripherically and reddish in the middle, glabrous, and one-chambered. Galler: Cecidomyiidae(exuvia fragments). Parasitoids: Hymenoptera (fragments). Path: Travessia Ruy Braga, 15/IV/2015.

Previous gall records on undetermined species of this plant genus: 1) in Atlantic forest - Tavares 1917 (n=2/Itaparica and São Antonio da Barra/ BA and Friburgo/RJ), Rodrigues et al. 2014 ( $n=1 /$ Mangaratiba/RJ), Maia and Carvalho-Fernandes 2016 (n=2/São Francisco de Itabapoana/RJ), 2) in Pantanal - Julião et al. 2001 ( $\mathrm{n}=2 / \mathrm{MS})$.

\section{MYRTACEAE $(\mathrm{N}=26)$}

Calyptranthes Sw.sp. (native genus) $(\mathrm{n}=1)$ 
Gall (Fig. 280): on leaf, globoid, near midvein, green, glabrous, and one-chambered. Galler: not determined. Parasitoids: Hymenoptera (01 pupa). Paths: Cachoeira Véu da Noiva, 17/III/2014; BR485, 23/II/2015.

Previous gall records on undetermined species of this plant genus: 1) in Atlantic forest - Monteiro et al. 2004 ( $n=1 / J u r u b a t i b a / R J)$, Maia et al. 2008 ( $n=3 /$ Bertioga/SP), Santos et al. 2012 ( $n=2 / P E), 2)$ no biome data - Mendonça et al. 2014 ( $\mathrm{n}=1 / \mathrm{RS})$.

Eugenia bunchosiifolia Nied. (endemic in Brazil, Atlantic forest, VU) $(\mathrm{n}=2)$

Gall (Fig. 281): on leaf, rounded and brown at basis, with a central green cylinder, glabrous, and one-chambered. When the gall dries, the cylinder falls and the basis remains. Galler: Cecidomyiidae (01 young larva). Paths: Viúva Hansen, 10/ IX/2014; Mirante do Barbosa Rodrigues-Mirante do Último Adeus, 09/XII/2014; Cachoeira do Pitu, 25/II/2015.

Gall (Fig. 282): on bud, globoid, brown, glabrous, and four-chambered. Galler: not determined (empty gall). Path: Cachoeira Itaporani, $12 / \mathrm{V} / 2014$.

Previous gall records on this plant species: in Atlantic forest - Maia and Carvalho-Fernandes 2016 (n=2/São Francisco de Itabapoana/RJ).

Eugenia schottiana O.Berg. (endemic in Brazil, Caatinga and Atlantic forest, NE) $(n=1)$

Gall(Fig.283): on leaf, discoid, green, glabrous, and one-chambered. Galler: Cecidomyiidae (01 larva). Path: Travessia Ruy Braga, 24/II/2015.

No previous gall records on this plant species. Eugenia uniflora L. (native species, NE) ( $\mathrm{n}=1)$

Gall(Fig.284): on leaf, discoid, green, glabrous, and one-chambered. Galler: Neolasioptera eugeniae Maia, 1993. Path: BR 485, 23/II/2015.

Previous gall records of the same morphotype: in Atlantic forest - Maia 2001 (Maricá/RJ), Oliveira and Maia 2005 (Rio de Janeiro/RJ), Maia and Oliveira 2010 (Angra dos Reis/RJ), Maia 2013a (Paraty and Cabo Frio/RJ), Maia and Souza 2013
(Arraial do Cabo/RJ), Maia 2014 (Itamonte/MG), Maia and Silva 2016 (Rio de Janeiro/RJ).

Eugenia L. sp. 1 (native genus) $(\mathrm{n}=3$ )

Gall (Fig. 285): on bud, globoid, with reduced apical leaf, green or brown, and glabrous. Galler: Cecidomyiidae (03 pupal exuvia). Path: Travessia Serra Negra, 14-15/X/2014.

Gall (Fig. 286): on leaf, marginal roll, green, glabrous, and one-chambered. Galler: not determined (empty galls). Path: Travessia Serra Negra, 14-15/X/2014.

Gall (Fig. 287): on stem, fusiform, brown, glabrous, and one-chambered. Galler: not determined. Path: Travessia Ruy Braga, 14/ $\mathrm{IV} / 2015$.

Eugenia L. sp. 2 ( $\mathrm{n}=1)$

Gall (no fig.): on leaf, globoid, green, glabrous, and one-chambered. Galler: not determined. Parasitoids: Hymenoptera (17 adults). Path: Cachoeira do Pitu, 25/II/2015.

Previous gall records on undetermined species of Eugenia L.: 1) in Atlantic forest - Tavares 1920 ( $n=1 /$ Madre de Deus/Bahia), Oliveira and Maia 2005 ( $\mathrm{n}=3 /$ Rio de Janeiro/RJ), Maia and Oliveira 2010 ( $\mathrm{n}=3 /$ Angra dos Reis/RJ), Santos et al. 2011b $(\mathrm{n}=1 /$ six municipalities/PE), Santos et al. 2012 $(\mathrm{n}=1 / \mathrm{PE})$, Maia 2013b ( $\mathrm{n}=1 /$ São Tomé das Letras/ MG), Maia et al. 2014 ( $n=2 /$ Santa Teresa/ES), Maia and Carvalho-Fernandes 2016 ( $\mathrm{n}=5 /$ São Francisco de Itabapoana), 2) in Cerrado - Gonçalves-Alvim and Fernandes 2001 ( $n=3 /$ Três Marias/MG), Malves and Frieiro-Costa 2012 ( $\mathrm{n}=5 /$ Ingaí/MG), Luz et al, 2012 ( $\mathrm{n}=3 / \mathrm{Januária/MG),} \mathrm{Araújo} \mathrm{et} \mathrm{al.}$ 2014 ( $\mathrm{n}=1 /$ Mineiros/GO).

Myrcia fenzliana O.Berg (native species, NE) (first record in $\mathrm{RJ})(\mathrm{n}=1)$

Gall (no fig.): on leaf, globoid, green, glabrous, and one-chambered. Galler: not determined. Path: Travessia Ruy Braga, 08/VII/2014.

No previous gall records on this plant species. 

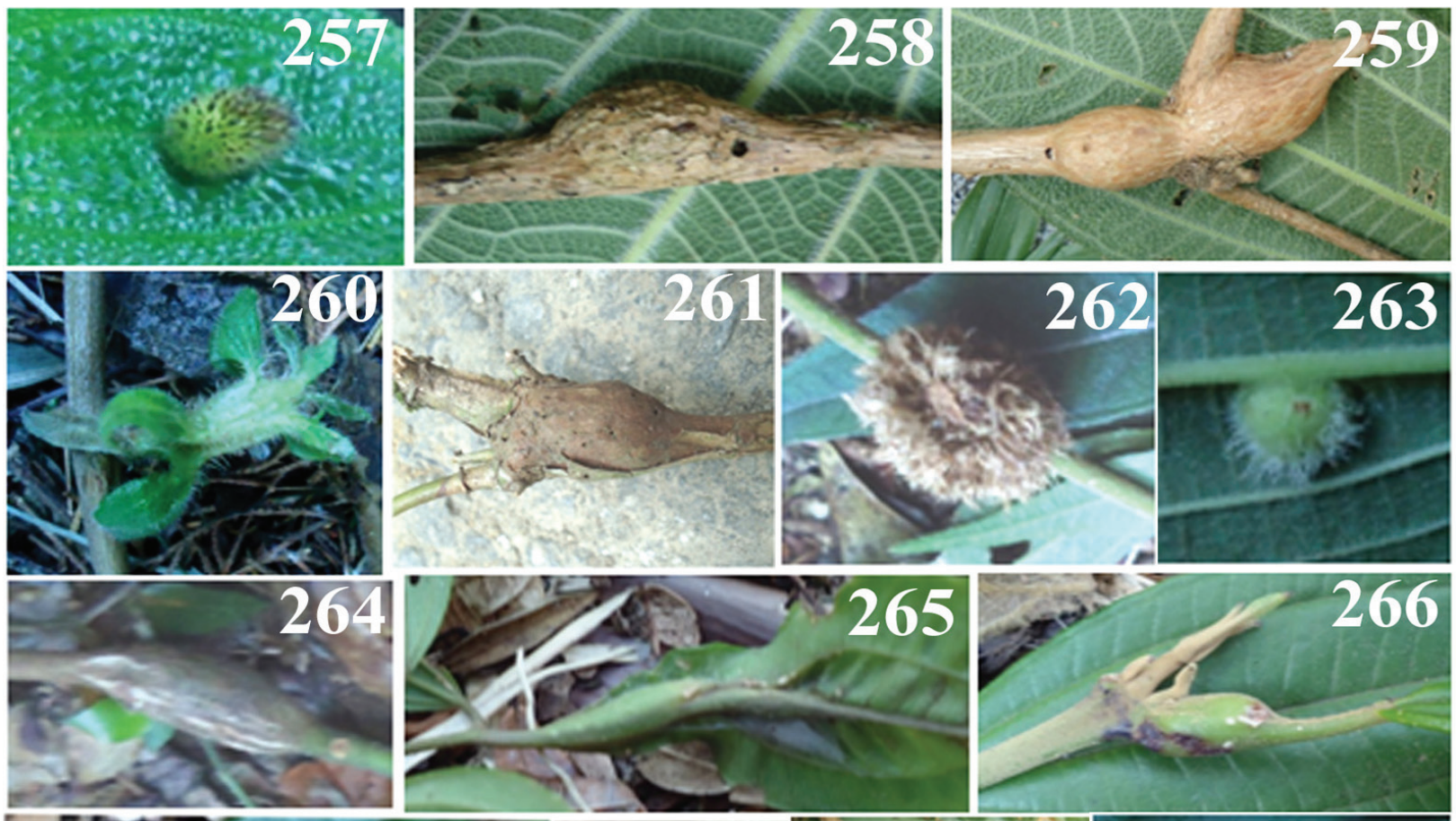

263
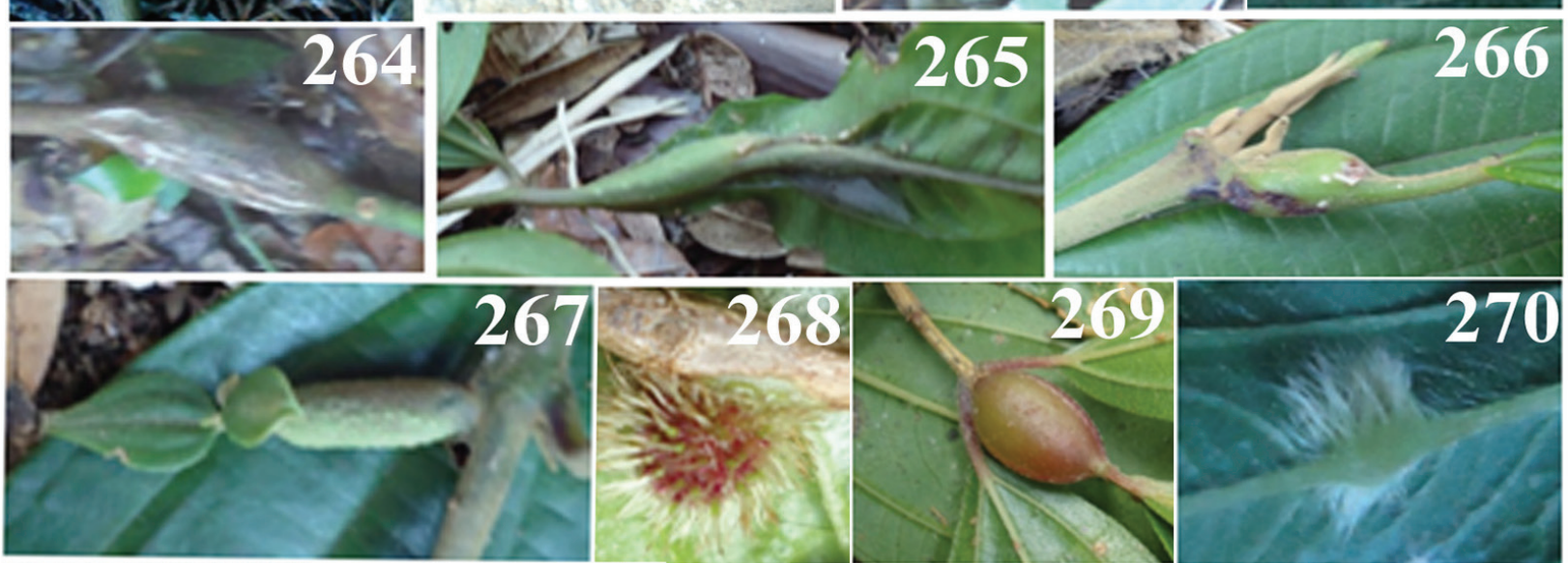

270
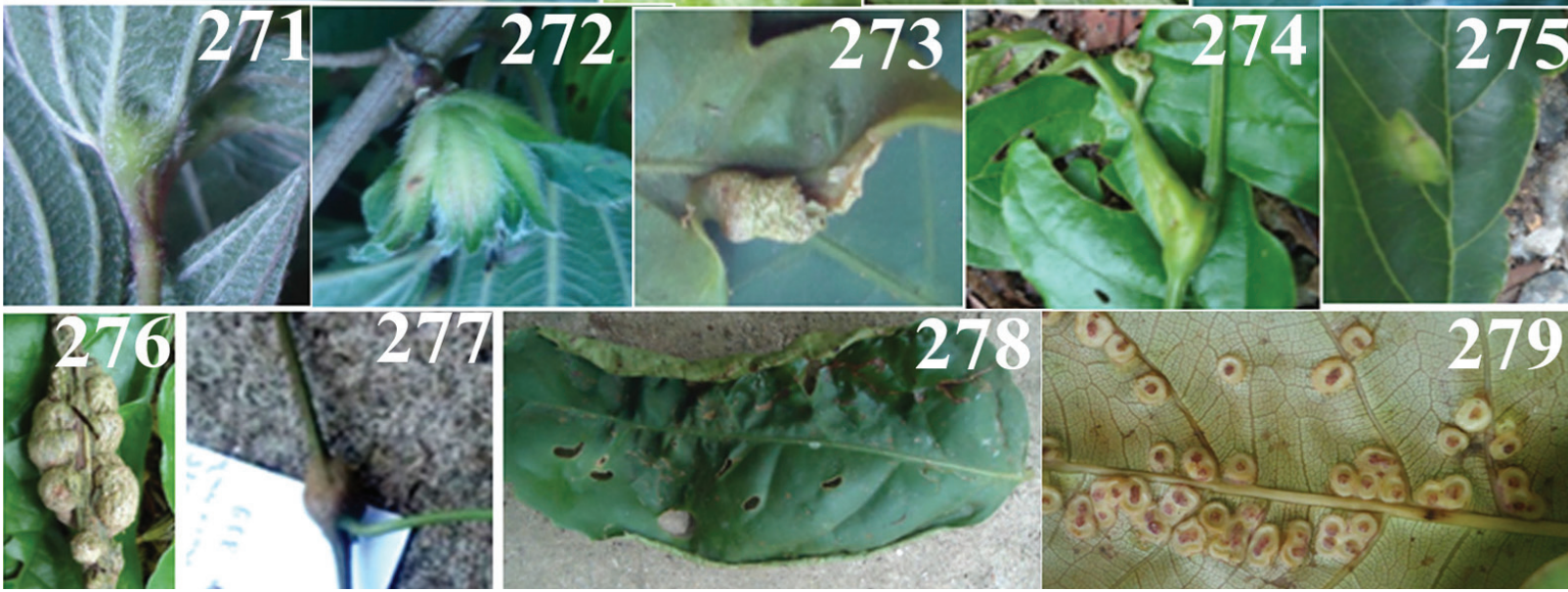

Figure 257-279 - Insect galls of the Parque Nacional do Itatiaia (Southeast Region, Brazil), 257-272, on Melastomataceae, 257-259, Tibouchina semidecandra, 257, ovoid leaf gall, 258, fusiform stem gall, 259, globoid bud gall, 260, Tibouchina sp.1, cylindrical bud gall, 261, Tibouchina sp. 2, fusiform stem gall, 262-264, Tibouchina sp. 3, 262, globoid bud gall, 263, globoid leaf gall, 264, fusiform stem gall, 265-267, Tibouchina sp. 4, 265, fusiform leaf vein gall, 266, fusiform leaf petiole gall, 267, fusiform leaf petiole gall, 268, Tibouchina sp. 5, globoid bud gall, 269, Tibouchina sp. 6, ovoid stem gall, 270-271, Melastomataceae sp.1, 270, globoid leaf gall, 271, fusiform leaf petiole and midvein gall, 272, Melastomataceae sp. 2, rosette bud gall, 273-276, on Meliaceae, 273, Guarea sp., marginal leaf roll, 274-276, Trichilia sp., 274, fusiform stem gall, 275, leaf fold, 276, globoid stem gall, 277, on Menispermaceae, Disciphania sp., globoid stem gall, 278, on Monimiaceae, Mollinedia sp., marginal leaf roll, 279, on Moraceae, Ficus sp., discoid leaf gall. 
Myrcia splendens (Sw.) DC. (endemic in Brazil, Caatinga, Cerrado, Pantanal. Amazonian and Atlantic forest, NE) $(n=4)$

Gall (Fig. 288): on leaf, globoid, green, with trichomes, and one or bi-chambered. Galler: Cecidomyiidae (02 pupal exuvia, 04 larvae). Paths: Cachoeira Poranga, 07/IV/2014; Ecoarte-Lago Azul, 08/IV/2014; Donati-Simon 2, 08/IX/2014; Barbosa Rodrigues-Mirante do Último Adeus, 09/ XII/2014.

Gall (Fig. 289): on apical bud, globoid, green, glabrous, and three-chambered (01 larva by chamber). Galler: Cecidomyiidae (05 larvae). Paths: Três Picos, 01/IV/2014, 09/IX/2014, 11/ XI/2014; Travessia Ruy Braga, 08/XII/2014.

Gall (Fig. 290): on bud and leaf, globoid, glabrous, and two-chambered. Galler: Cecidomyiidae (02 larvae). Parasitoids: Hymenoptera (04 larvae, 01 pupa, 01 adult). Path: Travessia Ruy Braga, 08/XII/2014, 16/III/2015, 17/III/2015, 14/IV/2015.

Gall (Fig. 291): on leaf, roll, green, glabrous, and one-chambered. Galler: not determined. Path: Centro de Visitantes, 07-08/VII/2014.

Previous gall records on this plant species: 1) in Atlantic forest - Maia 2013b (n=2/São Tomé das Letras/MG), 2) in Cerrado - Malves and FrieiroCosta 2012 ( $\mathrm{n}=2 /$ Ingaí/MG), 3) in CaatingaCerrado - Costa et al. 2015 ( $\mathrm{n}=1 /$ Caetité/BA).

Myrcia cf. splendens (Sw.) DC. $(\mathrm{n}=2)$

Gall (Fig. 292): on bud, globoid, green or brown, glabrous, with apical thin projections, and one to three-chambered. Galler: Cecidomyiidae (02 larvae). Parasitoids: Hymenoptera (07 pupae, 01 adult). Paths: Água Branca-Ruy Braga, 18/ VI/2014; Três Picos, 11/XI/2014; Travessia Ruy Braga, 18/VI/2014, 24/II/2015.

Gall (Fig. 293): on leaf, globoid, green, glabrous, and one to three-chambered. Galler: Cecidomyiidae (03 larvae). Paths: Travessia Ruy Braga, 24/II/2015; Cachoeira do Pitu, 25/II/2015.
Myrcia sylvatica (G.Mey.) DC. (native species, $\mathrm{NE})(\mathrm{n}=8)$

Gall (Fig. 294): on leaf, marginal roll, red, glabrous, and one-chambered. Galler: Thysanoptera (22 specimens - adults and nymphs). Inquilines: Lepidoptera (02 catterpilars). Paths: Água BrancaRuy Braga, 18/VI/2014; Travessia Serra Negra, 14-15/X/2014; Três Picos, 11/XI/2014; Barbosa Rodrigues-Mirante do Último Adeus, 09/XII/2014; BR-485, 23/II/2015; Travessia Ruy Braga, 24/ II/2015.

Gall (Fig. 295): on stem, fusiform, green, red or brown, glabrous, and two-chambered. Galler: not determined (empty galls). Paths: Água BrancaRuy Braga, 18/VI/2014; Travessia Ruy Braga, 08/ VII/2014; Três Picos, 11/XI/2014; Cachoeira do Pitu, 25/II/2015.

Gall (Fig. 296): on bud and leaf, globoid, with a central depression, green, glabrous, and onechambered. Galler: not determined. Paths: Água Branca-Ruy Braga, 18/VI/2014; Travessia Ruy Braga, 14/IV/2015.

Gall (Fig. 297): on bud, globoid, reddish, and glabrous. Galler: Thysanoptera. Paths: Água Branca-Ruy Braga, 18/VI/2014; Três Picos, 11/ $\mathrm{XI} / 2014$.

Gall (Fig. 298): on bud and leaf basis, globoid, succulent, green, glabrous, and two-chambered. Galler: Cecidomyiidae (06 larvae, 02 pupal exuviae, 02 adults). Parasitoids: Hymenoptera (01 pupa, 06 adults). Paths: Água Branca-Ruy Braga, 18/VI/2014; Travessia Ruy Braga, 18/VI/2014; Três Picos, 09/IX/2014,11/XI/2014; Travessia Serra Negra, 14-15/X/2014.

Gall (Fig. 299): on bud, globoid, green, glabrous, and three-chambered (01 larva by chamber). Galler: Dasineura sp. (Cecidomyiidae) (01 male, 01 female, 02 pupal exuviae, 01 larva). Parasitoids: Hymenoptera (06 pupae). Paths: Água Branca-Ruy Braga, 18/VI/2014; Travessia Serra Negra, 14-15/X/2014; Travessia Ruy Braga, 08/ $\mathrm{XII} / 2014$. 
Gall (Fig. 300): on leaf midvein and petiole, globoid, woody, brown, glabrous, two-chambered. Galler: Dasineura sp. (Cecidomyiidae) (02 pupal exuviae, 02 females). Parasitoids: Hymenoptera (01 pupa). Path: Travessia Serra Negra, 14-15/X/2014.

Gall (Fig. 301): on leaf midvein, globoid, green or red, glabrous, and one-chambered to threechambered. Galler: Cecidomyiidae (19 larvae). Parasitoids: Hymenoptera. Paths: Travessia Ruy Braga, 18/VI/2014, 08/VII/2014, 10-12/IX/2014, BR-485, 12/XI/2014; Três Picos, 11/XI/2014; Barbosa Rodrigues (obelisk), 10/XII/2014.

Previous gall records on this plant species: in Atlantic forest - Santos et al. 2012 ( $n=2 / P E)$, Maia 2013b (n=7/São Tomé das Letras/MG).

Myrcia DC. sp.1 (native genus) $(\mathrm{n}=1)$

Gall (Fig. 302): on leaf, globoid, red with brown pubescence adaxially, and green abaxially. Galler: not determined (empty galls). Paths: Cachoeira Poranga, 07/IV/2014; Donati-Simon 2, 08/IX/2014.

Myrcia DC. sp.2(n=1)

Gall (Fig. 303): on stem, fusiform, brown, glabrous, and one-chambered. Galler: not determined. Path: Travessia Ruy Braga, 08/ VII/2014.

Previous gall records on undetermined species of Myrcia DC: 1) in Atlantic forest - Fernandes et al. 2001 ( $\mathrm{n}=4 /$ Vale do Rio Doce/MG), Santos et al. $2011 \mathrm{~b}$ ( $\mathrm{n}=4 /$ six municipalities/PE), Santos et al. 2012 (n=1/PE), Maia 2013b (n=3/São Tomé das Letras/MG), Maia et al. 2014 ( $\mathrm{n}=13 /$ Santa Teresa/ ES), Maia 2014 ( $\mathrm{n}=2 /$ Itamonte), 2) in Cerrado Santos et al. 2010 ( $\mathrm{n}=1 /$ Goiânia/GO), GonçalvesAlvim and Fernandes 2011 ( $\mathrm{n}=4 /$ Três Marias/MG), 3) in Caatinga -Santos et al. 2011a ( $\mathrm{n}=1 / \mathrm{PE})$.

NYCTAGINACEAE $(\mathrm{N}=9)$

Bougainvillea spectabilis Willd. (endemic in Brazil, Amazonian and Atlantic forest, NE) $(n=1)$
Gall (Fig. 304): on stem, globoid, brown, and glabrous. Galler: not determined. Muscomorpha (01 larva) and Lepidoptera (01 catterpilar). Paths: Água Branca-Ruy Braga, 18/VI/2014; Travessia Ruy Braga, 16/III/2015.

No previous gall records on this plant.

Guapira Aubl. sp.1 (native genus) $(\mathrm{n}=3$ )

Gall (Fig. 305): on bud and leaf, globoid, green, glabrous, and one-chambered. Galler: Bruggmannia sp. (Cecidomyiidae) (01 larva). Path: Ecoarte-Lago Azul, 08/IV/2014.

Gall (Fig. 306): on leaf, globoid, glabrous, yellow, and one-chambered. Galler: Cecidomyiidae (01 larva). Path: Três Picos, 13/V/2014.

Gall(Fig. 307): on leaf, discoid, green, glabrous, and one-chambered. Galler: not determined (empty gall). Path: Cachoeira Itaporani, 12/V/2014.

Previous gall records on this genus: 1) in Atlantic forest: on Guapira opposita (Vell.) Reitz - Maia 2001 (n=5/Maricá and Arraial do Cabo/RJ), Bregonci et al. 2010 ( $n=1 /$ Guarapari/ES), Santos et al. $2012(n=9 / P E)$, Maia and Souza $2013(n=4 /$ Arraial do Cabo/RJ), Maia et al. 2014 ( $n=8 /$ Santa Teresa/ES), Maia and Carvalho-Fernandes 2016 (n=3/São Francisco de Itabapoana/RJ), Maia and Silva 2016 (n=2/Rio de Janeiro/RJ), on Guapira pernambucensis (Casar) Lundell. - Oliveira and Maia 2005 ( $\mathrm{n}=1 /$ Rio de Janeiro/RJ), Bregonci et al. 2010 ( $\mathrm{n}=3 /$ Guarapari/ES), Maia and Souza 2013 (n=1/Arraial do Cabo/RJ), on Guapita nitida (Mart. ex J.A.Schmidt) Lundell - Maia et al. 2008/ Bertioga/SP), on Guapira sp. - Bregonci et al. 2010 ( $\mathrm{n}=1 /$ Guarapari/ES), Rodrigues et al. 2014 $(\mathrm{n}=8 /$ Mangaratiba/RJ), Santos et al. 2011b ( $\mathrm{n}=14$ / six municipalities/PE), Maia 2013b (n=1/São Tomé das Letras/MG), Maia and Carvalho-Fernandes 2016 (n=1/ São Francisco de Itabapoana/RJ), 2) in Cerrado: on Guapira sp. - Araújo et al. 2014 (n=3/ Mineiros/GO), Maia and Fernandes 2004 (n=2/ Serra de São José/MG). 

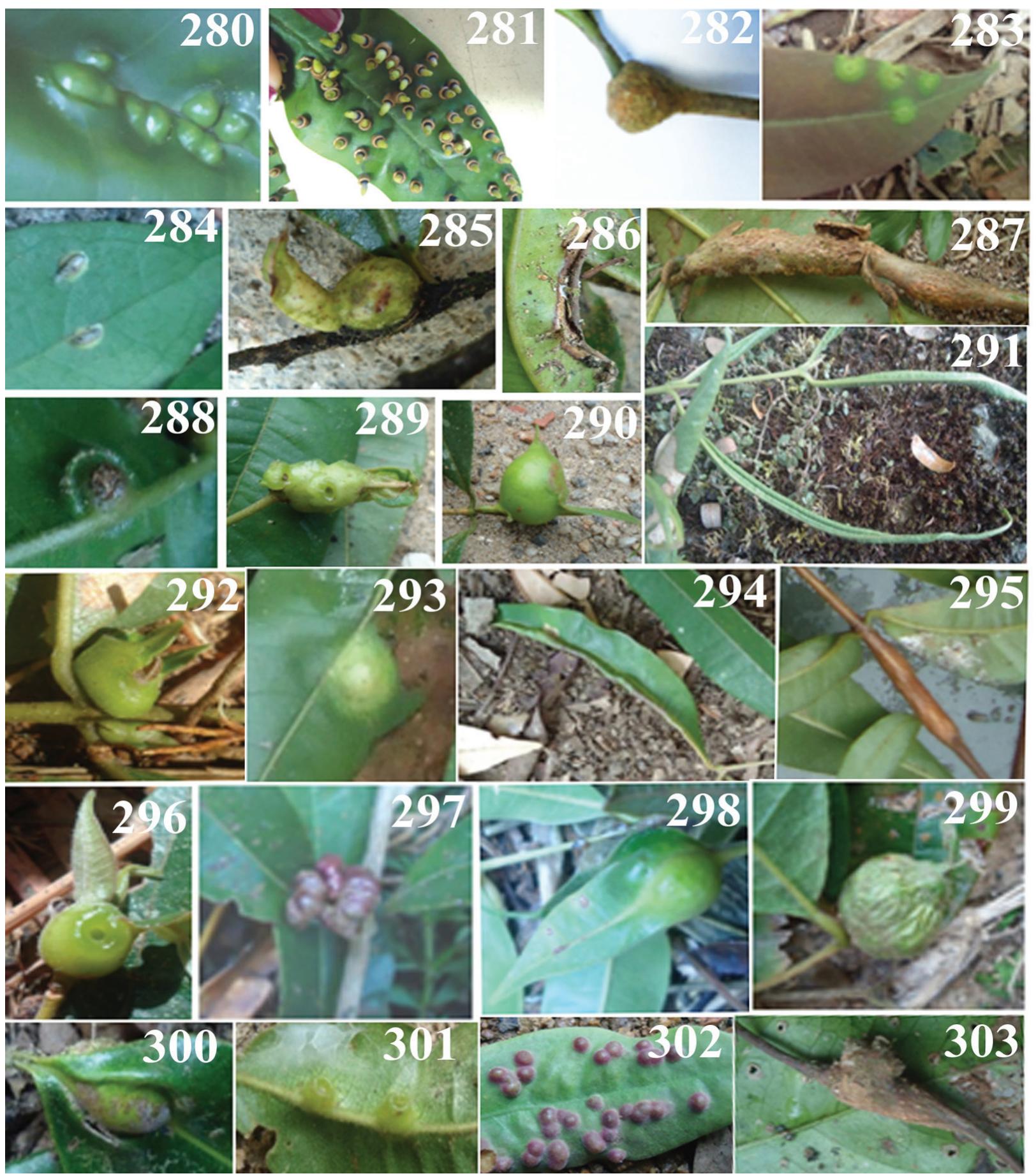

Figure 280-303 - Insect galls of the Parque Nacional do Itatiaia (Southeast Region, Brazil), on Myrtaceae, 280, Calyptranthes sp., globoid leaf gall, 281-282, Eugenia buchosiifolia, 281, cylindrical leaf gall, 282, globoid bud gall, 283, E. schottiana, discoid leaf gall, 284, E. uniflora, discoid leaf gall, 285-287, Eugenia sp., 285, globoid bud gall, 286, marginal leaf roll, 287, fusiform stem gall, 288-291, Myrcia splendens, 288, globoid leaf gall, 289, globoid bud gall, 290, globoid bud and leaf gall, 291, leaf roll gall, 292-293, Myrcia cf. splendens, 292, globoid bud gall, 293, globoid leaf gall, 294-301, M. sylvatica, 294, marginal leaf roll, 295, fusiform stem gall, 296, globoid bud and leaf gall, 297, globoid bud gall, 298, globoid bud and leaf basis gall, 299, globoid bud gall, 300, globoid leaf petiole and midvein gall, 301, globoid leaf midvein gall, 302, Myrcia sp. 1, globoid leaf gall, 303, Myrcia sp. 2, fusiform stem gall. 
Neea oppositifolia Ruiz and Pav. (native species, $\mathrm{NE}$ ) (first record in Southeast Region and in Atlantic forest $)(n=4)$

Gall (Fig. 308): on bud, globoid, green, glabrous, and one-chambered. Galler: Asphondylia sp. (Cecidomyiidae) (04 pupae, 01 larva). Parasitoids: Hymenoptera (05 pupae, 21 adults). Paths: Cachoeira Véu da Noiva, 17/III/2014; Três Picos, 11/XI/2014; Asa de Hermes; 16/IX/2015.

Gall (Fig. 309): on leaf, globoid, green or yellow, glabrous, and one-chambered. Galler: Shizomyiina (Cecidomyiidae) (07 males). Parasitoids: 03 Hymenoptera (02 pupae, 02 adults). Paths: Três Picos, 13/V/2014, 11/XI/2014; Lago Azul, 18/III/ 2014; Cachoeira Véu da Noiva, 17/ III/2014; Donati-Simon 2, 08/IX/2014; BR-485, 12/XI/2014; Barbosa Rodrigues-Mirante do Último Adeus, 09/XII/2014; Cachoeira do Pitu, 25/ II/2015; Cachoeira Poranga, 07/IV/2014.

Gall (Fig. 310): on stem, globoid, green, glabrous, and one-chambered. Galler: Cecidomyiidae (06 larvae). Parasitoids: Hymenoptera (02 larvae, 06 pupae). Inquilines: Lepidoptera (01 catterpilar). Path: Travessia Ruy Braga, 08/XII/2014, 17/III/2015, 16/III/2015, 14/ IV/2015, 15/IX/2015.

Gall (Fig. 311): on stem, globoid, green, and glabrous. Galler: Bruggmannia sp. (Cecidomyiidae) (01 larva). Path: Cachoeira Itaporani, 12/IV/2015.

No previous gall records on this plant species. Neea Ruiz and Pav. sp. (native genus) $(n=1)$

Gall (Fig. 312): on leaf, globoid, yellow, with white trichomes, and one-chambered. Galler: not determined (empty galls). Path: Três Picos, 11/ $\mathrm{XI} / 2014$.

Previous gall records on this genus: 1) in Atlantic forest: on Neea sp. - Kieffer 1913 (n=7/ three in RJ, two in $\mathrm{SC}$, one in $\mathrm{RJ}, \mathrm{SC}$ and $\mathrm{MG}$ ), Maia 2013b (n=2/São Tomé das Letras/MG), Maia 2014 (n=1/Itamonte/MG), 2) in Amazonian Forest: on Neea sp. - Kieffer 1913 (n=1/AM), 3) in Cerrado: on Neea sp. - Kieffer 1913 (n=1/MG), on Neea theifera Oerst - Gonçalves-Alvim and Fernandes 2011 ( $n=1 / /$ Três Marias/MG).

\section{ONAGRACEAE $(\mathrm{N}=1)$}

Ludwigia L. sp. (native genus) ( $\mathrm{n}=1)$

Gall (Fig. 313): on bud, fusiform, green, with whittish pubescence. Galler: Cecidomyiidae (01 larva). Parasitoids: Hymenoptera (01 pupa). Path: Três Picos, 11/XI/2014.

Previous gall records on undetermined species of this plant genus: 1) in Atlantic forest - Maia et al. 2008 ( $\mathrm{n}=1 /$ Bertioga/SP), 2) in Caatinga - Tavares $1918(n=1 /$ Fortaleza/CE $)$.

\section{ORCHIDACEAE $(\mathrm{N}=1)$}

\section{Not determined $(\mathrm{n}=1)$}

Gall (Fig. 314): on leaf, conical, pedunculated, green, and thorny. Galler: not determined. Parasitoids: Hymenoptera (01 pupa). Path: Ecoarte-Lago Azul, 08/IV/2014.

Previous gall records on this plant family: 1) in Atlantic forest - Bregonci et al. $2010(n=1 /$ Guarapari/ES), Kraus and Tanoe 1999 (n=1/São Paulo), Coelho et al. 2013a (n=2/Itatiaia/altitude field) and 2) no bioma data - Gagné 1994 (n=2/ Brazil).

\section{PIPERACEAE $(\mathrm{N}=20)$}

Piper aduncum L. (native species, NE) $(\mathrm{n}=1)$

Gall (Fig. 315): on bud, fusiform, green, with trichomes, and one-chambered. Galler: not determined (empty galls). Path: Hotel Donati, 04/ VIII/2014.

Previous gall records on this plant species: no biome data - Mendonça et al. 2014 ( $n=4 / R S)$.

Piper arboreum Aubl. (native species, NE) $(n=1)$

Gall (Fig. 316): on bud, conical, red, hairy, with white trichomes, and one-chambered. Galler: not determined. Path: Viúva Hansen, 10/IX/2014.

Previous gall records on this plant species: 1) in Atlantic forest - Maia et al. 2008 ( $n=1 /$ Bertioga/ 
SP), Maia et al. 2014 ( $n=2 /$ Santa Teresa/ES), 2) in Cerrado - Araújo et al. 2011 ( $\mathrm{n}=1 /$ Pirineus/GO).

Piper chimonanthifolium Kunth (endemic in Brazil, Cerrado and Atlantic forest) (NE) (n=1)

Gall (Fig. 317): on leaf, globoid, green, with green trichomes. Galler: not determined. Path: Casa 33, 16/VI/2015.

No previous gall records on this plant species. Piper cf. dilatatum Rich. (native species, NE) $(\mathrm{n}=3)$

Gall (Fig. 318): on leaf petiole, fusiform, green, glabrous, and multichambered. Galler: Contarinia sp. (Cecidomyiidae) (05 larvae). Paths: Cachoeira Itaporani, 12/V/2014; Travessia Ruy Braga, 08/ VII/2014.

Gall (Fig. 319): on leaf, globoid, green, with white trichomes, and one-chambered. Galler: Cecidomyiidae (03 larvae). Paths: Cachoeira Itaporani, 17 /III/2014, 12/V/2014; Casa 16, 07/ VII/2014.

Gall (Fig. 320): on leaf petiole and stem, fusiform, green, glabrous, and one-chambered. Galler: not determined. Parasitoids: Hymenoptera (06 adults). Paths: Travessia Ruy Braga, 04/ VIII/2014, 10-12/XI/2014; Viúva Hansen, 10/ IX/2014; BR-485, 28/I/2015; Casa 33, 16/VI/2015.

Previous gall records on this plant species: no biome data - Mendonça et al. 2014 (n=4/RS).

Piper marginatum Jacq. (native species, NE) $(n=2)$

Gall (Fig. 321): on leaf petiole, fusiform, green, with trichomes, and one-chambered. Galler: Lepidoptera (01 catterpilar). Path: Travessia Ruy Braga, 04/VIII/2014.

Gall(Fig.322): onleaf, globoid, green, glabrous, and one-chambered. Galler: Cecidomyiidae (02 pupae). Inquilines: Thysanoptera (01 adult). Path: Três Picos, 11/XI/2014.

No previous gall records on this plant species. Piper richardiifolium Kunth (endemic in Brazil, Caatinga, Cerrado, Amazonian and Atlantic forest, $\mathrm{NE})(\mathrm{n}=1)$
Gall (Fig. 323): on leaf, globoid, green, glabrous, and one-chambered. Galler: Cecidomyiidae (01 larva, 01 pupa). Path: Cachoeira do Pitu, 25/II/2015.

No previous gall records on this plant species. Piper tuberculatum Jacq. (native species, NE) $(\mathrm{n}=3)$

Gall (Fig. 324): on bud, globoid, brown, glabrous, and multichambered. Galler: Zalepidota sp. (Cecidomyiidae) (02 pupal exuviae, 02 males). Parasitoids: Hymenoptera (19 adults). Inquilines: Sciaridae (18 adults). Paths: Donati-Simon 2, 08/ IX/2014; Cachoeira Poranga, 10/IX/2014; Barbosa Rodrigues-Mirante do Último Adeus, 09/XII/2014; BR-485, 28/I/2015, 23/II/2015.

Gall (Fig. 325): on leaf, discoid, green, glabrous, and one-chambered. Galler: not determined. Parasitoids: Hymenoptera (01 adult). Paths: Lago Azul, 18/III/ 2014; Cachoeira Poranga, 07/IV/2014; Ecoarte-Lago Azul, 08/IV/2014; Casa 16, 07/VII/2014; Viúva Hansen, 10/IX/2014; Donati-Simon 2, 08/IX/2014; Barbosa RodriguesMirante do Último Adeus, 09/XII/2014; Barbosa Rodrigues (obelisk), 10/XII/2014; Cachoeira do Pitu, 25/II/2015.

Gall (Fig. 326): on stem, fusiform, green, glabrous, and one-chambered. Galler: not determined (empty gall). Paths: BR-485, 28/I/2015; Travessia Ruy Braga, 114//IV/2015.

No previous gall records on this plant species. Piper L. sp.1 (native genus) ( $\mathrm{n}=2$ )

Gall (Fig. 327): on stem and bud, globoid, green, glabrous, and multichambered. Galler: Cecidomyiidae (01 larva by chamber, 03 young larvae). Inquilines: Coleoptera (01 larva). Paths: Donati-Simon 2, 08/IX/2014; Viúva Hansen, 10/ IX/2014.

Gall (Fig. 328): on stem, globoid, brown, glabrous, and one-chambered. Galler: Lepidoptera (01 catterpilar). Paths: Lago Azul, 18/III/ 2014; Cachoeira Poranga, 07/IV/2014, Cachoeira Véu da 
Noiva, 17/III/2014; Casa 33, 16/VI/2015, BR-485, 23/II/2015, 26/I/2015.

Piper L. sp.2 ( $\mathrm{n}=1)$

Gall (Fig. 329): on bud and leaf, conical, green or red, glabrous, and one-chambered. Galler: not determined (empty galls) (Fig.). Paths: Cachoeira Véu da Noiva, 17/III/2014; Donati-Simon 2, 08/ IX/2014; Viúva Hansen, 10/IX/2014; Barbosa Rodrigues-Mirante do Último Adeus, 09/XII/2014; Cachoeira do Pitu, 25/II/2015.

Piper L. sp. $3(\mathrm{n}=2)$

Gall (Fig. 330): on bud, globoid, brown, glabrous, and multichambered. Galler: not determined (empty gall). Path: Cachoeira Poranga, 07/IV/2014.

Gall (Fig. 331): on bud, cylindrical, brown, glabrous, and one-chambered. Galler: not determined (empty gall). Path: Viúva Hansen, 10/ IX/2014.

Piper L. sp. $4(\mathrm{n}=1)$

Gall (Fig. 332): on stem, fusiform, green, glabrous, and multichambered. Galler: Cecidomyiidae (01 young larva). Path: Três Picos, 13/V/2014.

Piper L sp. 5 ( $\mathrm{n}=1)$

Gall (Fig. 333): on bud, globoid, brown, glabrous, and one-chambered. Galler: Cecidomyiidae (01 young larva). Path: Três Picos, 13/V/2014.

Previous gall records on undetermined species of Piper L.: 1) in Atlantic forest - Rübsaamen 1908 (n=1/Rio de Janeiro/RJ), Maia 2014 (n=3/ Itamonte/MG), 2) in Amazonian forest - Araújo et al. 2012 (n=1/Oriximiná/PA), 3) in Cerrado - Maia and Fernandes 2004 (n=2/Serra de São José/MG), 4) in Pampa - Kieffer 1913 (n=1/São Leopoldo/ RS).

\section{Not determined $(n=1)$}

Gall (Fig. 334): on leaf, globoid, green, with green pubescence, and one-chambered. Galler: Cecidomyiidae (01 young larva). Path: Cachoeira Véu da Noiva, 17/III/2014.
POLYPODIACEAE $(\mathrm{N}=1)$

Niphidium crassifolium (L.) Lellinger (native species, NE) ( $\mathrm{n}=1)$

Gall (Fig. 335): on leaf, globoid adaxially, conical abaxially, green, micropubescent abaxially and opened, one-chambered. Galler: Coccoidea, Hemiptera (15 adults). Parasitoids: Hymenoptera (01 adult). Paths: Cachoeira Poranga, 07/IV/2014, 24/II/2015; BR-485, 12/XI/2014, 28/I/2015; Barbosa Rodrigues-Mirante do Último Adeus, 09/XII/2014; Barbosa Rodrigues (obelisk), 10/ XII/2014; Travessia Ruy Braga, 24/02/2015, 16/ III/2015.

Previous gall records on this plant species: Houard 1933 (Brazil)

\section{PRIMULACEAE $(\mathrm{N}=9)$}

Cybianthus Mart. sp. (native genus) ( $\mathrm{n}=2$ )

Gall (Fig. 336): on bud, fusiform, brown, glabrous, and one-chambered. Galler: Lepidoptera. Paths: Água Branca-Ruy Braga, 18/VI/2014; Travessia Serra Negra, 14-15/X/2014; Morro do Couto, 13/X/2014; Pedra do Camelo, 14/IX/2015.

Gall (Fig. 337): on stem, fusiform, brown, and glabrous. Galler: not determined. Path: Travessia Serra Negra, 14-15/X/2014.

Previous gall records on this plant genus: 1) in Amazonian forest: on Cybianthus sp.- Santos et al. 2011b ( $\mathrm{n}=1 /$ six municipalities/PE), on Cybianthus guyanensis (A. DC.) Miq. - Araújo et al. 2012 ( $\mathrm{n}=1$ / Oriximiná/PA), 2) in Cerrado: on Cybianthus sp. - Gonçalves-Alvim and Fernandes 2001 ( $\mathrm{n}=2 /$ Três Marias/MG).

Myrsine coriacea (Sw.) R.Br. ex Roem. and Schult. (=Rapanea ferruginea (Ruiz and Pav.) Mez. (native species, NE) ( $\mathrm{n}=1)$

Gall (no fig.): on stem, fusiform, brown, glabrous, and one-chambered. Galler: not determined. Path: Travessia Ruy Braga, 15/ IX/2015. 

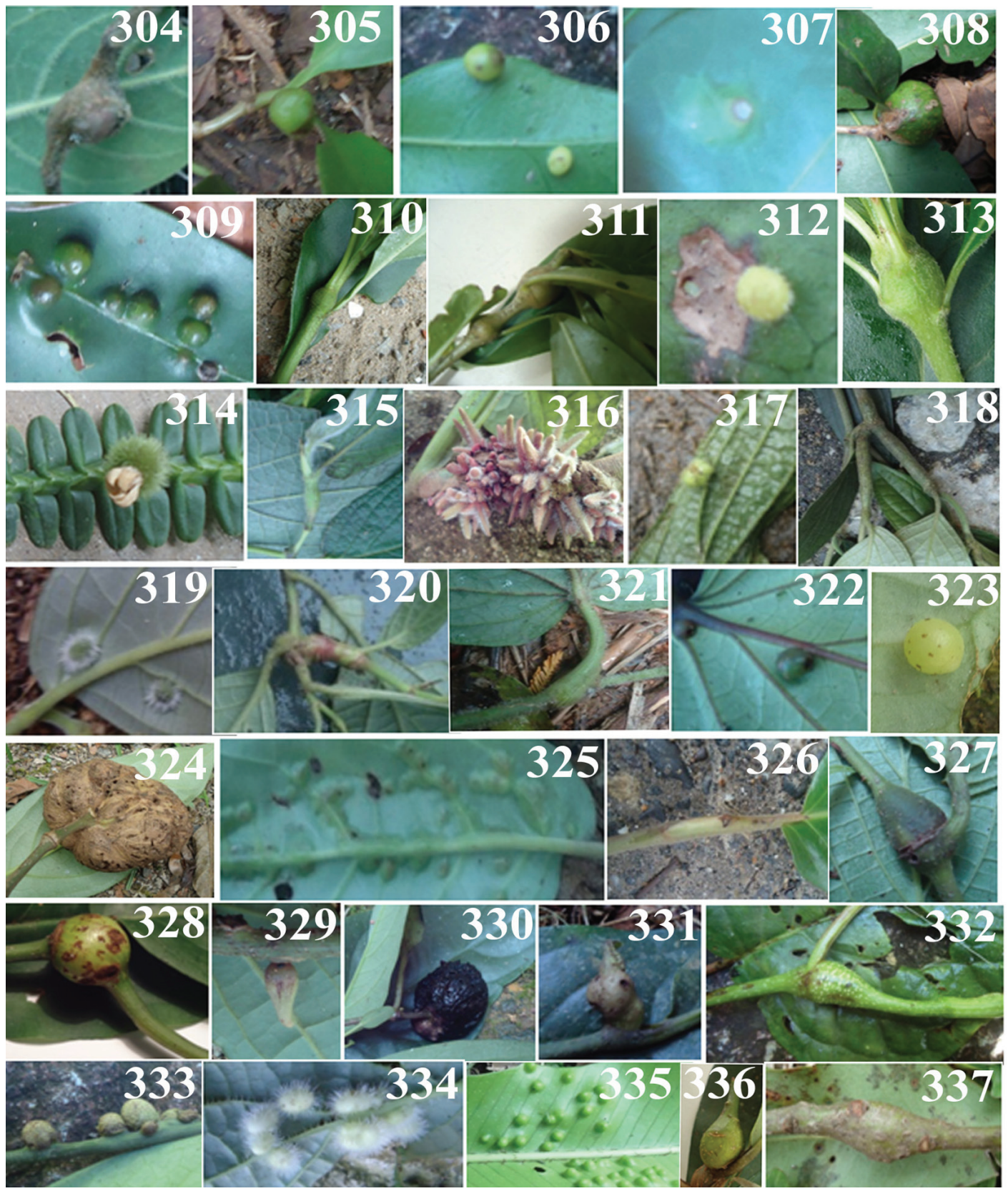

Figure 304-337 - Insect galls of the Parque Nacional do Itatiaia (Southeast Region, Brazil), 304-312, on Nyctaginaceae, 304, Bougainvillea spectabilis, globoid stem gall, 305-307, Guapira sp., 305, globoid bud and leaf gall, 306, globoid leaf gall, 307, discoid leaf gall, 308-311, Neea oppositifolia, 308, globoid bud gall, 309, globoid leaf gall, 310, globoid stem gall, 311, globoid stem gall, 312, Neea sp. , globoid leaf gall, 313, on Onagraceae, Ludwigia sp., fusiform bud gall, 314, on Orchidaceae sp., conical leaf gall, 315-334, on Piperaceae, 315, Piper aduncum, conical bud gall, 316, P. arboreum, conical bud gall, 317, P. chimonanthifolium, globoid leaf gall, 318-320, Piper cf. dilatatum, 318, fusiform leaf petiole gall, 319, globoid leaf gall, 320, fusiform stem and leaf petiole gall, 321-322, P. marginatum, 321, fusiform leaf petiole gall, 322, globoid leaf gall, 323, P. richardiifolium, globoid leaf gall, 324-326, P. tuberculatum, 324, globoid bud gall, 325, discoid leaf gall, 326, fusiform stem gall, 327-328, Piper sp. 1, 327, globoid stem and bud gall, 328, globoid stem gall, 329, Piper sp. 2, conical bud and leaf gall, 330-331, Piper sp. 3, 330, globoid bud gall, 331, cylindrical bud gall, 332, Piper sp. 4, fusiform stem gall, 333, Piper sp. 5, globoid bud gall, 334, Piperaceae sp.1, globoid leaf gall, 335, on Polypodiaceae, Niphidium crassifolium, conical leaf gall, 336-337, on Primulaceae, Cybianthus sp., 336, fusiform bud gall, 337 , fusiform stem gall. 
Previous gall records on this plant species: 1) in Atlantic forest, as Rapanea ferruginea - Maia et al. 2008 ( $n=2 / B e r t i o g a / S P)$, Toma and Mendonça 2013 (n=1/São Francisco de Paula/RS).

Myrsine lineata (Mez) Imkhan. (endemic in Brazil, Atlantic forest, NE) $(n=1)$

Gall(Fig. 338): on leaf, discoid, green, glabrous, and one-chambered. Galler: Cecidomyiidae (06 larvae). Path: Travessia Ruy Braga, 16/III/2015.

No previous gall records on this plant species. Myrsine parvula (Mez) Otegui (native species, $\mathrm{NE})$ (first record in altitude fields) $(\mathrm{n}=1)$

Gall (Fig. 339): on stem, globoid, reddish, glabrous, and one-chambered. Galler: Lepidoptera (03 catterpilars, 01 adult). Inquilines: Curculionidae (01 adult). Paths: Prateleiras, 09/IV/2014; Morro do Couto, 13/V/2014; Travessia Serra Negra, 14-15/X/2014; Travessia Ruy Braga, 14/IV/2015, 15/IX/2015; Asa de Hermes, 16/IX/2015; Posto Marcão-Abrigo Rebouças, 13/V/2015.

No previous gall records on this plant species. Myrsine L. sp. 1 (native genus) $(\mathrm{n}=3)$

Gall (Fig. 340): on leaf, discoid, reddish peripherally and green centrally, glabrous, and onechambered. Galler: Contarinia sp. (Cecidomyiidae) (03 larvae). Parasitoids: Hymenoptera (01 pupa). Paths: Água Branca-Ruy Braga, 18/VI/2014; Travessia Ruy Braga, 17/III/2015, 14/IV/2015, 15/ IX/2015.

Gall (Fig. 341): on apical and lateral bud, conical, green or brown, glabrous, and onechambered. Galler: Lepidoptera (01 caterpillar). Path: Travessia Ruy Braga, 14/IV/2015.

Gall (Fig. 342): on bud, rosette, green, glabrous, and one-chambered. Galler: not determined. Path: Travessia Ruy Braga, 14/IV/2015.

Myrsine L sp. 2 ( $\mathrm{n}=1$ )

Gall (Fig. 343): on flower bud, conical, reddish, glabrous, and one-chambered. Galler: Clinodiplosis sp. (Cecidomyiidae) (02 larvae). Path: Travessia Ruy Braga, 04/VIII/2014.
Previous gall records on undetermined species of Myrsine L.: 1) in Atlantic forest - Maia and Oliveira 2010 ( $\mathrm{n}=1 /$ Angra dos Reis/RJ), Maia et al. $2014(\mathrm{n}=2 /$ Santa Teresa/ES).

\section{PROTEACEAE $(\mathrm{N}=3)$}

Roupala montana Aubl. var. montana (native species, NE) ( $\mathrm{n}=3)$

Gall (Fig. 344): on bud and stem, fusiform, brown, unilateral, glabrous, and one-chambered or multichambered. Galler: Lopesiini(Cecidomyiidae) (02 larvae). Parasitoids: Hymenoptera (adult fragments). Paths: Asa de Hermes, 16/IX/2015; Travessia Ruy Braga, 13/V/2015.

Gall (Fig. 345): on stem, globoid, brown, glabrous, and one-chambered. Galler: Lepidoptera. Paths: Prateleiras, 09/IV/2014; Morro do Couto, 13/V/2014; Travessia Serra Negra, 14-15/X/2014; Travessia Ruy Braga, 14/IV/2015, 13/V/2015.

Gall (Fig. 346): on bud, globoid, green, glabrous, and one-chambered. Galler: Lepidoptera. Paths: Prateleiras, 09/IV/2014; Pedra da Maçã, Tartaruga and Assentada, 27/I/2015.

Previous gall records: 1) in Atlantic forest Gonçalves-Alvim and Fernandes 2001 ( $n=1 /$ Três Marias/MG), Toma and Mendonça 2013 (n=1/São Francisco de Paula/RS), Maia 2014 (n=2/ Itamonte/ MG), Maia 2013b ( $\mathrm{n}=1 /$ São Tomé das Letras/MG), 2) in Cerrado - Araújo et al. $2011 b$ ( $n=1 / G O)$, Saito and Urso-Guimarães 2013 (n=2/Jataí/MG), Araújo et al. $2015(\mathrm{n}=6 / \mathrm{GO})$.

PTERIDACEAE $(\mathrm{N}=1)$

Adiantum latifolium Lam. (native species, NE) $(\mathrm{n}=1)$

Gall (Fig. 347): on bud, globoid, brown, glabrous, and one-chambered. Galler: not determined. Path: Donati-Simon 2, 08/IX/2014.

No previous gall records on this plant species and genus. 


\section{ROSACEAE $(\mathrm{N}=2)$}

Prunus myrtifolia (L.) Urb. (native species, NE) $(\mathrm{n}=1)$

Gall (Fig. 348): on stem, fusiform, brown, glabrous, and one-chambered. Galler: Cecidomyiidae (01 young larva). Parasitoids: Hymenoptera (04 pupae, 01 adult). Path: Travessia Ruy Braga, 08/XII/2014.

No previous gall records on this plant species. Rubus rosifolius $\mathrm{Sm}$. (exotic species) $(\mathrm{n}=1)$

Gall (Fig. 349): on leaf, marginal roll, green, glabrous, and one-chambered. Galler: not determined. Inquilines: Lepidoptera (01 pupa). Path: Ecoarte-Lago Azul, 08/IV/2014.

Previous gall records on this plant genus: 1) in Atlantic forest: on Rubus sp. -Maia et al. 2014 (n=1/ Santa Teresa/ES), on Rubus erythrocladus Mart Toma and Mendonça 2013 (n=1/São Francisco de Paula/RS), 2) in Cerrado: on Rubus sp. -Tavares 1908 (n=1/ Serra do Caraça/MG), 3) no biome data: on $R$. erythrocladosMart. Ex Hook.f. - Mendonça et al. $2014(\mathrm{n}=4 / \mathrm{RS})$.

\section{RUBIACEAE $(\mathrm{N}=16)$}

Borreria tenera DC. (native species, NE) (first record in Southeast Region and Atlantic forest) $(\mathrm{n}=1)$

Gall (Fig. 350): on stem, globoid, green, glabrous, and one-chambered. Galler: Cecidomyiidae (02 larvae). Parasitoids: Hymenoptera (01 larva). Paths: Água Branca-Ruy Braga, 18/VI/2014; Travessia Serra Negra, 14-15/ X/2014; Travessia Ruy Braga, 16/III/2015.

No previous gall records on this plant species. Borreria G. Mey sp. (native genus) $(\mathrm{n}=1)$

Gall (Fig. 351): on stem, fusiform, brown, glabrous, and one-chambered. Galler: Neolasioptera sp. (Cecidomyiidae) (02 larvae). Parasitoids: Hymenoptera (02 larvae). Path: Três Picos, 08/IX/2014.
Previous records on Borreria spp.: 1) in Atlantic forest: on Borreria sp. - Rübsaamen 1905 ( $\mathrm{n}=1 /$ Rio de Janeiro/RJ), on B. verticillata (L.) Meyer - Maia 2013a (n=1/Maricá, Arraial do Cabo and Carapebus/RJ), on Borreria cf. ocymifolia (Willd. Ex Roem. and Schult) Bacigalupe and E.L. Cabral - Maia et al. 2008 ( $n=1 /$ Bertioga/SP), on Borreria cf. brachystemonoides - Rodrigues et al. 2014 (n=1/Mangaratiba/RJ).

Cordiera myrciifolia (K.Schum.) C.H.Perss. and Delprete (native species, NE) $(n=1)$

Gall (Fig. 352): on bud, globoid, with crests, green, glabrous, and one-chambered. Galler: Lepidoptera (02 catterpilars). Path: BR-485, 12/ $\mathrm{XI} / 2014$.

No previous gall on this plant species. Previous gall records on this plant genus: in Cerrado: on Cordiera concolor (Cham.) Kuntze - Carneiro et al. 2009b ( $\mathrm{n}=1 /$ Serra do Espinhaço/MG).

Ixora L. sp. (native genus) $(\mathrm{n}=1)$

Gall (Fig. 353): on leaf, globoid, green, hairy, and one-chambered. Galler: Cecidomyiidae (01 pupa). Path: Cachoeira Poranga, 07/IV/2014.

No previous gall records on this plant genus. Psychotria leiocarpa Cham. and Schltdl. (native species, NE) ( $\mathrm{n}=1)$

Gall (Fig. 354): on leaf, conical, green, glabrous, and one-chambered. Galler: not determined (empty galls). Path: Travessia Ruy Braga, 08/XII/2014.

Previous gall records on this plant species: in Atlantic forest - Lima et al. 2000 (same gall morphotype/Bertioga/SP).

Psychotria L. sp.1 (native genus) $(\mathrm{n}=4)$

Gall (Fig. 355): on bud, stem and leaf petiole, fusiform, reddish or green, glabrous, and onechambered. Galler: Cecidomyiidae (02 young larvae). Parasitoids: Hymenoptera (07 adults). Paths: Três Picos, 10/IX/2014, 11/XI/2014; BR-485, 12/XI/2014; Travessia Ruy Braga, 17/ III/2015; 14/IV/2015; Casa 33, 16/VI/2015. 
Gall (Fig. 356): on leaf, globoid, green, succulent, glabrous, and one-chambered. Galler: Cecidomyiidae (02 larvae, 01 pupa). Parasitoids: Hymenoptera (01 pupa, 03 adults). Paths: Cachoeira Véu da Noiva, 17/III/2014; Cachoeira Itaporani, 12/V/2014; Três Picos 13/V/2014, 10/IX/2014; Água Branca-Ruy Braga, 18/VI/2014; Travessia Serra Negra, 14-15/X/2014 (01 pupa); Três Picos 11/XI/2014; BR-485, 12/XI/2014; Travessia Ruy Braga, 18/VI/2014, 24/II/2015, 17/III/2015, 14/ IV/2015.

Similar gall recorded by Maia 2014 in the PNI (Brejo da Lapa).

Gall (Fig. 357): on leaf, marginal roll, green, glabrous, and one-chambered. Galler: not determined (empty galls). Path: Água Branca-Ruy Braga, 18/VI/2014.

Gall (Fig. 358): on leaf, globoid, yellowish, with trichomes, and one-chambered. Galler: not determined (empty galls). Path: Travessia Ruy Braga, 24/II/2015.

Psychotria L. sp.2 ( $\mathrm{n}=1)$

Gall (Fig. 359): on bud, globoid, brown, hairy, and one-chambered. Galler: Lepidoptera (fragments). Path: Três Picos, 08/IX/2014.

Previous gall records on undetermined species of Psychotria L.: in Atlantic forest - Tavares 1922 ( $\mathrm{n}=1 /$ Friburgo/RJ), Maia et al. 2014 ( $\mathrm{n}=1 /$ Santa Teresa/ES), Maia 2014 (n=1/Itamonte/MG), Maia and Carvalho-Fernandes 2016 (n=1/São Francisco de Itabapoana/RJ).

Randia L. sp. (native genus) ( $\mathrm{n}=1$ )

Gall (Fig. 360): on leaf, fold, green, glabrous, and one-chambered. Galler: Hemiptera. Path: Casa de Pedra, 25/II/2014.

No previous records on this plant genus. Remijia DC. sp. (native genus) (first record in RJ) $(\mathrm{n}=1)$

Gall (Fig. 361): on leaf, globoid, whittish or greenish, hairy, and one-chambered. Galler: Lasiopteridi (Cecidomyiidae) (01 male).
Parasitoids: Hymenoptera (01 larva, 04 adults). Path: Donati-Simon 2, 08/IX/2014.

Previous gall records: in Cerrado: on Remijia ferruginea (A. St.-Hil) DC. - Carneiro et al. 2009b ( $\mathrm{n}=1 /$ Serra do Espinhaço/MG).

Rudgea Salisb. sp. (native genus) $(\mathrm{n}=1)$

Gall (Fig. 362): on leaf, discoid, yellow, glabrous, and one-chambered. Galler: not determined (empty galls). Path: Travessia Ruy Braga, 08/VII/2014.

Previous gall records on this plant genus: in Atlantic forest: on Rudgea parquioides (Cham.) Müll. Arg. - Toma and Mendonça 2013 (n=1/São Francisco de Paula/RS), Mendonça et al. 2014 $(\mathrm{n}=1 / \mathrm{RS})$.

Sabicea Aubl. sp. (native genus) $(\mathrm{n}=1)$

Gall (Fig. 363): on leaf, globoid, yellow, hairy, and one-chambered. Galler: Cecidomyiidae (02 larvae). Path: Travessia Ruy Braga, 04/VIII/2014.

Previous records on this plant genus: in Atlantic forest: on Sabicae brasiliensis Wernham Maia 2013b (n=1/São Tomé das Letras/MG).

Sipanea Aubl. sp.1 (native genus) (first record in $\mathrm{RJ})(\mathrm{n}=1)$

Gall (Fig. 364): on stem, globoid, green, and micropubescent. Galler: Cecidomyiidae (01 young larva). Paths: Cachoeira Itaporani, 17/III/2014; Três Picos, 13/V/2014.

Sipanea Aubl. sp. $2(\mathrm{n}=1)$

Gall (Fig. 365): on leaf, globoid, green, with green trichomes, and one-chambered. Galler: not determined (empty galls) (Fig.). Paths: Cachoeira Itaporani, 17/III/2014, 12/V/2014; Travessia Serra Negra, 14-15/X/2014.

Previous gall records on undetermined species of Sipanea Aubl: in Atlantic forest - Maia et al. $2014(\mathrm{n}=1 /$ Santa Teresa/ES).

\section{SALICACEAE $(\mathrm{N}=3)$}

Casearia aculeata Jacq. (native species, NE) (first record in RJ and altitude fields) $(n=1)$ 

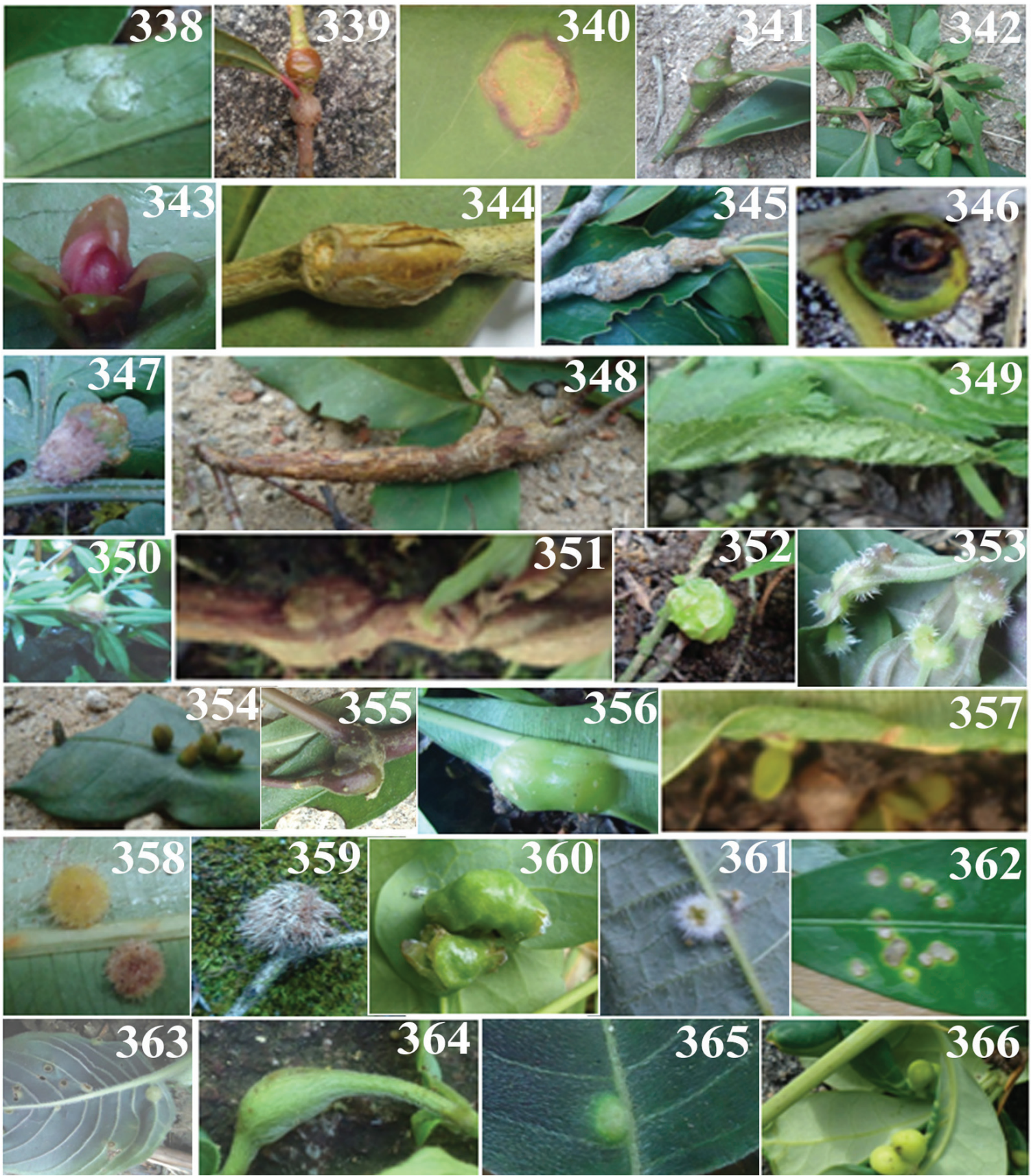

Figure 338-366 - Insect galls of the Parque Nacional do Itatiaia (Southeast Region, Brazil), 338-343, on Primulaceae, 338, Myrsine lineata, discoid leaf gall, 339, M. parvula, globoid stem gall, 340-342, Myrsine sp. 1, 340, discoid leaf gall, 341, conical bud gall, 342, rosette bud gall, 343, Myrsine sp. 2, conical flower bud gall, 344-346, on Proteaceae, Roupala montana montana, 344, fusiform bud and stem gall, 345, globoid stem gall, 346, globoid bud gall, 347, on Pteridaceae, Adiantum latifolium, globoid bud gall, 348-349, on Rosaceae, 348, Prunus myrtifolia, fusiform stem gall, 349, Rubus rosifolius, marginal leaf roll, 350-365, on Rubiaceae, 350, Borreria tenera, globoid stem gall, 351, Borreria sp., fusiform stem gall, 352, Cordiera myrciifolia, globoid bud gall, 353, Ixora sp., globoid leaf gall, 354, Psychotria leiocarpa, conical leaf gall, 355-358, Psychotria sp.1, 355, fusiform bud, stem and leaf petiole gall, 356, globoid leaf gall, 357, marginal leaf roll, 358, globoid leaf gall, 359, Psychotria sp. 2, globoid bud gall, 360, Randia sp., leaf fold, 361, Remijia sp., globoid leaf gall, 362, Rudgea sp., discoid leaf gall, 363, Sabicea sp., globoid leaf gall, 364, Sipanea sp. 1, globoid stem gall, 365, Sipanea sp. 2, globoid leaf gall, 366, on Salicaceae, Casearia aculeata, globoid leaf gall. 
Gall (Fig. 366): on leaf, globoid, green, glabrous, and one-chambered. Galler: Asphondylia sp. (Cecidomyiidae) (1 pupa). Inquilines: Coccoidea (Hemiptera). Path: Posto MarcãoAbrigo Rebouças, 24/II/2014.

Previous gall records on this plant species: in Atlantic forest: on Casearia cf. aculeata Fernandes and Negreiros 2006 (n=1/Aimorés/MG) Casearia arborea (Rich.) Urb. (native species, NE) $(\mathrm{n}=1)$

Gall (Fig. 367): on leaf vein, fusiform, green, glabrous, and one-chambered. Galler not determined. Path: Travessia Ruy Braga, 08/VII/201.

Previous gall records on this plant species: 1) in Atlantic forest - Maia et al. 2014 ( $n=1 /$ Santa Teresa/ES), 2) in Amazonian forest - Almada and Fernandes 2011 ( $\mathrm{n}=2 /$ Oriximiná/PA).

Casearia sylvestris $\mathrm{Sw}$. (native species, NE) $(\mathrm{n}=1)$

Gall (Fig. 368): on stem, fusiform, green, glabrous, and one-chambered. Galler: not determined. Path: BR-485, 09/XII/2014.

Previous gall records on this plant species: 1) in Atlantic forest - Santos et al. $2012(n=2 /$ PE), 2) in Amazonian forest - Maia 2011 ( $n=1 /$ Oriximiná/PA), 3) in Cerrado - Araújo et al. 2011 ( $\mathrm{n}=2 /$ Pirineus/GO), 4) in Caatinga-Cerrado - Luz et al. 2012 ( $\mathrm{n}=1 /$ Januária/MG), 5) no data biome Mendonça et al. 2014 ( $n=3 / R S)$.

SANTALACEAE $(\mathrm{N}=2)$

Phoradendron Nutt. sp. (native genus) ( $\mathrm{n}=1$ )

Gall (Fig. 369): on leaf, globoid, brown, glabrous, and two-chambered. Galler: Cecidomyiidae (01 young larva). Path: Travessia Ruy Braga, 10 -12/XI/2014.

Previous gall records on this plant genus: 1) in Atlantic forest: on Phoradendron sp. - Maia 2014 ( $\mathrm{n}=1 /$ Santa Teresa/ES), on P. piperoides (Kunth) Trel. - Monteiro et al. 1994 (n=1/Maricá/RJ), 2) in Cerrado: on P.crassifolium (Pohl ex DC) Eichler
- Carneiro et al. 2009b ( $\mathrm{n}=1 /$ Serra do Espinhaço/ $\mathrm{MG})$.

Not determined $(n=1)$

Gall (Fig. 370): on stem, fusiform, brown, and glabrous. Galler: not determined. Path: Casa de Pedra, 25/II/2014.

\section{SAPINDACEAE $(\mathrm{N}=20)$}

Cupania cinerea Poepp. and Endl. (native species, NE) (first record in the Southeast Region and Atlantic forest) $(n=1)$

Gall (Fig. 371): on stem, fusiform, brown, glabrous, and one-chambered. Galler: Cecidomyiidae (01 larva). Path: Centro de Visitantes, 07-08/VII/2014.

No previous gall records on this plant species. Cupania scrobiculata Rich. (native species, NE) (first record in the Southeast Region) $(\mathrm{n}=1)$

Gall (Fig. 372): on leaf, globoid, red or green, with yellow trichomes, and one-chambered. Galler: Cecidomyiidae (03 larvae). Parasitoids: Hymenoptera (01 larva). Path: Donati-Simon 2, 08/IX/2014.

Previous gall records on this plant species: in Amazonian forest - Maia 2011 ( $n=1 /$ Oriximiná/ PA).

Paullinia L. sp. (native genus) $(\mathrm{n}=1)$

Gall (Fig. 373): on stem, unilateral, fusiform, brown, glabrous, and one-chambered. Galler: Cecidomyiidae (01 adult). Path: Travessia Ruy Braga, 10-12/XI/2014.

Previous gall records on undetermined species of Paullinia L.: 1) in Atlantic forest - Maia et al. 2008 (n=6/Bertioga/SP), 2) in Cerrado - Maia and Fernandes 2004 (n=1/Serra de São José/MG).

Serjania communis Cambess. (native species, NE) $(\mathrm{n}=1)$

Gall (Fig. 374): on stem, tendril, leaf petiole, and vein, fusiform, green, micropubescent, and one-chambered. Galler: not determined. Path: BR485, 26/I/2015, 28/I/2015. 
Previous gall records on this plant species: in Atlantic forest - Maia et al. 2008 ( $n=1 /$ Bertioga/ SP).

Serjania deflexa Gardner (native species, NE) $(\mathrm{n}=1)$

Gall (Fig. 375): on stem, tendril, leaf petiole, and vein, fusiform, green, pubescent, and onechambered. Galler: Cecidomyiidae (05 larvae). Paths: Casa 33, 16/VI/2015; BR-485, 28/I/2015.

No previous gall records on this plant species. Serjania glutinosa Radlk. (native species, NE) (first record in RJ) $(n=2)$

Gall (Fig. 376): on leaf vein, fusiform, green, glabrous, and one-chambered. Galler: Cecidomyiidae(01 larva). Parasitoids: Hymenoptera (fragments). Inquilines: Muscomorpha (01 larva). Paths: Hotel Donati, 04/VIII/2014; Travessia Ruy Braga, 10-12/XI/2014.

Gall (Fig. 377): on leaf petiole, tendril and stem, fusiform, green with brown trichomes, and one-chambered. Galler: not determined. Path: Travessia Ruy Braga, 08/VII/2014.

Previous gall records on this plant species: in Atlantic forest - Maia 2013b (n=1/São Tomé das Letras/MG).

Serjania lethalis A.St.-Hil. (native species, NE) $(\mathrm{n}=1)$

Gall (Fig. 378): on bud and stem, fusiform, unilateral, brown, and one-chambered. Galler: not determined (empt galls). Paths: Hotel Donati, 04/ VIII/2014; Donati-Simon 2, 08/IX/2014.

Previous gall records: in Cerrado - Luz et al. 2012 ( $\mathrm{n}=1 /$ Januária/MG), Araújo et al. 2015 (n=1/ $\mathrm{GO} /$ ).

Serjania meridionalis Cambess. (native species, $\mathrm{NE})(\mathrm{n}=2)$

Gall (Fig. 379): on stem, leaf petiole and midvein, fusiform, brown, and one-chambered. Galler not determined (empty galls). Paths: Viúva Hansen, 10/IV/2014; Água Branca-Ruy Braga, 18/ $\mathrm{VI} / 2014$.
Gall (Fig. 380): on bud, globoid, brown, unilateral, and glabrous. Galler: not determined. Path: Travessia Ruy Braga, 08/VII/2014.

Previous gall records on this plant species: 1) in Atlantic forest - Maia 2013b (n=1/São Tomé das Letras/MG), 2) no biome data - Mendonça et al. 2014 (n=3/RS).

Serjania paucidentata DC. (native species, NE) (first record in RJ) ( $\mathrm{n}=5)$

Gall (Fig. 381): on leaf vein, fusiform, green, glabrous, and one-chambered. Galler: Cecidomyiidae (01 larva). Parasitóides: Hymenoptera (fragments). Path: Travessia Ruy Braga, 18/VI/2014, 14/IV/2015.

Gall (Fig. 382): on stem, fusiform, brown, glabrous, and one-chambered. Galler: Lepidoptera. Path: Travessia Ruy Braga, 18/VI/2014, 14/ IV/2015.

Gall (Fig. 383): on leaf petiole, globoid, brown, and glabrous. Galler: Coleoptera (01 larva). Parasitoids: Hymenoptera (fragments). Path: Travessia Ruy Braga, 14/IV/2015.

Gall (Fig. 384): on leaf, marginal roll, reddish, glabrous, and one-chambered. Galler: not determined (empty galls). Path: Travessia Ruy Braga, 14/IV/2015.

Gall (Fig. 385): on leaf, discoid, with central concavity, brown, glabrous, and one-chambered. Galler: not determined. Inquilines: Thysanoptera (02 nimphs). Paths: Água Branca-Ruy Braga, 18/ VI/2014; Travessia Ruy Braga, 14/IV/2015.

No previous record on this plant.

Serjania Mill. sp.1 (native genus) $(\mathrm{n}=1)$

Gall (Fig. 386): on leaf, marginal fold, green or red, irregular, glabrous, and one-chambered. Galler: not determined. Path: Casa de Pedra, 25/ II/2014.

Serjania Mill. sp.2 ( $\mathrm{n}=1)$

Gall (Fig. 387): on stem, globoid, brown, glabrous, and one-chambered. Galler: not determined (empty galls). Paths: Lago Azul, 18/ III/ 2014. 


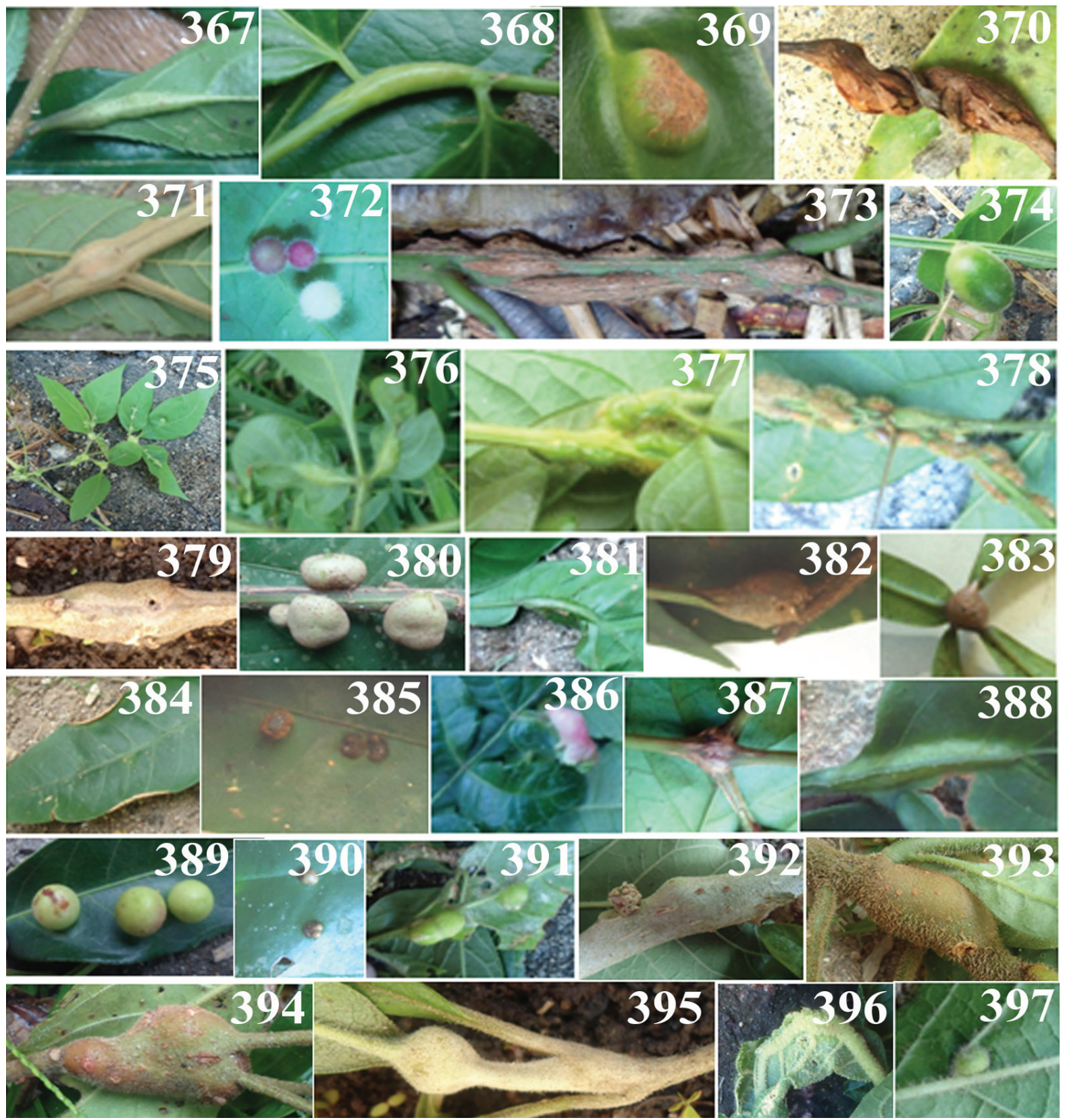

Figure 367-397 - Insect galls of the Parque Nacional do Itatiaia (Southeast Region, Brazil), 367-368, on Salicaceae, Casearia arborea, 367, fusiform leaf vein gall, 368, C. sylvestris, fusiform stem gall, 369-370, on Santalaceae, 369, Phoradendron sp., globoid leaf gall, 370, Santalaceae sp., fusiform stem gall, 371-390, on Sapindaceae, 371, Cupania cinerea, fusiform stem gall, 372, C. scrobiculata, globoid leaf gall, 373, Paullinia sp., fusiform stem gall, 374, Serjania communis, fusiform stem, tendril, leaf petiole and vein gall, 375, S. deflexa, fusiform stem, tendril, leaf petiole and vein gall, 376-377, S. glutinosa, 376, fusiform leaf vein gall, 377, fusiform stem, tendril, leaf petiole, 378, S.lethalis, fusiform bud and stem gall, 379-380, S. meridionalis, 379, fusiform stem, leaf petiole and midvein gall, 380, globoid bud gall, 381-385, S. paucidentata, 381, fusiform leaf vein gall, 382, fusiform stem gall, 383, globoid leaf petiole gall, 384, marginal leaf roll, 385, discoid leaf gall, 386, Serjania sp. 1, marginal leaf roll, 387, Serjania sp. 2, globoid stem gall, 388, Serjania sp. 3, fusiform leaf midvein gall, 389, Serjania sp. 4, globoid leaf gall, 390, Toulicia sp., globoid leaf gall, 391-397, on Solanaceae, 391-392, Acnistus arborescens, 391, globoid leaf gall, 392, fusiform stem gall, 393, Aureliana anonacea, fusiform stem gall, 394, Aureliana sp., fusiform bud and stem gall, 395, Solanum asperum, globoid stem and leaf petiole gall, 396, S. incinellum, fusiform leaf vein gall, 397, S. megalochiton, globoid leaf gall. 
Serjania Mill. sp.3 (n=1)

Gall (Fig. 388): on leaf midvein, fusiform, green, glabrous, and one-chambered. Galler: Cecidomyiidae (01 larva). Parasitoids: Hymenoptera (02 pupae, 02 adults). Paths: Lago Azul, 18/III/2014; Cachoeira Poranga, 07/IV/2014; Travessia Ruy Braga, 10-12/XI/2014; BR-485, 09/ XII/2014, 23/II/2015; Viúva Hansen, 10/IX/2014; Barbosa Rodrigues-Mirante do Último Adeus, 09/ XII/2014.

\section{Serjania Mill. sp.4 ( $\mathrm{n}=1)$}

Gall (Fig. 389): on leaf, globoid, green or red, glabrous, and one-chambered. Galler: Cecidomyiidae (01 larva). Path: Hotel Donati, 04/ VIII/2014.

Previous gall records on undetermined species of Serjania Mill.: 1) in Atlantic forest - Maia et al. 2014 ( $n=2 /$ Santa Teresa/ES), Rodrigues et al. 2014 ( $\mathrm{n}=6 /$ Marambaia/RJ), Maia 2014 (n=1/Itamonte/ MG), Maia 2013b (n=1/São Tomé das Letras/ MG), Maia and Carvalho-Fernandes 2016 (n=7/ São Francisco de Itabapoana/RJ), 2) in Amazonian forest - Rübsaamen 1916 ( $\mathrm{n}=1 / \mathrm{AM})$, Almada and Fernandes 2011 ( $\mathrm{n}=1 /$ Oriximiná/PA), Araújo et al. 2012 ( $\mathrm{n}=1 /$ Oriximiná/PA), 3) in Cerrado - Coelho et al. 2009 ( $n=7 /$ Serra do Cipó/MG), Araújo et al. 2015 ( $\mathrm{n}=7 /$ /Goiás), 4) in Cerrado-Caatinga - Costa et al. 2015 ( $\mathrm{n}=1 /$ Caetité/BA).

Toulicia Aubl. sp. (native genus) $(\mathrm{n}=1)$

Gall (Fig. 390): on leaf, globoid, brown, glabrous, and one-chambered. Galler: not determined. Path: Viúva Hansen, 10/IX/2014.

Previous gall records on this plant genus: in Cerrado: on Toulicia tomentosa Radlk. - UrsoGuimarães and Scareli-Santos 2006 (n=1/Santa Rita do Passa Quatro/MG).

SOLANACEAE $(\mathrm{N}=23)$

Acnistus arborescens (L.) Schltd. (native species, $\mathrm{NE})$ (first record in altitude fields) $(\mathrm{n}=2)$
Gall (Fig. 391): on leaf, globoid, green, with white trichomes, and one-chambered. Galler: Cecidomyiidae (01 larva). Inquilines: Thysanoptera (01 adult and 03 nymphs). Paths: Casa de Pedra, 25/II/2014; Travessia Serra Negra, 14-15/X/2014; Travessia Ruy Braga, 24/II/2015, 17/III/2015; 14/ IV/2015.

Gall (Fig. 392): on stem, fusiform, brown, glabrous, and one-chambered. Galler: not determined. Path: Barbosa Rodrigues-Mirante do Último Adeus, 09/XII/2014.

No previous gall records of galls on this plant. Aureliana anonacea (Sendtn.) I.M.C.Rodrigues and Stehmann (endemic in Brazil, Atlantic forest, NE) $(n=1)$

Gall (Fig. 393): on stem, fusiform, brown, and pubescent. Galler: not determined. Path: Travessia Ruy Braga, 14/IV/2015.

No previous gall records of galls on this plant. Aureliana Sendtn. sp. (native genus) $(\mathrm{n}=1)$

Gall (Fig. 394): on bud and stem, fusiform, green or reddish, glabrous, and one-chambered. Galler: Lepidoptera (01 catterpilar). Path: Travessia Ruy Braga, 16/III/2015.

Previous gall records on this plant genus: in Atlantic forest: on Aureliana fasciculata (Vell.) Sendtn - Maia 2013b ( $\mathrm{n}=4 /$ Bertioga/SP), Maia 2001 ( $\mathrm{n}=1 /$ Carapebus/RJ), on A. glomultiflora Sendtn - Maia 2013a (n=1/Bertioga/SP).

Solanum asperum Rich. (native species, NE) $(n=1)$

Gall (Fig. 395): on leaf petiole and stem, globoid, yellowish, with trichomes, onechambered. Galler: not determined (empty galls). Path: Água Branca-Ruy Braga, 18/VI/2014.

No previous gall records on this plant.

Solanum incinellum Lindl (not found in Flora do Brasil, 2016) $(\mathrm{n}=1)$

Gall (Fig. 396): on leaf vein, fusiform, greenish, with trichomes, and one-chambered. Galler: Clinodiplosis sp. (Cecidomyiidae). Path: Três Picos, 13/V/2014, 08/IX/2014; Travessia Ruy Braga, 15/IX/2015. 
No previous gall records on this plant.

Solanum megalochiton Mart. (native species, NE) $(\mathrm{n}=1)$

Gall (Fig. 397): on leaf, globoid, green, pubescent, and one-chambered. Galler: Cecidomyiidae. Paths: Barbosa RodriguesMirante do Último Adeus, 09/XII/2015; BR-485, 09/XII/2014.

No previous gall records on this plant species. Solanum scuticum M.Ne (native species, NE) or piluliferum Dunal (endemic in Brazil, Atlantic forest, NE) $(\mathrm{n}=2)$

Gall (Fig. 398): on leaf, globoid, green, with trichomes, and one-chambered. Galler: Cecidomyiidae (04 larvae). Path: Casa 33, 16/ VII/2015.

Gall (Fig. 399): on leaf, discoid, green, and one-chambered. Galler: not determined. Path: Casa 33, 16/VII/2015.

No previous gall records on this plant species. Solanum swartzianum Roem. and Schult. (native species, NE) $(\mathrm{n}=1)$

Gall (Fig. 400): on leaf vein, fusiform, brown, woody, glabrous, and one-chambered. Galler: Cecidomyiidae (01 larva, 01 pupa). Paths: Hotel Donati, 04/VIII/2014; Travessia Ruy Braga, 1012/XI/2014, 16/III/2015, 17/III/2015; BR-485, 28/I/2015; Casa 33, 16/VI/2015.

Previous gall records on this plant species: in Atlantic forest - Maia et al. $2014(\mathrm{n}=1 /$ Santa Teresa/ES).

Solanum L. sp.1 (native genus) ( $\mathrm{n}=1$ )

Gall (Fig. 401): on stem, fusiform, green, glabrous, and one-chambered. Galler: Cecidomyiidae (02 larvae, 01 pupal exuvia, 02 adults). Parasitoids: Hymenoptera (03 adults). Paths: Cachoeira Véu da Noiva, 17/III/2014; Três Picos, 13/V/2014, 11/IX/2014; Viúva Hansen, 10/ IX/2014.

Solanum L. sp. 2 ( $\mathrm{n}=1)$

Gall (Fig. 402): on stem, leaf petiole and vein, fusiform, green, glabrous, and one-chambered
(Fig.). Galler: Asphondylia sp. (Cecidomyiidae) (01 young larva, 07 pupal exuviae, 08 females). Parasitoids: Hymenoptera (03 adults). Paths: Três Picos, 13/V/2014; Água Branca-Ruy Braga, 18/ VI/2014; Travessia Serra Negra, 14-15/X/2014.

Solanum L. sp.3 ( $\mathrm{n}=1)$

Gall (Fig. 403): on leaf vein, petiole, and stem, fusiform, green, micropubescent, and onechambered. Galler: Cecidomyiidae (01 pupal exuvia, 01 young larva). Parasitoids: Hymenoptera. Paths: Água Branca-Ruy Braga, 18/VI/2014; Travessia Ruy Braga, 08/VII/2014, 14/IV/2015, 15/IX/2015; Donati-Simon 2, 08/IX/2014.

Solanum L. sp.4 ( $\mathrm{n}=1)$

Gall (Fig. 404): on leaf vein, fusiform, yellowish, micropubescent, and one-chambered. Galler: not determined. Parasitoids: Hymenoptera (01 pupa). Path: Travessia Ruy Braga, 17/III/2015. Solanum L. sp.5 ( $\mathrm{n}=3)$

Gall (Fig. 405): on stem, fusiform, green, glabrous, and multichambered. Galler: not determined (empty galls). Path: Três Picos, 13/V/2014.

Gall (Fig. 406): on leaf petiole, fusiform, tubular or globoid, green or brownish, with white pubescence. Galler: Clinodiplosis sp. (Cecidomyiidae) (01 larva). Parasitoids: Hymenoptera (02 adults). Path: Três Picos, 11/ $\mathrm{XI} / 2014$.

Gall (Fig. 407): on leaf petiole and vein, fusiform, green, pubescent, and one-chambered. Galler: Cecidomyiidae (01 young larva). Paths: Três Picos, 13/V/2014: Água Branca-Ruy Braga, 18/VI/2014; Travessia Ruy Braga, 24/II/2015.

Solanum L. sp.6 $(\mathrm{n}=2)$

Gall (no fig.): on leaf, globoid, green, glabrous, and multichambered. Galler: Clinodiplosis sp. (Cecidomyiidae) (08 larvae). Paths: Travessia Ruy Braga, 18/VI/2014, Água Branca-Ruy Braga, 18/ $\mathrm{VI} / 2014$.

Gall (Fig. 408): on leaf, globoid, green, glabrous, and one-chambered. Galler: 


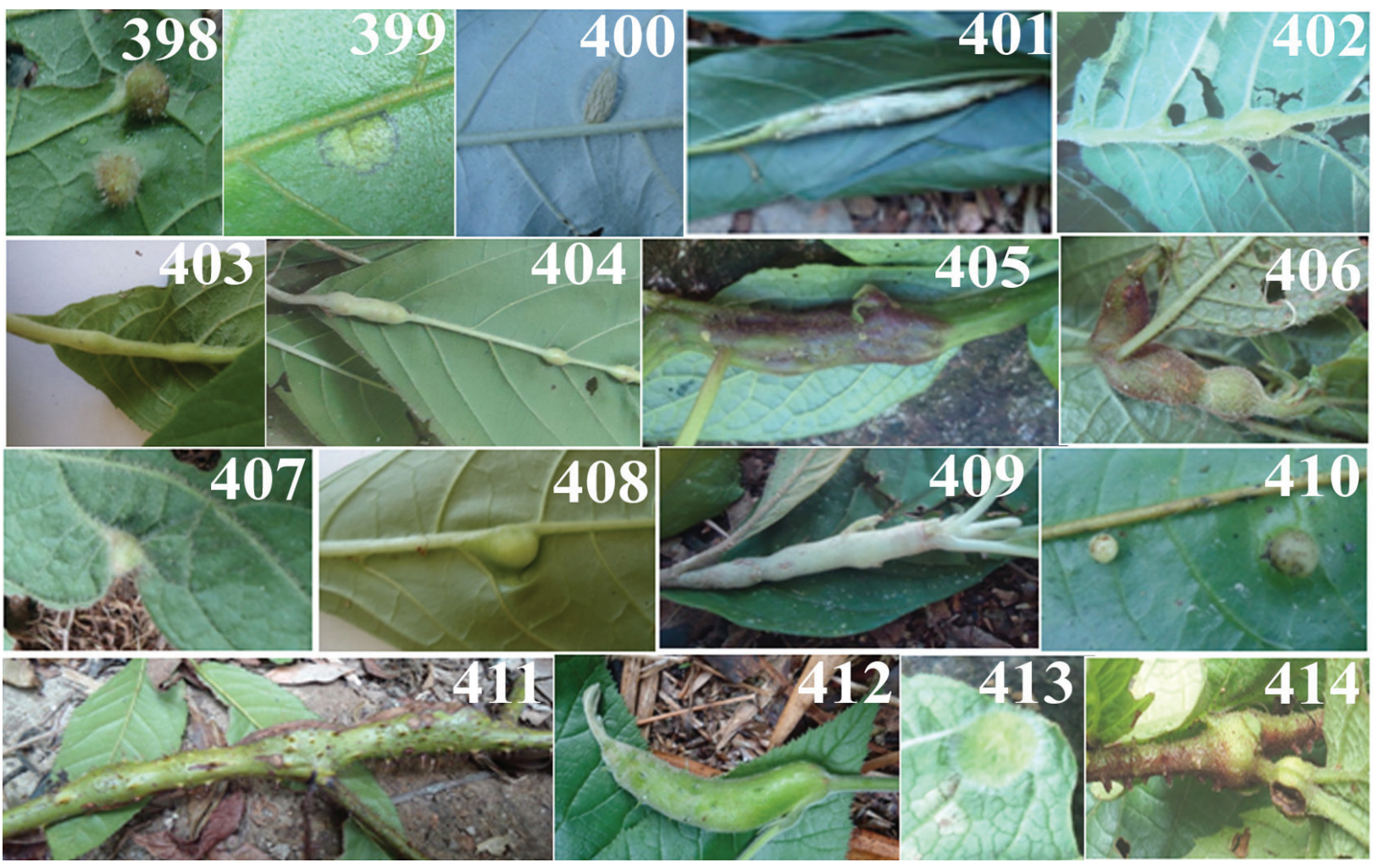

Figure 398-414 - Insect galls of the Parque Nacional do Itatiaia (Southeast Region, Brazil), 398-412, on Solanaceae, 398-399, Solanum scuticum or S. piluliferum, 398, globoid leaf gall, 399, discoid leaf gall, 400, S. swartzianum, fusiform leaf vein gall, 401, Solanum sp. 1, fusiform stem gall, 402, Solanum sp. 2, fusiform stem, leaf petiole and vein gall, 403, Solanum sp. 3, fusiform stem, leaf petiole and vein gall, 404, Solanum sp. 4, fusiform leaf vein gall, 405-407, Solanum sp. 5, 405, fusiform stem gall, 406, fusiform leaf petiole gall, 407, fusiform leaf petiole and vein gall, 408, Solanum sp. 6, globoid leaf gall, 409, Solanum sp. 7, fusiform stem gall, 410, Solanum sp. 8, globoid leaf gall, 411-412, Solanum sp. 9, 411, globoid or fusiform stem gall, 412, fusiform bud gall, 413, on Verbenaceae, Lantana camara, globoid leaf gall, 414, on Vitaceae, Cissus albida, globoid leaf petiole gall.

Cecidomyiidae (02 larvae). Paths: Água BrancaRuy Braga, 18/VI/2014; Travessia Ruy Braga, 18/ $\mathrm{VI} / 2014$.

Solanum L. sp.7 ( $\mathrm{n}=1)$

Gall (Fig. 409): on stem, fusiform, green or brown, with micropubescence, and one or multichambered. Galler: Muscomorpha (01 larva, 01 puparium). Paths: Água Branca-Ruy Braga, 18/ $\mathrm{VI} / 2014,10-12 / \mathrm{XI} / 2014$.

Solanum L. sp.8 $(\mathrm{n}=1)$

Gall(Fig.410): onleaf, globoid, green, glabrous, and one-chambered. Galler: Cecidomyiidae (01 larva). Parasitoids: Hymenoptera (01 larva). Path: Hotel Donati, 04/VIII/2014.

Solanum L. sp. $9(\mathrm{n}=2)$
Gall (Fig. 411): on stem, fusiform or globoid, green, thorny, and one-chambered. Galler: Cecidomyiidae (02 larvae). Paths: Travessia Ruy Braga, 14/IV/2015; Travessia Serra Negra, 14-15/ X/2014; BR-485, 23/II/2015.

Gall (Fig. 412): on bud, fusiform, green, thorny, and pubescent. Galler: not determined. Path: Travessia Ruy Braga, 10-12/XI/2014.

Previous gall records on undetermined species of Solanum L.: 1) in Atlantic forest - Fernandes and Negreiros 2006 ( $\mathrm{n}=1 /$ Aimorés//MG), Toma and Mendonça 2013 (n=1/ São Francisco de Paula/ RS), Maia 2013b (n=2/São Tomé das Letras/MG), Maia 2014 (n=1/Itamonte/MG), Maia et al. 2014 $(n=2 /$ Santa Teresa/ES), Rodrigues et al. $2014(n=2 /$ 
Mangaratiba/RJ), 2) in Amazonian forest - Maia 2011 (n=1/Oriximiná/PA), 3) in Cerrado - Araújo et al. $2015(\mathrm{n}=1 / \mathrm{GO})$.

\section{VERBENACEAE $(\mathrm{N}=1)$}

Lantana camara L. (native species, NE) ( $\mathrm{n}=1$ )

Gall (Fig. 413): on leaf, globoid, green, with trichomes, and one-chambered. Galler: Schismatodiplosis lantanae (01 larva) (Cecidomyiidae). Parasitoids: Hymenoptera (01 larva). Paths: Ecoarte-Lago Azul, 24/V/2014, L. S. Barbosa col.; Centro de Visitantes, 07-08/ VII/2014.

Previous records of the same gall morphotype: 1) in Atlantic forest - Rübsaamen 1908 (Cabo Frio/RJ and Tubarão/SC), Maia 2001 (Maricá/ RJ), Monteiro et al. 1994 (Maricá/RJ), Fernandes and Negreiros 2006 (Aimorés/MG), Proença and Maia 2014 (Rio das Ostras, Casimiro de Abreu, Valença/RJ), Rodrigues et al. 2014 (Mangaratiba/ RJ), 2) in Caatinga - Santos et al. 2001a (PE), 3) in Amazonian forest - Proença and Maia 2014 (Oriximiná/PA, Campo Novo de Rondônia/RO), 4) no data biomea - Mendonça et al. 2014 ( $n=1 / R S)$.

VITACEAE $(\mathrm{N}=1)$

Cissus albida Cambess. (endemic in Brazil, Caatinga, Cerrado and Atlantic forest, NE) $(n=1)$

Gall (Fig. 414): on leaf petiole, globoid, green, glabrous, and one-chambered. Galler: not determined (empty galls) Path: BR-485, EcoarteLago Azul, 28/I/2015.

No previous gall records on this plant species. Previous records on this plant genus: 1) in Pantanal: on C. spinosa - Julião et al. 2001 (n=1/MS), 2) in Atlantic forest: on Cissus sp. - Tavares 1918 ( $\mathrm{n}=1 /$ RJ), Rübsaamen 1908 ( $n=1 /$ RJ), 3) no biome data: on C. striata Ruiz and Pav. - Mendonça et al. 2014 $(\mathrm{n}=1 / \mathrm{RS})$.

\section{CONCLUSIONS}

Among all of the already surveyed areas of the Brazilian biomes, the PNI showed the greatest richness of insect galls. This park included 167 native host plants, being 63 endemic in Brazil, and two vulnerable species. The first hosted 365 gall morphotypes, the second 103 and the third three. The inducers of these galls were also proposed respectively as native, endemic and vulnerable. The gall richness of the lower part of the PNI was widely higher than that of the plateau. Asteraceae and Melastomataceae were the superhost families, as already pointed out in other Brazilian inventories. Our results confirmed the plant richness hypothesis and the plant architecture hypothesis, but they did not favor the harsh environment hypothesis.

Leaves were the most frequent galled plant organ and Cecidomyiidae (Diptera) were the most common galling taxon, as in the entire world. Most galls were globoid, green, glabrous and onechambered, which confirms the most frequent gall morphology in Brazil.

The associated fauna included parasitoids, inquilines and successors, the first were the most frequent, as in other Brazilian inventories. Predators were not found.

Several new records of host plants and gallers were found, contributing to the knowledge of their geographic distribution.

Our data highlight the PNI importance for the Atlantic forest biodiversity conservation.

\section{ACKNOWLEDGMENTS}

To the SISBio-ICMBio for license to research in the PNI unit (nr. 42434), to the PNI staff (General Director Gustavo W. Tomzhinski, Scientific Director Léo Nascimento and Coordinator of Public Use Leonardo Candido) for logistical support, to Dr. Gracialda C. Ferreira (Universidade Federal Rural do Amazonia, UFRAM, Pará) and Dr. Ricardo Moura (Jardim Botânico, RJ) for 
plant identification, and to Conselho Nacional de Desenvolvimento Científico e Tecnológico (CNPq) for financial support.

\section{REFERENCES}

ALMADA ED AND FERNANDES GW. 2011. Insetos indutores de galhas em florestas de terra firme e em reflorestamentos com espécies nativas na Amazônia Oriental, Pará, Brasil. Bol Mus Para Emílio Goeldi Cienc Nat 6: 163-196.

ARAÚJO WS, PORFÍRIO-JÚNIOR ED, JORGE VA AND ESPÍRITO-SANTO K. 2012. Plantas hospedeiras e galhas entomógenas em sub-bosques de florestas tropicais do Pará, Brasil. Insula Bol Horto Bot 41: 59-72.

ARAÚJO WS, PORFÍRIO-JÚNIOR ED, RIBEIRO BA, SILVA TM, SILVA EC, GUIMARÃES GFA, SCARELISANTOS C AND SANTOS BB. 2015. Checklist of host plants of insect galls in the state of Goiás in the Midwest Region of Brazil. Biodivers Data J 3: e6835.

ARAÚJO WS, SANTOS BB AND GOMES-KLEIN VL. 2011. Insect galls from Serra dos Pireneus, GO, Brazil. Biota Neotrop 11: 357-365.

ARAÚJO WS, SOBRAL FL AND MARACAHIPES L. 2014. Insect galls of the Parque Nacional das Emas (Mineiros, GO, Brazil). Check List 10: 1445-1451.

BREGONCI JM, POLYCARPO PV AND MAIA VC. 2010. Galhas de insetos do Parque Estadual Paulo César Vinha (Guarapari, ES, Brasil). Biota Neotrop 10: 265-274.

CARNEIRO MAA, BORGES RAX, ARAÚJO APA AND FERNANDES GW. 2009b. Insetos indutores de galhas da porção sul da Cadeia do Espinhaço, MG. Rev Bras Entomol 53: 570-592.

CARNEIRO MAA, BRANCO CSA, BRAGA CED, ALMADA ED, COSTA MBM AND MAIA VC. 2009a. Are gall midge species (Diptera, Cecidomyiidae) hostplant specialists? Rev Bras Entomol 53: 365-378.

CARVALHO-FERNANDES SP, ASCENDINO S, MAIA VC AND COURI MS. 2016. Diversity of insect galls associated with coastal shrub vegetation in Rio de Janeiro, Brazil. An Acad Bras Cienc 88: 1407-1418.

COELHO MS, ALMADA ED, FERNANDES GW, CARNEIRO MAA, SANTOS RM, QUINTINO AV AND SANCHEZ-AZOFEIFA A. 2009. Gall inducing arthropods from a seasonally dry tropical forest in Serra do Cipó, Brazil. Rev Bras Entomol 53: 404-414.

COELHO MS, CARNEIRO MAA, BRANCO C, BORGES RAX AND FERNANDES GW. 2013a. Gall-inducing insects from Campos de Altitude, Brazil. Biota Neotrop 13: $139-151$.

COELHO MS, CARNEIRO MAA, BRANCO C AND FERNANDES GW. 2013b. Gall-inducing insects from
Serra do Cabral, Minas Gerais, Brazil. Biota Neotrop 13: 102-109.

COSTA EC, CARVALHO-FERNANDES SP AND SANTOS-SILVA J. 2015. Galhas de insetos em uma área de transição Caatinga-Cerrado no Nordeste do Brasil. Sitientibus Ser Ci Biol 14: 10.13102/scb481

CUEVAS-REYES P, QUESADA M, HANSON P, DIRZO R AND OYAMA K. 2004. Diversity of gall-inducing insects in a Mexican tropical dry forest: the importance of plant species richness, life-forms, host plant age and plant density. J Ecol 92: 707-716.

FELT EP. 1940. Plant Galls and Gall Makers. Ithaca, New York, 364 p.

FERNANDES GW, ARAUJO RC, ARAUJO SC, LOMBARDI JA, PAULA AS, LOYOLA R AND CORNELISSEN TG. 1997. Insect galls from savana and rocky fields of the Jequitinhonha Valley, Minas Gerais, Brazil. Naturalia 22: 221-244.

FERNANDES GW, CARNEIRO MAA AND ISAIAS RMS. 2012. Gall-Inducing Insects: From Anatomy to Biodiversity. In: Panizzi AR and Parra JRP (Eds), Insect Bioecology and Nutrition for Integrated Pest Management (Boca Ratón, Florida, USA: CRC Press, Taylor \& Francis Group), p. 369-396.

FERNANDES GW, JULIÃO GR, ARAÚJO RC, ARAÚJO SC, LOMBARDI JA, NEGREIROS D AND CARNEIRO MA. 2001. Distribution and morphology of insect galls the Rio Doce Valley, Brazil. Naturalia 26: 211-244.

FERNANDES GW AND LARAACF. 1993. The Geography of galling insects and the mechanisms that result in patterns. In: PRICE PW, MATTSON WJ and BARANCHIKOV YN (Eds), The ecology and evolution of gall-forming insects. General Technical Report NC-174. United States Department of Agriculture, p. 42-47.

FERNANDES GW AND NEGREIROS D. 2006. A comunidade de insetos galhadores da RPPN Fazenda Bulcão, Aimorés, Minas Gerais, Brasil. Lundiana 7: 111-120.

FERNANDES GW AND PRICE PW. 1988. Biogeographical gradients in galling species richness: tests of hypotheses. Oecol 76: 161-167.

FERNANDES GW AND PRICE PW. 1992. The adaptative significance of insect gall distribution: survivorship of species in xeric and mesic habitats. Oecol 90: 14-20.

FERNANDES GW, TAMEIRÃO-NETO E AND MARTINS RP. 1988. Ocorrência e caracterização de galhas entomógenas na vegetação do Campus Pampulha da Universidade Federal de Minas Gerais. Rev Bras Zool 5: 11-29.

FLORA DO BRASIL. 2016. Disponível em: <http:// floradobrasil.jbrj.gov.br/>. Accessed in: 27.out.2016.

GAGNÉ RJ. 1977. The Cecidomyiidae associated with Chromolaena odorata (L.) K. and R (Compositae) in South America. Brenesia 12-13: 113-131. 
GAGNÉ RJ. 1994. The gall midges of the Neotropical region. Ithaca, Cornell University Press, 352 p.

GAGNÉ RJ, ODA RAM AND MONTEIRO RF. 2001. The gall midges (Diptera: Cecidomyiidae) of Mikania glomerata (Asteraceae) in Southeastern Brazil. Proc Entomol Soc Wash 103: 110-134.

GONÇALVES-ALVIM SJ AND FERNANDES GW. 2001. Comunidades de insetos galhadores (Insecta) em diferentes fisionomias do cerrado em Minas Gerais, Brasil. Rev Bras Zool 18: 289-305.

HOUARD C. 1933. Les Zoocécidies dês plantes del'Amérique du Sud et del'Amérique Central. Hermann et Cie, Paris, 519 p.

ICMBIO. 2016. Disponível em: <www.icmbio.gov.br/ parnaitatiaia $>$ Accessed in: 27.out.2016.

ISAIAS RMS, CARNEIRO RGS, OLIVEIRA DC AND SANTOS JC. 2013. Illustrated and Annotated Checklist of Brazilian Gall Morphotypes. Neotrop Entomol 42: 230239.

JARDIM BOTÂNICO DO RIO DE JANEIRO. 2016. Disponível em: <www.jbrj.gov/br/sites/all/themes/ corporateclean/content/publicacoes/plantas_floresta atlantica.pdf $>$ Accessed in: 27.out.2016.

JOY JB AND CRESPI BJ. 2007. Adaptive radiation of gall-inducing insects within a single host-plant species. Evolution 61: 784-795.

JULIÃO GR, AMARAL MEC AND FERNANDES GW. 2002. Galhas de insetos e suas plantas hospedeiras no pantanal Sul-Mato-Grossense. Naturalia 24: 47-74.

KIEFFER JJ. 1913. Diptera. Fam. Cecidomyidae. Fasc. 152, 346 p, 15 pls. In Wytsman, P., Ed. Genera Insectorum. Bruxelles.

KRAUS JE AND TANOE M. 1999. Morpho-ontogenetic aspects of entomogenous galls in roots of Cattleya guttata (Orchidaceae). Lindleyana 14: 204-213.

LARA ACF AND FERNANDES GW. 1996. The highest diversity of galling insects: Serra do Cipó, Brazil. Biodiv Letts 3: 111-114.

LARA ACF, FERNANDES GW AND GONÇALVESALVIM SJ. 2002. Tests of hypotheses on patterns of gall distribution along an altitudinal gradient. Trop Zool 15: 219-232.

LAWTON JH. 1983. Plant architecture and the diversity of phytophagous insects. Annu Rev Entomol 28: 23-29.

LIMA ES, MAGENTA MAG, KRAUS JE, VECHI C AND MARTINS SE. 2000. Levantamento preliminar de galhas entomógenas ocorrentes em plantas das restingas de Bertioga (SP), p. 39-46. Anais do V Simpósio de Ecossistemas Brasileiros: Conservação. III. ACIESP, 109 p.

LUZ GR, FERNANDES GW AND SILVA JO. 2012. Galhas de insetos em habitats xérico e mésico em região de transição Cerrado-Caatinga no norte de Minas Gerais, Brasil. Neotrop Biol Conserv 7: 171-187.
MAIA VC. 2001. The gall midges (Diptera, Cecidomyiidae) from three restingas of Rio de Janeiro State, Brazil. Rev Bras Zool 18: 305-656.

MAIA VC. 2006. Galls of Hemiptera, Lepidoptera and Thysanoptera from Central and South America. Publ Avul Mus Nac 110: 3-22.

MAIA VC. 2011. Characterization of insect galls, gall makers, and associated fauna of Platô Bacaba (Porto de Trombetas, Pará, Brazil). Biota Neotrop 11: 37-53.

MAIA VC. 2013a. Galhas de insetos em restingas da região sudeste do Brasil com novos registros. Biota Neotrop 13: 183-209.

MAIA VC. 2013b. Insect galls of São Tomé das Letras (MG, Brazil). Biota Neotrop 13: 164-189.

MAIA VC. 2014. Insect galls of Itamonte (Minas Gerais, Brazil): characterization and occurrence. Biota Neotrop 14: 1-17. http://www.biotaneotropica.org.br/v14n1/en/ abstract?article+bn01114012014

MAIA VC AND ARAÚJO LA. 2016. Clinodiplosis agerati (Diptera, Cecidomyiidae), a new galling species associated with Ageratum conyzoides (Asteraceae) from Brazil. Braz J Biol 7: 782-786.

MAIA VC, CARDOSO JLT AND BRAGA JMA. 2014. Insect galls from Atlantic Forest areas of Santa Teresa, Espírito Santo, Brazil: characterization and occurrence. Bol Mus Biol Mello Leitão (N. Sér.) 33: 47-129.

MAIA VC AND CARVALHO-FERNANDES SP. 2016. Insect galls of a protected remnant of the Atlantic Forest tableland from Rio de Janeiro State (Brazil) Rev Bras Entomol 60: 40-56.

MAIA VC AND FERNANDES GW. 2004. Insect Galls from Serra de São José (Tiradentes, MG, Brazil). Braz J Biol 64: 423-445.

MAIA VC, FERNANDES GW AND OLIVEIRA LA. 2010. A new species of Bruggmanniella (Diptera, Cecidomyiidae, Asphondyliini) associated with Doliocarpus dentatus (Dilleniaceae) in Brazil. Rev Bras Entomol 54: 225-228.

MAIA VC, MAGENTA MAG AND MARTINS SE. 2008. Ocorrência e caracterização de galhas de insetos em áreas de restinga de Bertioga (São Paulo, Brasil). Biota Neotrop 8: 167-197.

MAIA VC AND OLIVEIRA JC. 2010. Galhas de insetos da Reserva Biológica Estadual da Praia do Sul (Ilha Grande, Angra dos Reis, RJ). Biota Neotrop 10: 227-238.

MAIA VC AND SILVA LO. 2016. Insect galls of Restinga de Marambaia (Barra de Guaratiba, Rio de Janeiro, RJ). Braz J Biol 76: 787-795.

MAIA VC AND SOUZA MC. 2013. Insect galls of the xeric vegetation of Ilha do Cabo Frio (Arraial do Cabo, RJ, Brazil). Biota Neotrop 13: 278-288.

MALVES K AND FRIEIRO-COSTA FA. 2012. List of Plants with Galls Induced by Insects from the UNILAVRAS/ 
Boqueirão Biological Reserve, Ingaí, state of Minas Gerais, Brazil. Check List 8: 426-431.

MENDONÇA MS. 2001. Galling insect diversity: the resource synchronization hypothesis. Oikos 95: 171-176.

MENDONÇA MSJR, TOMA TSP AND SILVA JS. 2014. Galls and Galling Arthropods of Southern Brazil, p. 221256. In: Fernandes GW and Santos JC (Eds), Neotropical Insect Galls. Springer, London, $550 \mathrm{p}$.

MONTEIRO RF, FERRAZ FFF, MAIA VC AND AZEVEDO MAP. 1994. Galhas entomógenas em restingas: uma abordagem preliminar. An ACIESP 3(87): 210-220.

MONTEIRO RF, ODA RAM, NARAHARA KL AND CONSTANTINO PAL. 2004. Galhas: Diversidade, Especificidade e Distribuição, p.127-141. In: Rocha CFD, Esteves FA and Scarano (Eds), Pesquisa de Longa Duração na Restinga de Jurubatiba: Ecologia, História Natural e Conservação. RiMa Editora, São Carlos, 374 p.

OLIVEIRA JC AND MAIA VC. 2005. Ocorrência e caracterização de galhas de insetos na restinga de Grumari (Rio de Janeiro, RJ, Brasil). Arq Mus Nac 63(4): 669-675.

PRICE PW. 2005. Adaptive radiation of gall-inducing insects. Basic Appl. Ecol. 6: 413-421.

PRICE PW, FERNANDES GW, LARA ACF, BRAWN J, GERLING D, BARRIOS H, WRIGHT MG, RIBEIRO SP AND ROTHCLIFF N. 1998. Global patterns in local number of insect galling species. J Biogeogr 25: 581-591.

PROENÇA B AND MAIA VC. 2014. New state record of Schismatodiplosis lantanae (Rübsaamen, 1908) (Insecta, Diptera, Cecidomyiidae) in Brazil. Check List 10: 5571559.

RODRIGUES AR, MAIA VC AND COURI MS. 2014. Insect galls of restinga areas of Ilha da Marambaia, Rio de Janeiro, Brazil. Rev Bras Entomol 58: 173-197.

ROOT RB. 1973. Organization of a plant-arthropod association in simple and diverse habitats: the fauna of collards (Brassica oleraceae). Ecological Monographs 43: 95-124.

RÜBSAAMEN EH. 1905. Beiträge zur Kenntnis aussereuropäischer Zoocecidien. II. Beitrag: Gallen aus Brasilien und Peru. (Vorläufige Mitteilung). Marcellia 4: 65-85.

RÜBSAAMEN EH. 1908. Beiträge zur Kenntnis aussereuropäischer Zoocecidien. III. Beitrag: Gallen aus Brasilien und Peru. Marcellia 6: 110-173.

RÜBSAAMEN EH. 1916. Beitrag zur Kenntnis aussereuropäischer Gallmücken. S Ber Ges Naturf Fr Berl 1915: 431-481.

SAITO VS AND URSO-GUIMARÃES MV. 2012. Characterization of galls, insect galls and associated fauna of Ecological Station of Jataí (Luiz Antônio, SP.) Biota Neotrop 12: 99-107.
SÁIZ F AND NÚÑEZ C. 2000. Ecological aspects of galls in the arid north of Chile: second region. Rev Chilena Ent 26: 41-51.

SANTOS BB, FERREIRA HD AND ARAÚJO WS. 2010. Ocorrência e caracterização de galhas entomógenas em uma área de floresta estacional semidecídua em Goiânia, Goiás, Brasil. Acta Bot Bras 24: 243-249.

SANTOS JC, ALMEIDA-CORTEZ JS AND FERNANDES GW. 2011a. Richness of gall-inducing insects in the tropical dry forest (caatinga) of Pernambuco. Rev Bras Entomol 55: 45-54.

SANTOS JC, ALMEIDA-CORTEZ JS AND FERNANDES GW. 2011b. Diversity of gall-inducing insects in the high altitude wetland forests in Pernambuco, Northeastern Brazil. Braz J Biol 71: 47-56.

SANTOS JC, ALMEIDA-CORTEZ JS AND FERNANDES GW. 2012. Gall-inducing insects from Atlantic forest of Pernambuco, Northeastern Brazil. Biota Neotrop 12: 197213.

SOUTHWOOD TRE. 1960. The abundance of the Hawaiian trees and the number of their associated insect species. Proc Hawaii Entomol Soc 17: 299-303.

SOUTHWOOD TRE. 1961. The number of insect associated with various trees. J Anim Ecol 30: 1-8.

TAVARES JS. 1908. Contributio prima ad cognitionem cecidologiae regionis zambeziae (Moçambique, Africa Orientalis). Brotéria, Ser Zool 7: 133-171, pls. VII-XV.

TAVARES JS. 1909. Contributio prima ad cognitionem cecidologiae braziliae. Brotéria, Ser Zool 8: 5-28, pls. I-VIII.

TAVARES JS. 1916. Cecidomyias novas do Brazil. Brotéria, Ser Zool 14: 36-57.

TAVARES JS. 1917. Cecídias brazileiras que se criam em plantas das famílias das Compositae, Rubiaceae, Tiliaceae, Lythraceae e Artocarpaceae. Brotéria, Ser Zool 15: 113181, pls. VI-XI.

TAVARES JS. 1918. Cecidomyias novas do Brazil, segunda série. Brotéria, Ser Zool 16: 68-84, pls. III-IV.

TAVARES JS. 1920. [continuation of:] Cecidologia brazileira: cecídias que se criam em plantas das famílias das Leguminosae, Sapotaceae, Lauraceae, Myrtaceae, Punicaceae, Aurantiaceae, Malpighiaceae, Sapindaceae, Umbelliferae, Loranthaceae, Apocynaceae, Urticaceae, Salicaceae e Gramineae. Brotéria, Ser Zool 18: 97-125, pl. III.

TAVARES JS. 1922. Cecidologia brazileira: as restantes famílias. Brotéria, Ser Zool 19: 5-48, pls. XI-XIX.

TOMA TSP AND MENDONÇA MSJR. 2013. Gall-inducing insects of an Araucaria Forest in southern Brazil. Rev Bras Entomol 57: 225-233.

URSO-GUIMARÃES MV AND SCARELI-SANTOS C. 2006. Galls and gall makers in plants from the Pé-de- 
Gigante Cerrado Reserve, Santa Rita do Passa-Quatro SP, Brazil. Braz J Biol 66: 357-369.

URSO-GUIMARÃES MV, SCARELI-SANTOS C AND BONIFÁCIO-SILVA AC. 2003. Occurrence and Charaterization of entomogenous galls in plants from natural vegetation areas in Delfinópolis, $\mathrm{MG}$, Brazil. Braz J Biol 63: 705-715.
YUKAWA J AND ROHFRITSCH O. 2005. Biology and Ecology of Gall-inducing Cecidomyiidae (Diptera). In Biology, Ecology, and Evolution of Gall-inducing Arthropods, p. 273-304.

YUKAWA J, TOKUDA M, UECHI N AND SATO S. 2001. Species richness of galling arthropods in Manaus, Amazon and the surroundings of Iguassu Falls. Esakia 41: 11-15. 